\title{
EXISTENCE AND DECAY ESTIMATES FOR TIME DEPENDENT PARABOLIC EQUATION WITH APPLICATION TO DUNCAN-MORTENSEN-ZAKAI EQUATION*
}

\author{
SHING-TUNG YAU $^{\dagger}$ AND STEPHEN S.-T YAU ${ }^{\ddagger}$
}

Abstract. In this paper, we provide existence and estimates of the equation

$$
\frac{\partial u}{\partial t}=\Delta u+\sum_{i=1}^{n} f_{i} \frac{\partial u}{\partial x_{i}}-V u
$$

0. Introduction. In control theory or in many branches of applied mathematics, we are interested in an evolution equation of the following type:

$$
\frac{\partial u}{\partial t}=\Delta u+\sum_{i=1}^{n} f_{i} \frac{\partial u}{\partial x_{i}}-V u,
$$

where $f_{i}$ and $V$ are possibly time dependent functions.

Given an initial function at time zero, we would like to know existence of a positive solution of this equation. Furthermore if the initial function decay fast in spatial direction, we would like to know the spatial decay property of the solution for later time. In fact, in order for numerical calculation to be carried out effectively, we need to know quantitively this decay property. In this paper, we provide precise estimates of such an equation under reasonable assumptions on $f$ and $V$. In applications $f_{i}$ and $V$ may not be smooth in time. We have therefore avoided any argument involving differentiation of $f_{i}$ and $V$ in time. A typical equation that can be treated are those arised in nonlinear filtering problem where the robust Duncan-Mortensen-Zakai equation has our form. We demonstrate existence and give decay estimate of this equation.

D. Strook pointed out that his paper with Norris (Heat flows with uniformly elliptic coefficients, Proceedings LMS (3), Vol.62, \#2, (1991), 373-402) is closely related to section 1 of this paper where they treated the case with bounded coefficients. We were also informed that Fleming-Mitter, Sussmann, Baras-Blankenship-Hopkins have obtained important estimates on the DMZ equation. However the latter two papers are focused on one spatial dimension, while the former paper needs the boundedness of $f$ and $\nabla f$.

1. A priori estimations. To begin with, let us recall some well known formulas and inequality which will be used repeatedly throughout this paper.

Divergence Theorem Let $\Omega$ be a bounded domain with $C^{1}$-boundary $\partial \Omega$ and let $\nu$ denote the unit outward normal to $\partial \Omega$. For any vector field $w$ in $C^{0}(\bar{\Omega}) \cap C^{1}(\Omega)$,

$$
\int_{\Omega} \operatorname{div} w d x=\int_{\partial \Omega} w \cdot \nu d s
$$

\footnotetext{
*Received May 23, 1997; accepted for publication September 2, 1997.

${ }^{\dagger}$ Department of Mathematics, Harvard University, Cambridge, MA 02138, USA and Institute of Mathematical Sciences, Chinese University of Hong Kong, Shatin, Hong Kong (yau@math.harvard. edu). Research supported in part by ARO, NSF and IMS at the Chinese University of Hong Kong.

$\ddagger$ Control and Information Lab., MSCS, M/C 249, University of Illinois at Chicago, 851 S. Morgan St., Chicago, IL, 60607-7045 (yau@uic.edu). Research supported by ARO \# DAAH 04-1-0530 and NSF.
} 
where $d s$ denotes that $(n-1)$-dimensional area element in $\partial \Omega$.

More generally for any scalar valued function $\alpha \in C^{0}(\bar{\Omega}) \cap C^{1}(\Omega)$,

$$
\int_{\Omega} \alpha \operatorname{div} w d s=\int_{\partial \Omega} \alpha w \cdot \nu d s-\int_{\Omega} w \cdot \nabla \alpha d x
$$

Green's First Identity Let $\Omega$ be a domain for which the divergence theorem holds and let $w$ and $v$ be $C^{1}(\bar{\Omega}) \cap C^{2}(\Omega)$ functions, Then

$$
\int_{\Omega} v \Delta w d x=-\int_{\Omega} \nabla v \cdot \nabla w d x+\int_{\partial \Omega} v \frac{\partial w}{\partial \nu} d s .
$$

More generally for any $C^{1}(\bar{\Omega}) \cap C^{2}(\Omega)$ function $\alpha$,

$$
\int_{\Omega} \alpha \nabla v \cdot \nabla w d x=-\int_{\Omega} \alpha v \Delta w d x-\int_{\Omega} v \nabla \alpha \cdot \nabla w d x+\int_{\partial \Omega} \alpha v \frac{\partial w}{\partial \nu} d s .
$$

The following inequality is true for any $a, b \in \mathbb{R}$ and any $\varepsilon>0$,

$$
a b \leq \varepsilon a^{2}+\frac{1}{4 \varepsilon} b^{2} .
$$

For arbitrary real numbers $a_{1}, a_{2}, \cdots, a_{n}$, we have

$$
\left(a_{1}+\cdots+a_{n}\right)^{2} \leq n a_{1}^{2}+n a_{2}^{2}+\cdots+n a_{n}^{2} .
$$

We are now ready to prove the first theroem in this section.

THEOREM 1.1. Let $\Omega$ be a compact domain in $\mathbb{R}^{n}$ with $C^{1}$-boundary $\partial \Omega$ and let $\nu$ denote the unit outward normal to $\partial \Omega$. Let $f_{1}(x, t), \cdots, f_{n}(x, t)$ and $V(x, t)$ be smooth functions in $x$-variable. Suppose that $f_{1}, \cdots, f_{n}$ vanish on $\partial \Omega$. Let $u$ be a solution of the equation

$$
\frac{\partial u}{\partial t}=\Delta u+\sum_{i=1}^{n} f_{i} \frac{\partial u}{\partial x_{i}}-V u
$$

with boundary condition $\frac{\partial u}{\partial \nu}=0$ on $\partial \Omega$.

(i) If $\frac{\partial g}{\partial t}+\frac{1}{2}|\nabla g|^{2}-\sum_{i=1}^{n} f_{i} \frac{\partial g}{\partial x_{i}}-\operatorname{div} f-2 V \leq 0$, for $0 \leq t \leq T$, then

$$
\int_{\Omega \times\{T\}} e^{g} u^{2} \leq \int_{\Omega \times\{0\}} e^{g} u^{2}
$$

(ii) If $\frac{\partial h}{\partial t}+|\nabla h+f|^{2}-2 V+|\nabla V|^{2} \leq 0$, for $0 \leq t \leq T$, then

$$
\int_{\Omega \times\{T\}} e^{h}|\nabla u|^{2} \leq \int_{\Omega \times\{0\}} e^{h}|\nabla u|^{2}+\int_{0}^{T} \int_{\Omega} e^{h} u^{2} .
$$

(iii) If $\frac{\partial \rho}{\partial t}+\left(\varepsilon_{1}+\frac{1}{2 \varepsilon_{1}^{2}}\right)|\nabla \rho|^{2}+\sum_{i=1}^{n} \rho_{i} f_{i}+\sum_{i=1}^{n} f_{i, i} \leq 0$ for $0 \leq t \leq T$, where $2-10 \varepsilon_{1}^{2}-\frac{2 \varepsilon_{1}}{1-3 \varepsilon_{1}}>0$, then

(1.4) $\int_{\Omega \times\{T\}} e^{\rho}(\Delta u)^{2} \leq \int_{\Omega \times\{0\}} e^{\rho}(\Delta u)^{2}+O\left(\int_{\Omega \times[0, T]} e^{\rho}|\nabla \rho|^{2}|f|^{2}|\nabla u|^{2}\right.$ 


$$
\begin{aligned}
& +\int_{\Omega \times[0, T]} e^{\rho}|\nabla f|^{2}|\nabla u|^{2}+\int_{\Omega \times[0, T]} e^{\rho}|f||\nabla u|^{2}|\triangle f|+\int_{\Omega \times[0, T]} e^{\rho}|f|^{4}|\nabla u|^{2} \\
& \left.+\int_{\Omega \times[0, T]} e^{\rho}|\nabla(V u)|^{2}+\int_{\Omega \times[0, T]} e^{\rho}|\nabla u|^{2}\left(\sum_{i=1}^{n} f_{i, i}\right)^{2}\right)
\end{aligned}
$$

Proof. (i) Equation (1.1) implies

$$
\int_{\Omega} \int_{0}^{T} e^{g} u\left(\frac{\partial u}{\partial t}-\Delta u-\sum_{i=1}^{n} f_{i} \frac{\partial u}{\partial x_{i}}+V u\right)=0 .
$$

Integrating by parts, we have

$$
\begin{aligned}
0 & =\int_{\Omega \times\{T\}} \frac{e^{g} u^{2}}{2}-\int_{\Omega \times\{0\}} \frac{e^{g} u^{2}}{2}-\int_{\Omega} \int_{0}^{T} e^{g} \frac{u^{2}}{2} \frac{\partial g}{\partial t} \\
& +\int_{\Omega} \int_{0}^{T} e^{g} u \nabla g \cdot \nabla u+\int_{\Omega} \int_{0}^{T} e^{g}|\nabla u|^{2} \\
& +\int_{\Omega} \int_{0}^{T} e^{g} \frac{u^{2}}{2} \sum_{i=1}^{n} f_{i} g_{i}+\int_{\Omega} \int_{0}^{T} e^{g} \frac{u^{2}}{2} \sum_{i=1}^{n} f_{i, i} \\
& +\int_{\Omega} \int_{0}^{T} e^{g} u^{2} V
\end{aligned}
$$

where $g_{i}$ denotes $\frac{\partial g}{\partial x_{i}}$ and $f_{i, i}$ denotes $\frac{\partial f_{i}}{\partial x_{i}}$. The boundary condition for (1.3)

$$
\int_{\partial \Omega} \int_{0}^{T} u\left(\frac{\partial u}{\partial \nu}+\frac{1}{2} u \sum_{i=1}^{n} f_{i} \nu_{i}\right)
$$

vanishes because $\frac{\partial u}{\partial \nu}, f_{1} \cdots, f_{n}$ vanish on $\partial \Omega$. But

$$
\left|\int_{\Omega} \int_{0}^{T} e^{g} u \nabla g \cdot \nabla u\right| \leq \frac{1}{4} \int_{\Omega} \int_{0}^{T} e^{g} u^{2}|\nabla g|^{2}+\int_{\Omega} \int_{0}^{T} e^{g}|\nabla u|^{2} .
$$

Put the above inequality in (1.3), we get

$$
\begin{aligned}
& \int_{\Omega \times\{T\}} e^{g} \frac{u^{2}}{2} \\
\leq & \int_{\Omega \times\{0\}} e^{g} \frac{u^{2}}{2}+\int_{\Omega} \int_{0}^{T} e^{g} \frac{u^{2}}{2} \frac{\partial g}{\partial t}+\frac{1}{4} \int_{\Omega} \int_{0}^{T} e^{g} u^{2}|\nabla g|^{2} \\
& +\int_{\Omega} \int_{0}^{T} e^{g}|\nabla u|^{2}-\int_{\Omega} \int_{0}^{T} e^{g}|\nabla u|^{2}-\int_{\Omega} \int_{0}^{T} e^{g} \frac{u^{2}}{2} \sum_{i=1}^{n} f_{i} \frac{\partial g}{\partial x_{i}} \\
& -\int_{\Omega} \int_{0}^{T} e^{g} \frac{u^{2}}{2} \sum_{i=1}^{n} f_{i, i}-\int_{\Omega} \int_{0}^{T} e^{g} V u^{2} \\
= & \int_{\Omega \times\{0\}} e^{g} \frac{u^{2}}{2}+\int_{\Omega} \int_{0}^{T} e^{g} \frac{u^{2}}{2}\left[\frac{\partial g}{\partial t}+\frac{1}{2}|\nabla g|^{2}-\sum_{i=1}^{n} f_{i} \frac{\partial g}{\partial x_{i}}-\sum_{i=1}^{n} f_{i, i}-2 V\right] .
\end{aligned}
$$


Hence if

$$
\frac{\partial g}{\partial t}-\frac{1}{2}|\nabla g|^{2}-\sum_{i=1}^{n} f_{i} \frac{\partial g}{\partial x_{i}}-\sum_{i=1}^{n} f_{i, i}-2 V \leq 0 \text { on } \Omega \times[0, T]
$$

then we have

$$
\int_{\Omega \times\{T\}} \frac{e^{g} u^{2}}{2} \leq \int_{\Omega \times\{0\}} \frac{e^{g} u^{2}}{2} .
$$

(ii) By a similar argument, we obtain

$$
\begin{aligned}
& \int_{\Omega \times\{T\}} e^{h}|\nabla u|^{2}-\int_{\Omega \times\{0\}} e^{h}|\nabla u|^{2}=\int_{\Omega \times[0, T]} \frac{\partial}{\partial t}\left(e^{h}|\nabla u|^{2}\right) \\
= & \int_{\Omega \times[0, T]} e^{h} \frac{\partial h}{\partial t}|\nabla u|^{2}+2 \int_{\Omega \times[0, T]} e^{h} \nabla u \cdot \nabla u_{t} \\
= & \int_{\Omega \times[0, T]} e^{h} h_{t}|\nabla u|^{2}-2 \int_{\Omega \times[0, T]} e^{h}(\nabla u \cdot \nabla h) u_{t}-2 \int_{\Omega \times[0, T]} e^{h}(\Delta u) u_{t}
\end{aligned}
$$

because of the vanishing of the boundary condition $u_{t} \frac{\partial u}{\partial \nu}$ on $\partial \Omega \times[0, T]$. So we have

$$
\begin{aligned}
& \int_{\Omega \times\{T\}} e^{h}|\nabla u|^{2}-\int_{\Omega \times\{0\}} e^{h}|\nabla u|^{2}=\int_{\Omega \times[0, T]} e^{h} h_{t}|\nabla u|^{2} \\
- & 2 \int_{\Omega \times[0, T]} e^{h}(\nabla u \cdot \nabla h)\left[\Delta u+\sum_{j=1}^{n} f_{j} u_{j}-V u\right] \\
- & 2 \int_{\Omega \times[0, T]} e^{h}(\Delta u)\left[\Delta u+\sum_{j=1}^{n} f_{j} u_{j}-V u\right] .
\end{aligned}
$$

But

$$
\begin{aligned}
& 2 \int_{\Omega \times[0, T]} e^{h}(\Delta u) V u=-2 \int_{\Omega \times[0, T]} e^{h}(\nabla u \cdot \nabla h) V u \\
& -2 \int_{\Omega \times[0, T]} e^{h}\left[u \nabla u \cdot \nabla V+V|\nabla u|^{2}\right]
\end{aligned}
$$

because of the vanishing of the boundary condition $V u \frac{\partial u}{\partial \nu}$ on $\partial \Omega \times[0, T]$.

Equations (1.8) and (1.9) imply

$$
\begin{aligned}
& \int_{\Omega \times\{T\}} e^{h}|\nabla u|^{2}-\int_{\Omega \times\{0\}} e^{h}|\nabla u|^{2} \\
= & \int_{\Omega \times[0, T]} e^{h} h_{t}|\nabla u|^{2}-2 \int_{\Omega \times[0, T]} e^{h}(\nabla u \cdot \nabla h)\left[\Delta u+\sum_{j=1}^{n} f_{j} u_{j}\right] \\
& -2 \int_{\Omega \times[0, T]} e^{h}(\Delta u)^{2}-2 \int_{\Omega \times[0, T]} e^{h}(\Delta u)\left(\sum_{j=1}^{n} f_{j} u_{j}\right)
\end{aligned}
$$




$$
-2 \int_{\Omega \times[0, T]} e^{h} u \nabla u \cdot \nabla V-2 \int_{\Omega \times[0, T]} e^{h} V|\nabla u|^{2} .
$$

By choosing coordinates, we can assume $u_{i}=0$ for $i>1$. Then

$$
\begin{aligned}
& 2((\nabla h+f) \cdot \nabla u) \Delta u+2(\nabla u \cdot \nabla h)\left(\sum_{i=1}^{n} f_{i} u_{i}\right) \\
= & 2\left(h_{1}+f_{1}\right) u_{1} \Delta u+2 u_{1}^{2} f_{1} h_{1} \\
\leq & h_{1}^{2} u_{1}^{2}+(\triangle u)^{2}+f_{1}^{2} u_{1}^{2}+(\triangle u)^{2}+2 u_{1}^{2} f_{1} h_{1} \\
= & \left(h_{1}+f_{1}\right)^{2} u_{1}^{2}+2(\Delta u)^{2} \\
\leq & |\nabla h+f|^{2}|\nabla u|^{2}+2(\triangle u)^{2} .
\end{aligned}
$$

Equations (1.10) and (1.11) imply

$$
\begin{aligned}
& \int_{\Omega \times\{T\}} e^{h}|\nabla u|^{2}-\int_{\Omega \times\{0\}} e^{h}|\nabla u|^{2} \\
= & \int_{\Omega \times[0, T]} e^{h} h_{t}|\nabla u|^{2}-2 \int_{\Omega \times[0, T]} e^{h}[(\nabla h+f) \cdot \nabla u] \Delta u \\
& -2 \int_{\Omega \times[0, T]} e^{h}(\nabla u \cdot \nabla h) \sum_{j=1}^{n} f_{j} u_{j}-2 \int_{\Omega \times[0, T]} e^{h}(\Delta u)^{2} \\
& -2 \int_{\Omega \times[0, T]} e^{h} u \nabla u \cdot \nabla V-2 \int_{\Omega \times[0, T]} e^{h} V|\nabla u|^{2} \\
\leq & \int_{\Omega \times[0, T]} e^{h} h_{t}|\nabla u|^{2}+\int_{\Omega \times[0, T]}\left(e^{h}|\nabla h+f|^{2}|\nabla u|^{2}+2 e^{h}(\Delta u)^{2}\right) \\
& -2 \int_{\Omega \times[0, T]} V e^{h}|\nabla u|^{2}-2 \int_{\Omega \times[0, T]} e^{h}(\Delta u)^{2}-2 \int_{\Omega \times[0, T]} e^{h} u \nabla u \cdot \nabla V \\
\leq & \int_{\Omega \times[0, T]} e^{h}|\nabla u|^{2}\left(h_{t}+|\nabla h+f|^{2}-2 V\right)+\int_{\Omega \times[0, T]} e^{h}\left(u^{2}+|\nabla u|^{2}|\nabla V|^{2}\right) \\
= & \int_{\Omega \times[0, T]} e^{h}|\nabla u|^{2}\left(h_{t}+|\nabla h+f|^{2}-2 V+|\nabla V|^{2}+\int_{\Omega \times[0, T]} e^{h} u^{2} .\right.
\end{aligned}
$$

Since

$$
h_{t}+|\nabla h+f|^{2}-2 V+|\nabla V|^{2} \leq 0, \quad \text { on } \Omega \times[0, T]
$$

by assumption, we see that

$$
\int_{\Omega \times\{T\}} e^{h}|\nabla u|^{2} \leq \int_{\Omega \times\{0\}} e^{h}|\nabla u|^{2}+\int_{0}^{T} \int_{\Omega} e^{h} u^{2} .
$$

(iii) Similary we can estimate the higher order derivatives of $u$ in the following way

$$
\begin{aligned}
& \int_{\Omega \times\{T\}} e^{\rho}(\Delta u)^{2}-\int_{\Omega \times\{0\}} e^{\rho}(\Delta u)^{2} \\
= & \int_{\Omega \times[0, T]} \frac{d}{d t}\left[e^{\rho}(\Delta u)^{2}\right]
\end{aligned}
$$




$$
\begin{aligned}
= & \int_{\Omega \times[0, T]} e^{\rho} \rho_{t}(\Delta u)^{2}+2 \int_{\Omega \times[0, T]} e^{\rho}(\Delta u) \Delta u_{t} \\
= & \int_{\Omega \times[0, T]} e^{\rho} \rho_{t}(\Delta u)^{2}-2 \int_{\Omega \times[0, T]} e^{\rho} \Delta u \nabla \rho \cdot \nabla\left(\Delta u+\sum_{i=1}^{n} f_{i} u_{i}-V u\right) \\
& -2 \int_{\Omega \times[0, T]} e^{\rho} \nabla(\Delta u) \cdot \nabla\left(\Delta u+\sum_{i=1}^{n} f_{i} u_{i}-V u\right)
\end{aligned}
$$

because of the vanishing of the boundary condition $\Delta u \frac{\partial u_{t}}{\partial \nu}$ on $\partial \Omega \times[0, T]$. Hence

$$
\begin{aligned}
& \int_{\Omega \times\{T\}} e^{\rho}(\Delta u)^{2}-\int_{\Omega \times\{0\}} e^{\rho}(\Delta u)^{2} \\
\leq & \int_{\Omega \times[0, T]} e^{\rho} \rho_{t}(\Delta u)^{2}-2 \int_{\Omega \times[0, T]} e^{\rho}(\Delta u) \nabla \rho \\
& +\frac{\varepsilon}{2} \int_{\Omega \times[0, T]} e^{\rho}(\Delta u)^{2}|\nabla \rho|^{2}+\frac{2}{\varepsilon} \int_{\Omega \times[0, T]} e^{\rho}\left|\nabla\left(\sum_{i=1}^{n} f_{i} u_{i}\right)\right|^{2} \\
& +\frac{\varepsilon}{2} \int_{\Omega \times[0, T]} e^{\rho}(\Delta u)^{2}|\nabla \rho|^{2}+\frac{2}{\varepsilon} \int_{\Omega \times[0, T]} e^{\rho}|\nabla(V u)|^{2} \\
& -2 \int_{\Omega \times[0, T]} e^{\rho}|\nabla(\Delta u)|^{2}-2 \int_{\Omega \times[0, T]} e^{\rho} \nabla(\Delta u) \cdot \nabla\left(\sum_{i=1}^{n} f_{i} u_{i}\right) \\
& +2 \int_{\Omega \times[0, T]} e^{\rho} \nabla(\Delta u) \cdot \nabla(V u) \\
= & \int_{\Omega \times[0, T]} e^{\rho} \rho_{t}(\Delta u)^{2}-2 \int_{\Omega \times[0, T]} e^{\rho} \Delta u \nabla \rho \cdot \nabla(\Delta u) \\
& +\varepsilon \int_{\Omega \times[0, T]} e^{\rho}(\Delta u)^{2}|\nabla \rho|^{2}+\frac{2}{\varepsilon} \int_{\Omega \times[0, T]} e^{\rho}\left|\nabla\left(\sum_{i=1}^{n} f_{i} u_{i}\right)\right|^{2} \\
& +\frac{2}{\varepsilon} \int_{\Omega \times[0, T]} e^{\rho}|\nabla(V u)|^{2}-2 \int_{\Omega \times[0, T]} e^{\rho}|\nabla(\Delta u)|^{2} \\
& -2 \int_{\Omega \times[0, T]} e^{\rho} \nabla(\Delta u) \cdot \nabla\left(\sum_{i=1}^{n} f_{i} u_{i}\right)+2 \int_{\Omega \times[0, T]} e^{\rho} \nabla(\Delta u) \cdot \nabla(V u) .
\end{aligned}
$$

But

$$
\begin{aligned}
& -2 \int_{\Omega \times[0, T]} e^{\rho} \nabla(\Delta u) \cdot \nabla\left(\sum_{i=1}^{n} f_{i} u_{i}\right) \\
= & -2 \int_{\Omega \times[0, T]} e^{\rho} \sum_{i, k=1}^{n}(\Delta u)_{k} u_{i} f_{i, k}-2 \int_{\Omega \times[0, T]} e^{\rho} \sum_{i, k=1}^{n}(\Delta u)_{k} f_{i} u_{i k} \\
& \quad\left(\text { where } f_{i, k}=\frac{\partial f_{i}}{\partial x_{k}}\right) \\
= & -2 \int_{\Omega \times[0, T]} e^{\rho} \sum_{k=1}^{n}(\Delta u)_{k} u_{i} f_{i, k}+2 \int_{\Omega \times[0, T]} e^{\rho} \sum_{i, k=1}^{n} \rho_{i}(\Delta u)_{k} u_{k} f_{i}
\end{aligned}
$$




$$
+2 \int_{\Omega \times[0, T]} e^{\rho} \sum_{i, k=1}^{n}(\Delta u)_{k i} f_{i} u_{k}+2 \int_{\Omega \times[0, T]} e^{\rho} \sum_{i, k=1}^{n}(\Delta u)_{k} u_{k} f_{i, i}
$$

because of the vanishing of the boundary conditions $u_{k}(\Delta u)_{k} f \cdot \nu, 1 \leq k \leq n$, on $\partial \Omega \times[0, T]$. Observe that

$$
\begin{aligned}
& 2 \int_{\Omega \times[0, T]} e^{\rho} \sum_{i, k=1}^{n}(\Delta u)_{k i} f_{i} u_{k}=2 \sum_{i=1}^{n} \int_{\Omega \times[0, T]} e^{\rho} f_{i}\left(\nabla(\Delta u)_{i} \cdot \nabla u\right) \\
= & -2 \sum_{i=1}^{n} \int_{\Omega \times[0, T]} e^{\rho} f_{i}(\Delta u)_{i} \nabla \rho \cdot \nabla u-2 \sum_{i=1}^{n} \int_{\Omega \times[0, T]} e^{\rho}(\Delta u)_{i} \nabla f_{i} \cdot \nabla u \\
& -2 \sum_{i=1}^{n} \int_{\Omega \times[0, T]} e^{\rho} f_{i}(\Delta u)_{i} \Delta u
\end{aligned}
$$

because of the vanishing of the boundary conditions $f_{i}(\Delta u)_{i} \frac{\partial u}{\partial \nu}, 1 \leq i \leq n$, on $\partial \Omega \times[0, T]$. Now the last terms in (1.17) is

$$
\begin{aligned}
& \left.-2 \int_{\Omega \times[0, T]}\left(e^{\rho} \Delta u\right) f \cdot \nabla(\Delta u)=2 \int_{\Omega \times[0, T]} \Delta u\right) \operatorname{div}\left[e^{\rho}(\triangle u) f\right] \\
& \text { (since }(\triangle u)^{2} f \cdot \nu=0 \text { on } \partial \Omega \times[0, T] \text { ) } \\
& =2 \int_{\Omega \times[0, T]}(\triangle u) \nabla\left(e^{\rho} \Delta u\right) \cdot f+2 \int_{\Omega \times[0, T]}(\triangle u) e^{\rho}(\Delta u)(\operatorname{div} f) \\
& =2 \int_{\Omega \times[0, T]}(\Delta u)^{2} e^{\rho} \nabla \rho \cdot f+2 \int_{\Omega \times[0, T]}(\Delta u) e^{\rho} \nabla(\Delta u) \cdot f \\
& +2 \int_{\Omega \times[0, T]}(\triangle u) e^{\rho}(\triangle u)(\operatorname{div} f) .
\end{aligned}
$$

The above equation implies

$$
(1.17)-2 \int_{\Omega \times[0, T]}\left(e^{\rho} \Delta u\right) f \cdot \nabla(\triangle u)=\int_{\Omega \times[0, T]}(\Delta u)^{2} e^{\rho} \nabla \rho \cdot f+\int_{\Omega \times[0, T]}(\Delta u)^{2} e^{\rho}(\operatorname{div} f) .
$$

Putting (1.18) into (1.17), we get

$$
\begin{aligned}
& 2 \int_{\Omega \times[0, T]} e^{\rho} \cdot \sum_{i, k=1}^{n}(\Delta u)_{k i} f_{i} u_{k} \\
= & -2 \int_{\Omega \times[0, T]} \sum_{i=1}^{n} e^{\rho} f_{i}(\Delta u)_{i} \nabla \rho \cdot \nabla u-2 \sum_{i=1}^{n} \int_{\Omega \times[0, T]} e^{\rho}(\Delta u)_{i} \nabla f_{i} \cdot \nabla u \\
& +\int_{\Omega \times[0, T]}(\Delta u)^{2} e^{\rho} \nabla \rho \cdot f+\int_{\Omega \times[0, T]}(\Delta u)^{2} \rho^{2} \operatorname{div} f .
\end{aligned}
$$

In view of (1.19), (1.16) becomes

$$
-2 \int_{\Omega \times[0, T]} e^{\rho} \nabla(\triangle u) \cdot \nabla\left(\sum_{i=1}^{n} f_{i} u_{i}\right)
$$




$$
\begin{aligned}
= & -2 \int_{\Omega \times[0, T]} e^{\rho} \sum_{k=1}^{n}(\Delta u)_{k} u_{i} f_{i, k}+2 \int_{\Omega \times[0, T]} \cdot e^{\rho} \sum_{i, k=1}^{n} \rho_{i}(\Delta u)_{k} u_{k} f_{i} \\
& +2 \int_{\Omega \times[0, T]} e^{\rho} \sum_{i, k=1}^{n}(\Delta u)_{k} u_{k} f_{i, i}-2 \int_{\Omega \times[0, T]} \sum_{i, k=1}^{n} e^{\rho} \rho_{k}(\Delta u)_{i} f_{i} u_{k} \\
& -2 \int_{\Omega \times[0, T]} \sum_{i, k=1}^{n} e^{\rho}(\Delta u)_{i} u_{k} f_{i, k}+\int_{\Omega \times[0, T]} \sum_{i=1}^{n} \rho_{i} f_{i}(\Delta u)^{2} \\
& +\int_{\Omega \times[0, T]} e^{\rho}\left(\sum_{i=1}^{n} f_{i, i}\right)(\Delta u)^{2} \\
= & -2 \int_{\Omega \times[0, T]} \sum_{k=1}^{n} e^{\rho}(\Delta u)_{k}\left[\sum_{i=1}^{n} u_{i}\left(f_{i, k}+f_{k, i}\right)-u_{k} \sum_{i=1}^{n} f_{i} \rho_{i}+f_{k} \sum_{i=1}^{n} u_{i} \rho_{i}\right. \\
& \left.-u_{k} \sum_{i=1}^{n} f_{i, i}\right]+\int_{\Omega \times[0, T]}^{n} e^{\rho} \sum_{i=1}^{n} \rho_{i} f_{i}(\Delta u)^{2}+\int_{\Omega \times[0, T]} e^{\rho}\left(\sum_{i=1}^{n} f_{i, i}\right)(\Delta u)^{2} .
\end{aligned}
$$

We next look at

$$
\begin{aligned}
& \int_{\Omega \times[0, T]} e^{\rho}\left|\nabla \sum_{i=1}^{n} f_{i} u_{i}\right|^{2} \\
& =-\int_{\Omega \times[0, T]} e^{\rho} \sum_{k=1}^{n} \rho_{k}\left(\sum_{i=1}^{n} f_{i} u_{i}\right)_{k}\left(\sum_{j=1}^{n} f_{j} u_{j}\right) \\
& -\int_{\Omega \times[0, T]} \sum_{k=1}^{n} e^{\rho}\left(\sum_{i=1}^{n} f_{i, k} u_{i}+\sum_{i=1}^{n} f_{i} u_{i, k}\right)_{k}\left(\sum_{j=1}^{n} f_{j} u_{j}\right) \\
& \leq \varepsilon \int_{\Omega \times[0, T]} e^{\rho}\left|\nabla \sum_{j=1}^{n} f_{j} u_{j}\right|^{2}+\frac{1}{4 \varepsilon} \int_{\Omega \times[0, T]}^{n} e^{\rho}|\nabla \rho|^{2}\left(\sum_{j=1}^{n} f_{j} u_{j}\right)^{2} \\
& -\int_{\Omega \times[0, T]} \sum_{k=1}^{n} e^{\rho}\left(\sum_{i=1}^{n} f_{i, k} u_{i}\right)_{k}\left(\sum_{j=1}^{n} f_{j} u_{j}\right)-\int_{\Omega \times[0, T]} \sum_{k=1}^{n} e^{\rho}\left(\sum_{i=1}^{n} f_{i} u_{i, k}\right)_{k}\left(\sum_{j=1}^{n} f_{j} u_{j}\right)
\end{aligned}
$$$$
=\varepsilon \int_{\Omega \times[0, T]} e^{\rho}\left|\nabla \sum_{j=1}^{n} f_{j} u_{j}\right|^{2}+\frac{1}{4 \varepsilon} \int_{\Omega \times[0, T]} e^{\rho}|\nabla \rho|^{2}\left(\sum_{j=1}^{n} f_{j} u_{j}\right)^{2}
$$$$
+\int_{\Omega \times[0, T]} e^{\rho}\left(\sum_{k=1}^{n} \sum_{i=1}^{n} \rho_{k} f_{i, k} u_{i}\right)\left(\sum_{j=1}^{n} f_{j} u_{j}\right)+\int_{\Omega \times[0, T]} e^{\rho} \sum_{k=1}^{n}\left(\sum_{i=1}^{n} f_{i, k} u_{i}\right)\left(\sum_{j=1}^{n} f_{j} u_{j}\right)_{k}
$$

$$
\begin{gathered}
-\int_{\Omega \times[0, T]} e^{\rho}\left(\sum_{k=1}^{n} \sum_{i=1}^{n} f_{i, k} u_{i, k}\right)\left(\sum_{j=1}^{n} f_{j} u_{j}\right)-\int_{\Omega \times[0, T]} e^{\rho}\left(\sum_{i=1}^{n} f_{i}(\Delta u)_{i}\right)\left(\sum_{j=1}^{n} f_{j} u_{j}\right) \\
\left(\text { since }\left(\sum_{j=1}^{n} f_{j} u_{j}\right)\left[\sum_{i=2}^{n}\left(\nabla f_{i}\right) u_{i}\right] \cdot \nu=0 \quad \text { on } \partial \Omega \times[0, T]\right) .
\end{gathered}
$$


Let us estimate

$$
\begin{aligned}
& -\int_{\Omega \times[0, T]} e^{\rho}\left(\sum_{k=1}^{n} \sum_{i=1}^{n} f_{i, k} u_{i k}\right)\left(\sum_{j=1}^{n} f_{j} u_{j}\right) \\
& =-\int_{\Omega \times[0, T]} e^{\rho}\left(\sum_{j=1}^{n} f_{j} u_{j}\right) \sum_{i=1}^{n} \nabla f_{i} \cdot \nabla u_{i} \\
& =\int_{\Omega \times[0, T]} e^{\rho}\left(\sum_{j=1}^{n} f_{j} u_{j}\right) \sum_{i=1}^{n}\left(\triangle f_{i}\right) u_{i}+\int_{\Omega \times[0, T]} \sum_{i=1}^{n} u_{i} \nabla\left[e^{\rho} \sum_{j=1}^{n} f_{j} u_{j}\right] \cdot \nabla f_{i} \\
& \text { ( since }\left(\sum_{j=1}^{n} f_{j} u_{j}\right) u_{i} \frac{\partial f_{i}}{\partial \nu}=0,1 \leq i \leq n \text {, on } \partial \Omega \times[0, T] \text { ) } \\
& =\int_{\Omega \times[0, T]} e^{\rho}\left(\sum_{i=1}^{n} u_{i} \Delta f_{i}\right)\left(\sum_{j=1}^{n} f_{j} u_{j}\right)+\int_{\Omega \times[0, T]} e^{\rho} \sum_{i=1}^{n} u_{i}\left(\sum_{k=1}^{n} \rho_{k} f_{i, k}\right)\left(\sum_{j=1}^{n} f_{j} u_{j}\right) \\
& +\int_{\Omega \times[0, T]} e^{\rho} \sum_{k=1}^{n}\left(\sum_{i=1}^{n} u_{i} f_{i, k}\right)\left(\sum_{j=1}^{n} f_{j} u_{j}\right)_{k} \\
& \leq \int_{\Omega \times[0, T]} e^{\rho}\left(\sum_{i=1}^{n} u_{i} \Delta f_{i}\right)\left(\sum_{j=1}^{n} f_{j} u_{j}\right)+\int_{\Omega \times[0, T]} e^{\rho} \sum_{i=1}^{n} u_{i}\left(\sum_{k=1}^{n} \rho_{k} f_{i, k}\right)\left(\sum_{j=1}^{n} f_{j} u_{j}\right) \\
& +\varepsilon \int_{\Omega \times[0, T]} e^{\rho}\left|\nabla \sum_{j=1}^{n} f_{j} u_{j}\right|^{2}+\frac{1}{4 \varepsilon} \int_{\Omega \times[0, t]} e^{\rho} \sum_{k=1}^{n}\left(\sum_{i=1}^{n} u_{i} f_{i, k}\right)^{2} .
\end{aligned}
$$

Put (1.22) into (1.21), we get

$$
\begin{aligned}
& \int_{\Omega \times[0, T]} e^{\rho}\left|\nabla \sum_{i=1}^{n} f_{i} u_{i}\right|^{2} \\
\leq & 2 \varepsilon \int_{\Omega \times[0, T]} e^{\rho}\left|\nabla \sum_{j=1}^{n} f_{j} u_{j}\right|^{2}+\frac{1}{4 \varepsilon} \int_{\Omega \times[0, T]} e^{\rho}|\nabla \rho|^{2}\left(\sum_{j=1}^{n} f_{j} u_{j}\right)^{2} \\
& +\frac{1}{4 \varepsilon} \int_{\Omega \times[0, T]} e^{\rho}\left(\sum_{k=1}^{n}\left(\sum_{i=1}^{n} f_{i, k} u_{i}\right)^{2}+2 \int_{\Omega \times[0, T]} e^{\rho}\left(\sum_{i, k=1}^{n} \rho_{k} f_{i, k} u_{i}\right)\left(\sum_{j=1}^{n} f_{j} u_{j}\right)\right. \\
+ & \int_{\Omega \times[0, T]} e^{\rho}\left(\sum_{i=1}^{n}\left(\triangle f_{i}\right) u_{i}\right)\left(\sum_{j=1}^{n} f_{j} u_{j}\right)+\int_{\Omega \times[0, T]} \sum_{k=1}^{n} e^{\rho}\left(\sum_{i=1}^{n} f_{i, k} u_{i}\right)\left(\sum_{j=1}^{n} f_{j} u_{j}\right)_{k} \\
- & \int_{\Omega \times[0, T]} e^{\rho}\left(\sum_{i=1}^{n} f_{i}(\triangle u)_{i}\right)\left(\sum_{j=1}^{n} f_{j} u_{j}\right) \\
\leq & 3 \varepsilon \int_{\Omega \times[0, T]} e^{\rho}\left|\nabla \sum_{j=1}^{n} f_{j} u_{j}\right|^{2}+\frac{1}{4 \varepsilon} \int_{\Omega \times[0, T]} e^{\rho}|\nabla \rho|^{2}\left(\sum_{j=1}^{n} f_{j} u_{j}\right)^{2} \\
+ & \frac{1}{2 \varepsilon} \int_{\Omega \times[0, T]} e^{\rho} \sum_{k=1}^{n}\left(\sum_{i=1}^{n} f_{i, k} u_{i}\right)^{2}+2 \int_{\Omega \times[0, T]} e^{\rho}\left(\sum_{i, k=1}^{n} \rho_{k} f_{i, k} u_{i}\right)\left(\sum_{j=1}^{n} f_{j} u_{j}\right) \\
+ & \int_{\Omega \times[0, T]} e^{\rho}\left(\sum_{i=1}^{n}\left(\triangle f_{i}\right) u_{i}\right)\left(\sum_{j=1}^{n} f_{j} u_{j}\right)-\int_{\Omega \times[0, T]} e^{\rho}\left(\sum_{i=1}^{n} f_{i}(\triangle u)_{i}\right)\left(\sum_{j=1}^{n} f_{j} u_{j}\right) .
\end{aligned}
$$


Hence,

$$
\begin{aligned}
& (1-3 \varepsilon) \int_{\Omega \times[0, T]} e^{\rho}\left|\nabla \sum_{j=1}^{n} f_{j} u_{j}\right|^{2} \\
\leq & \frac{1}{4 \varepsilon} \int_{\Omega \times[0, T]} e^{\rho}|\nabla \rho|^{2}\left(\sum_{j=1}^{n} f_{j} u_{j}\right)^{2}+\frac{1}{2 \varepsilon} \int_{\Omega \times[0, T]} e^{\rho} \sum_{k=1}^{n}\left(\sum_{i=1}^{n} f_{i, k} u_{i}\right)^{2} \\
+ & 2 \int_{\Omega \times[0, T]} e^{\rho}\left(\sum_{i, k=1}^{n} \rho_{k} f_{i, k} u_{i}\right)\left(\sum_{j=1}^{n} f_{j} u_{j}\right)+\int_{\Omega \times[0, T]} e^{\rho}\left(\sum_{i=1}^{n} u_{i} \Delta f_{i}\right)\left(\sum_{j=1}^{n} f_{j} u_{j}\right) \\
- & \int_{\Omega \times[0, T]} e^{\rho}\left(\sum_{i=1}^{n} f_{i}(\triangle u)_{i}\right)\left(\sum_{j=1}^{n} f_{j} u_{j}\right) .
\end{aligned}
$$

Put (1.20) and (1.24) into (1.15), we get

$$
\begin{aligned}
& \text { (1.24) } \int_{\Omega \times\{T\}} e^{\rho}(\Delta u)^{2}-\int_{\Omega \times\{0\}} e^{\rho}(\Delta u)^{2} \\
& \leq \int_{\Omega \times[0, T]} e^{\rho} \rho_{t}(\Delta u)^{2}-2 \int_{\Omega \times[0, T]} e^{\rho} \Delta u \nabla \rho \cdot \nabla(\Delta u)+\varepsilon \int_{\Omega \times[0, T]} e^{\rho}(\Delta u)^{2}|\nabla \rho|^{2}
\end{aligned}
$$$$
+\frac{2}{\varepsilon}(1-3 \varepsilon)^{-1} \frac{1}{4 \varepsilon} \int_{\Omega \times[0, T]} e^{\rho}|\nabla \rho|^{2}\left(\sum_{j=1}^{n} f_{j} u_{j}\right)^{2}
$$$$
+\frac{2}{\varepsilon}(1-3 \varepsilon)^{-1} \frac{1}{2 \varepsilon} \int_{\Omega \times[0, T]} e^{\rho} \sum_{k=1}^{n}\left(\sum_{i=1}^{n} f_{i, k} u_{i}\right)^{2}
$$$$
+\frac{2}{\varepsilon}(1-3 \varepsilon)^{-1} \cdot 2 \int_{\Omega \times[0, T]} e^{\rho}\left(\sum_{i, k=1}^{n} \rho_{k} f_{i, k} u_{i}\right)\left(\sum_{j=1}^{n} f_{j} u_{j}\right)
$$$$
+\frac{2}{\varepsilon}(1-3 \varepsilon)^{-1} \int_{\Omega \times[0, T]} e^{\rho}\left(\sum_{i=1}^{n} u_{i} \triangle f_{i}\right)\left(\sum_{j=1}^{n} f_{j} u_{j}\right)
$$$$
-\frac{2}{\varepsilon}(1-3 \varepsilon)^{-1} \int_{\Omega \times[0, T]} e^{\rho}\left(\sum_{i=1}^{n} f_{i}(\Delta u)_{i}\right)\left(\sum_{j=1}^{n} f_{j} u_{j}\right)
$$$$
+\frac{2}{\varepsilon} \int_{\Omega \times[0, T]} e^{\rho}|\nabla(V u)|^{2}-2 \int_{\Omega \times[0, T]} e^{\rho}\left|\nabla(\Delta u)^{2}\right|
$$$$
-2 \int_{\Omega \times[0, T]} \sum_{k=1}^{n} e^{\rho}(\Delta u)_{k}\left[\sum_{i=1}^{n} u_{i}\left(f_{i, k}+f_{k, i}\right)-u_{k} \sum_{i=1}^{n} f_{i} \rho_{i}+f_{k} \sum_{i=1}^{n} u_{i} \rho_{i}-u_{k} \sum_{i=1}^{n} f_{i, i}\right]
$$$$
+\int_{\Omega \times[0, T]} e^{\rho} \sum_{i=1}^{n} \rho_{i} f_{i}(\triangle u)^{2}+\int_{\Omega \times[0, T]} e^{\rho}\left(\sum_{i=1}^{n} f_{i, i}\right)(\Delta u)^{2}
$$$$
+2 \int_{\Omega \times[0, T]} e^{\rho} \nabla(\triangle u) \cdot \nabla(V u) .
$$

Observe the following estimates

$$
-2 \int_{\Omega \times[0, T]} e^{\rho} \triangle u \nabla \rho \cdot \nabla(\triangle u)
$$




$$
\begin{aligned}
\leq 2 \delta_{1} \int_{\Omega \times[0, T]} e^{\rho}|\nabla(\Delta u)|^{2} & +\frac{1}{2 \delta_{1}} \int_{\Omega \times[0, T]} e^{\rho}(\Delta u)^{2}|\nabla \rho|^{2} \\
\int_{\Omega \times[0, T]} e^{\rho}|\nabla \rho|^{2}\left(\sum_{j=1}^{n} f_{j} u_{j}\right)^{2} & \leq \int_{\Omega \times[0, T]} e^{\rho}|\nabla \rho|^{2}|f|^{2}|\nabla u|^{2} \\
\int_{\Omega \times[0, T]} e^{\rho} \sum_{k=1}^{n}\left(\sum_{i=1}^{n} f_{i, k} u_{i}\right)^{2} & \leq \int_{\Omega \times[0, T]} e^{\rho} \sum_{k=1}^{n} \sum_{i=1}^{n}\left(\frac{\partial f_{i}}{\partial x_{k}}\right)^{2}|\nabla u|^{2} \\
& =\int_{\Omega \times[0, T]} e^{\rho}|\nabla f|^{2}|\nabla u|^{2},
\end{aligned}
$$

where $|\nabla f|^{2}=\sum_{i=1}^{n}\left|\nabla f_{i}\right|^{2}$

$$
\begin{aligned}
& \int_{\Omega \times[0, T]} e^{\rho}\left(\sum_{i, k=1}^{n} \rho_{k} f_{i, k} u_{i}\right)\left(\sum_{j=1}^{n} f_{j} u_{j}\right)=\int_{\Omega \times[0, T]} e^{\rho} \sum_{k=1}^{n}\left(\sum_{j=1}^{n} f_{j} u_{j}\right) \rho_{k} \sum_{i=1}^{n} f_{i, k} u_{i} \\
& \leq \quad \frac{1}{2} \int_{\Omega \times[0, T]} e^{\rho}\left(\sum_{j=1}^{n} f_{j} u_{j}\right)^{2} \sum_{k=1}^{n} \rho_{k}^{2}+\frac{1}{2} \int_{\Omega \times[0, T]} e^{\rho} \sum_{k=1}^{n}\left(\sum_{i=1}^{n} f_{i, k} u_{i}\right)^{2}
\end{aligned}
$$$$
=O\left(\int_{\Omega \times[0, T]} e^{\rho}|f|^{2}|\nabla u|^{2}|\nabla \rho|^{2}+\int_{\Omega \times[0, T]} e^{\rho}|\nabla f|^{2}|\nabla u|^{2}\right)
$$

$$
\begin{gathered}
\int_{\Omega \times[0, T]} e^{\rho}\left(\sum_{i=1}^{n} u_{i} \Delta f_{i}\right)\left(\sum_{j=1}^{n} f_{j} u_{j}\right) \leq \int_{\Omega \times[0, T]} e^{\rho}|\nabla u|^{2}|f||\triangle f| \\
\text { where }|\triangle f|=\sqrt{\sum_{i=1}^{n}\left(\triangle f_{i}\right)^{2}}
\end{gathered}
$$

$$
\begin{gathered}
\int_{\Omega \times[0, T]} e^{\rho}|f|^{2}\left(\sum_{j=1}^{n} f_{j} u_{j}\right)^{2} \leq \int_{\Omega \times[0, T]} e^{\rho}|f|^{4}|\nabla u|^{2} \\
-\int_{\Omega \times[0, T]} e^{\rho}\left(\sum_{i=1}^{n} f_{i}(\Delta u)_{i}\right)\left(\sum_{j=1}^{n} f_{j} u_{j}\right)=-\int_{\Omega \times[0, T]} e^{\rho} \sum_{i=1}^{n}\left[\left(\sum_{j=1}^{n} f_{j} u_{j}\right) f_{i}\right](\Delta u)_{i} \\
\leq \delta_{2} \int_{\Omega \times[0, T]} e^{\rho} \sum_{i=1}^{n}\left[(\Delta u)_{i}\right]^{2}+\frac{1}{4 \delta_{2}} \int_{\Omega \times[0, T]} e^{\rho} \sum_{i=1}^{n}\left(\sum_{j=1}^{n} f_{j} u_{j}\right)^{2} f_{i}^{2} \\
\leq \delta_{2} \int_{\Omega \times[0, T]} e^{\rho}|\nabla(\Delta u)|^{2}+\frac{1}{4 \delta_{2}} \int_{\Omega \times[0, T]} e^{\rho}|f|^{4}|\nabla u|^{2}
\end{gathered}
$$

$$
-\int_{\Omega \times[0, T]} e^{\rho} \sum_{k=1}^{n}(\Delta u)_{k} \sum_{i=1}^{n} u_{i}\left(f_{i, k}+f_{k, i}\right)
$$

$$
\begin{array}{cc}
\leq & \delta_{3} \int_{\Omega \times[0, T]} e^{\rho} \sum_{k=1}^{n}\left[(\Delta u)_{k}\right]^{2}+\frac{1}{4 \delta_{3}} \int_{\Omega \times[0, T]} e^{\rho} \sum_{k=1}^{n}\left[\sum_{i=1}^{n} u_{i}\left(f_{i, k}+f_{k, i}\right)\right]^{2} \\
= & \delta_{3} \int_{\Omega \times[0, T]} e^{\rho}|\nabla(\Delta u)|^{2}+O\left(\int_{\Omega \times[0, T]} e^{\rho}|\nabla u|^{2}|\nabla f|^{2}\right) \\
& \quad-\int_{\Omega \times[0, T]} e^{\rho} \sum_{k=1}^{n}(\Delta u)_{k}\left(-u_{k} \sum_{i=1}^{n} f_{i} \rho_{i}+f_{k} \sum_{i=1}^{n} u_{i} \rho_{i}\right)
\end{array}
$$


1090

S. T. YAU AND S. S. T. YAU

$\leq \delta_{4} \int_{\Omega \times[0, T]} e^{\rho} \sum_{k=1}^{n}\left[(\Delta u)_{k}\right]^{2}+\frac{1}{4 \delta_{4}} \int_{\Omega \times[0, T]} e^{\rho} \sum_{k=1}^{n}\left(-u_{k} \sum_{i=1}^{n} f_{i} \rho_{i}+f_{k} \sum_{i=1}^{n} u_{i} \rho_{i}\right)^{2}$

$=\delta_{4} \int_{\Omega \times[0, T]} e^{\rho}|\nabla(\Delta u)|^{2}+O\left(\int_{\Omega \times[0, T]} e^{\rho}|\nabla u|^{2}|f|^{2}|\nabla \rho|^{2}\right)$

$(1.34)$

$-\int_{\Omega \times[0, T]} e^{\rho} \sum_{k=1}^{n}(\Delta u)_{k} u_{k} \sum_{i=1}^{n} f_{i, i}$
$\leq \quad \delta_{5} \int_{\Omega \times[0, T]} e^{\rho} \sum_{k=1}^{n}\left((\Delta u)_{k}\right)^{2}+\frac{1}{4 \delta_{5}} \int_{\Omega \times[0, T]} e^{\rho} \sum_{k=1}^{n}\left(u_{k} \sum_{i=1}^{n} f_{i, i}\right)^{2}$
$\leq \quad \delta_{5} \int_{\Omega \times[0, T]} e^{\rho}|\nabla(\Delta u)|^{2}+\frac{1}{4 \delta_{5}} \int_{\Omega \times[0, T]} e^{\rho}|\nabla u|^{2}\left(\sum_{i=1}^{n} f_{i, i}\right)^{2}$

$$
\begin{aligned}
& 2 \int_{\Omega \times[0, T]} e^{\rho} \nabla(\Delta u) \cdot \nabla(V u) \\
& \leq 2 \delta_{6} \int_{\Omega \times[0, T]} e^{\rho}|\nabla(\Delta u)|^{2}+\frac{1}{2 \delta_{6}} \int_{\Omega \times[0, T]} e^{\rho}|\nabla(V u)|^{2}
\end{aligned}
$$

Put the estimates $(1.26)-(1.36)$ into $(1.25)$, we get

$$
\begin{aligned}
& \int_{\Omega \times\{T\}} e^{\rho}(\Delta u)^{2}-\int_{\Omega \times\{0\}} e^{\rho}(\Delta u) \\
\leq & \int_{\Omega \times[0, T]} e^{\rho} \rho_{t}(\Delta u)^{2}+2 \delta_{1} \int_{\Omega \times[0, T]} e^{\rho}|\nabla(\Delta u)|^{2}+\frac{1}{2 \delta_{1}} \int_{\Omega \times[0, T]} e^{\rho}(\Delta u)|\nabla \rho|^{2} \\
+ & \int_{\Omega \times[0, T]} e^{\rho}(\Delta u)^{2}|\nabla \rho|^{2}+\frac{1}{2 \varepsilon^{2}(1-3 \varepsilon)} \int_{\Omega \times[0, T]} e^{\rho}|\nabla \rho|^{2}|f|^{2}|\nabla u|^{2} \\
+ & \frac{1}{\varepsilon^{2}(1-3 \varepsilon)} \int_{\Omega \times[0, T]} e^{\rho}|\nabla f|^{2}|\nabla u|^{2}+\frac{4}{\varepsilon(1-3 \varepsilon)} O\left(\int_{\Omega \times[0, T]} e^{\rho}|f|^{2}|\nabla u|^{2}|\nabla \rho|^{2}\right. \\
+ & \left.\int_{\Omega \times[0, T]} e^{\rho}|\nabla f|^{2}|\nabla u|^{2}\right)+\frac{2}{\varepsilon(1-3 \varepsilon)} \int_{\Omega \times[0, T]} e^{\rho}|\nabla u|^{2}|f||\Delta f| \\
+ & \frac{2}{\varepsilon(1-3 \varepsilon)} \delta_{2} \int_{\Omega \times[0, T]} e^{\rho}|\nabla(\Delta u)|^{2}+\frac{2}{\varepsilon(1-3 \varepsilon)} \frac{1}{4 \delta_{2}} \int_{\Omega \times[0, T]} e^{\rho}|f|^{4}|\nabla u|^{2} \\
+ & \frac{2}{\varepsilon} \int_{\Omega \times[0, T]} e^{\rho}|\nabla(V u)|^{2}-2 \int_{\Omega \times[0, T]} e^{\rho}|\nabla(\Delta u)|^{2} \\
+ & 2 \delta_{3} \int_{\Omega \times[0, T]} e^{\rho}|\nabla(\Delta u)|^{2}+O\left(\int_{\Omega \times[0, T]} e^{\rho}|\nabla u|^{2}|\nabla f|^{2}\right) \\
+ & 2 \delta_{4} \int_{\Omega \times[0, T]} e^{\rho}|\nabla(\Delta u)|^{2}+O\left(\int_{\Omega \times[0, T]} e^{\rho}|\nabla u|^{2}|f|^{2}|\nabla \rho|^{2}\right) \\
+ & +2 \delta_{5} \int_{\Omega \times[0, T]} e^{\rho}|\nabla(\Delta u)|^{2}+\frac{1}{2 \delta_{5}} \int_{\Omega \times[0, T]} e^{\rho}|\nabla u|^{2}\left(\sum_{i=1}^{n} f_{i, i}\right)^{2} \\
+ & \int^{\rho} \sum^{n} \rho_{i} f_{i}(\Delta u)^{2}+\int_{\Omega \times[0, T]} e^{\rho}\left(\sum_{i=1}^{n} f_{i, i}\right)(\Delta u)^{2} \\
+ &
\end{aligned}
$$




$$
\begin{aligned}
& +2 \delta_{6} \int_{\Omega \times[0, T]} e^{\rho}|\nabla(\Delta u)|^{2}+\frac{1}{2 \delta_{6}} \int_{\Omega \times[0, T]} e^{\rho}|\nabla(V u)|^{2} \\
= & \int_{\Omega \times[0, T]} e^{\rho}(\Delta u)^{2}\left[\rho_{t}+\left(\varepsilon+\frac{1}{2 \delta_{1}}\right)|\nabla \rho|^{2}+\sum_{i=1}^{n} \rho_{i} f_{i}+\sum_{i=1}^{n} f_{i, i}\right] \\
& +\left(2 \delta_{1}+\frac{2 \delta_{2}}{\varepsilon(1-3 \varepsilon)}-2+2 \delta_{3}+2 \delta_{4}+2 \delta_{5}+2 \delta_{6}\right) \int_{\Omega \times[0, T]} e^{\rho}|\nabla(\Delta u)|^{2} \\
& +O\left(\int_{\Omega \times[0, T]} e^{\rho}|\nabla \rho|^{2}|f|^{2}|\nabla u|^{2}+\int_{\Omega \times[0, T]} e^{\rho}|\nabla f|^{2}|\nabla u|^{2}\right. \\
& +\int_{\Omega \times[0, T]} e^{\rho}|f||\nabla u||\Delta f|+\int_{\Omega \times[0, T]} e^{\rho}|f|^{4}|\nabla u|^{2} \\
& \left.+\int_{\Omega \times[0, T]} e^{\rho}|\nabla(V u)|^{2}+\int_{\Omega \times[0, T]} e^{\rho}|\nabla u|^{2}\left(\sum_{i=1}^{n} f_{i, i}\right)^{2}\right) .
\end{aligned}
$$

Let $\delta_{1}=\delta_{2}=\delta_{3}=\delta_{4}=\delta_{5}=\delta_{6}=\varepsilon^{2}$ with $2-10 \varepsilon^{2}-\frac{2 \varepsilon}{1-3 \varepsilon}>0$. Since $\frac{\partial \rho}{\partial t}+(\varepsilon+$ $\left.\frac{1}{2 \varepsilon^{2}}\right)|\nabla \rho|^{2}+\sum_{i=1}^{n} \rho_{i} f_{i}+\sum_{i=1}^{n} f_{i, i} \leq 0$, for $0 \leq t \leq T$, by assumption, (1.36) implies (1.34).

In view of Theorem 1.1, we are interested in constructing functions $g, h$ and $\rho$ which satisfy the conditions (i), (ii) and (iii) in Theorem 1.1. The following theorem is very useful in this regard.

TheOREM 1.2. Let $k: \Omega \times \mathbb{R} \rightarrow \mathbb{R}^{n}$ and $F: \Omega \times \mathbb{R} \rightarrow \mathbb{R}$ be a possibly time dependent vector field and function respectively. Fix a point $\left(x_{0}, t_{0}\right) \in \Omega \times \mathbb{R}$. Let $(x, t)$ be an arbitrary point in $\Omega \times \mathbb{R}$ with $t>t_{0}$. Let $\mathcal{P}=\{$ differentiable path $\sigma=$ $\left(\sigma_{1}, \sigma_{2}\right):[0,1] \rightarrow \Omega \times \mathbb{R}$ such that $\sigma(0)=\left(\sigma_{1}(0), \sigma_{2}(0)\right)=\left(x_{0}, t_{0}\right), \sigma(1)=(x, t), \sigma_{2}(s)$ is linear in $s$ with $\sigma_{2}{ }^{\prime}(s) \geq 0$ and $\left.\int_{0}^{1} \sigma_{1}(s) d s=\frac{x_{0}+x}{2}\right\}$. Define

$$
\begin{gathered}
E_{\varepsilon}(\sigma)=\frac{1}{4} \int_{0}^{1} \frac{\left|\dot{\sigma}_{1}\right|^{2}}{\frac{\partial \sigma_{2}}{\partial s}+\varepsilon}-\frac{1}{2} \int_{0}^{1} \sum_{i=1}^{n} k_{i}\left(\sigma_{1}(s), \sigma_{2}(s)\right) \frac{d \sigma_{1}^{i}}{d s}+\int_{0}^{1} F\left(\sigma_{1}(s), \sigma_{2}(s)\right) \frac{d \sigma_{2}}{d s} \\
d_{\varepsilon}(x, t)=\min _{\sigma \in \mathcal{P}} E_{\varepsilon}(\sigma)
\end{gathered}
$$

Let $\sigma$ be the curve that minimizes the functional $E_{\varepsilon}(\sigma)$. Then the following equations hold for almost all $(x, t)$.

$$
\begin{aligned}
\nabla d_{\varepsilon}(x, t) & =\frac{1}{2} \frac{\dot{\sigma}_{1}(1)}{\frac{\partial \sigma_{2}}{\partial s}(1)+\varepsilon}-\frac{1}{2} k(x, t) \\
\sum_{i=1}^{n} k_{i}(x, t)\left(d_{\varepsilon}\right)_{i}(x, t) & =-\frac{1}{2}|k(x, t)|^{2}+\frac{1}{2}\left(\sum_{i=1}^{n} k_{i}(x, t) \frac{d \sigma_{1}^{i}}{d s}(1)\right)\left(\frac{d \sigma_{2}}{d s}(1)+\varepsilon\right)^{-1} \\
\frac{\partial d_{\varepsilon}}{\partial t}(x, t) & =-\frac{1}{4} \frac{\left|\dot{\sigma}_{1}(1)\right|^{2}}{\left(\frac{\partial \sigma_{2}}{\partial s}(1)+\varepsilon\right)^{2}}+F(x, t)
\end{aligned}
$$

In particular $d_{\varepsilon}(x, t)$ satisfies the following equation

1.40) $\frac{\partial d_{\varepsilon}}{\partial t}(x, t)+\left|\nabla d_{\varepsilon}(x, t)\right|^{2}+\sum_{i=1}^{n} k_{i}(x, t)\left(d_{\varepsilon}\right)_{i}(x, t)=F(x, t)-\frac{|k(x, t)|^{2}}{4}$. 
Proof. If $\sigma=\left(\sigma_{1}, \sigma_{2}\right) \in \mathcal{P}$ is the path that realizes the minimum of $E_{\varepsilon}(\sigma)$, then it has to satisfy the Euler-Lagrange equations which we shall derive explicity as follows. Consider an one parameter family of path in $\mathcal{P}$ :

$$
\begin{aligned}
& \alpha:(-\varepsilon, \varepsilon) \times[0,1] \longrightarrow \Omega \times \mathbb{R} \\
& \alpha(0, s)=\sigma(s), \quad \forall s \in[0,1] \\
& \alpha(v, 0)=\left(x_{0}, t_{0}\right), \quad \forall v \in(-\varepsilon, \varepsilon) \\
& \alpha(v, 1)=(x, t), \quad \forall v \in(-\varepsilon, \varepsilon)
\end{aligned}
$$

Denote $\bar{\alpha}(v)(s)=\left(\bar{\alpha}_{1}(v)(s), \bar{\alpha}_{2}(v)(s)\right):=\alpha(v, s)=\left(\alpha_{1}(v, s), \alpha_{2}(v, s)\right)$ and $\bar{\alpha}_{1}(v)^{i}:=i$ th component of $\bar{\alpha}_{1}(v)$.

$$
\begin{aligned}
& \left.\frac{d}{d v} E_{\varepsilon}(\bar{\alpha}(v))\right|_{v=0}=\frac{d}{d v}\left\{\frac{1}{4} \int_{0}^{1} \frac{\left|\frac{\dot{\alpha}_{1}}{\alpha_{1}}(v)(s)\right|^{2}}{\frac{\partial \bar{\alpha}_{2}(v)(s)}{\partial s}+\varepsilon}-\frac{1}{2} \int_{0}^{1} \sum_{i=1}^{n} k_{i}\left(\bar{\alpha}(v)(s), \bar{\alpha}_{2}(v)(s)\right) \frac{d \bar{\alpha}_{1}(v)^{i}}{d s}(s)\right. \\
& \left.+\int_{0}^{1} F\left(\bar{\alpha}_{1}(v)(s), \bar{\alpha}_{2}(v)(s)\right) \frac{d \bar{\alpha}_{2}(v)}{d s}(s)\right\}\left.\right|_{v=0} \\
& =\frac{1}{4} \int_{0}^{1}\left[\left.\frac{2 \frac{\partial \alpha_{1}}{\partial s}(v, s) \frac{\partial^{2} \alpha_{1}}{\partial v \partial s}(v, s)}{\frac{\partial \alpha_{2}}{\partial s}(v, s)+\varepsilon}\right|_{v=0}-\left.\frac{\left|\frac{\partial \alpha_{1}}{\partial s}(v, s)\right|^{2} \frac{\partial^{2} \alpha_{2}}{\partial v \partial s}(v, s)}{\left(\frac{\partial \alpha_{2}}{\partial s}(v, s)+\varepsilon\right)^{2}}\right|_{v=0}\right] \\
& -\frac{1}{2} \int_{0}^{1} \sum_{i=1}^{n}\left[\sum_{j=1}^{n} \frac{\partial k_{i}}{\partial x_{j}}\left(\bar{\alpha}_{1}(v)(s), \bar{\alpha}_{2}(v)(s)\right) \frac{\partial \alpha_{1}^{j}}{\partial v}(v, s)\right. \\
& \left.+\frac{\partial k_{i}}{\partial t}\left(\bar{\alpha}_{1}(v)(s), \bar{\alpha}_{2}(v)(s)\right) \frac{\partial \alpha_{2}}{\partial v}(v, s)\right]\left.\frac{d \bar{\alpha}_{1}(v)^{i}}{d s}(s)\right|_{v=0} \\
& -\left.\frac{1}{2} \int_{0}^{1} \sum_{i=1}^{n} k_{i}\left(\bar{\alpha}_{1}(v)(s), \bar{\alpha}_{2}(v)(s)\right) \frac{\partial^{2} \alpha_{1}^{i}}{\partial v \partial s}(v, s)\right|_{v=0} \\
& +\int_{0}^{1}\left[\sum_{j=1}^{n} \frac{\partial F}{\partial x_{j}}\left(\bar{\alpha}_{1}(v)(s), \bar{\alpha}_{2}(v)(s)\right) \frac{\partial \alpha_{1}^{j}}{\partial v}(v, s)\right. \\
& \left.+\frac{\partial F}{\partial t}\left(\bar{\alpha}_{1}(v)(s), \bar{\alpha}_{2}(v)(s)\right) \frac{\partial \alpha_{2}}{\partial v}(v, s)\right]\left.\frac{\partial \alpha_{2}}{\partial s}(v, s)\right|_{v=0} \\
& +\left.\int_{0}^{1} F\left(\bar{\alpha}_{1}(v)(s), \bar{\alpha}_{2}(v)(s)\right) \frac{\partial^{2} \alpha_{2}}{\partial v \partial s}(v, s)\right|_{v=0} \\
& =\frac{1}{4} \int_{0}^{1}\left[\frac{2 \frac{\partial \alpha_{1}}{\partial s}(0, s) \frac{\partial^{2} \alpha_{1}}{\partial v \partial s}(0, s)}{\frac{\partial \alpha_{2}}{\partial s}(0, s)+\varepsilon}-\frac{\left|\frac{\partial \alpha_{1}}{\partial s}(0, s)\right|^{2} \frac{\partial^{2} \alpha_{2}}{\partial v \partial s}(0, s)}{\left(\frac{\partial \alpha_{2}}{\partial s}(0, s)+\varepsilon\right)^{2}}\right] \\
& -\frac{1}{2} \int_{0}^{1} \sum_{i=1}^{n}\left[\sum_{j=1}^{n} \frac{\partial k_{i}}{\partial x_{j}}\left(\alpha_{1}(0, s), \alpha_{2}(0, s)\right) \frac{\partial \alpha_{1}^{j}}{\partial v}(0, s)\right. \\
& \left.+\frac{\partial k_{i}}{\partial t}\left(\alpha_{1}(0, s), \alpha_{2}(0, s)\right) \frac{\partial \alpha_{2}}{\partial v}(0, s)\right] \frac{d \alpha_{1}^{i}(0, s)}{d s} \\
& -\frac{1}{2} \int_{0}^{1} \sum_{i=1}^{n} k_{i}\left(\alpha_{1}(0, s), \alpha_{2}(0, s)\right) \frac{\partial^{2} \alpha_{1}^{i}}{\partial v \partial s}(0, s) \\
& +\int_{0}^{1}\left[\sum_{j=1}^{n} \frac{\partial F}{\partial x_{j}}\left(\alpha_{1}(0, s), \alpha_{2}(0, s)\right) \frac{\partial \alpha_{1}^{j}}{\partial v}(0, s)\right.
\end{aligned}
$$




$$
\begin{aligned}
& \left.+\frac{\partial F}{\partial t}\left(\alpha_{1}(0, s), \alpha_{2}(0, s)\right) \frac{\partial \alpha_{2}}{\partial v}(0, s)\right] \frac{\partial \alpha_{2}}{\partial s}(0, s) \\
& +\int_{0}^{1} F\left(\alpha_{1}(0, s), \alpha_{2}(0, s)\right) \frac{\partial^{2} \alpha_{2}}{\partial v \partial s}(0, s) \\
& =\frac{1}{2} \int_{0}^{1} \frac{\frac{\partial \alpha_{1}}{\partial s}(0, s)}{\frac{\partial \alpha_{2}}{\partial s}(0, s)+\varepsilon} \frac{d}{d s}\left(\frac{\partial \alpha_{1}}{\partial v}(0, s)\right) \\
& -\frac{1}{4} \int_{0}^{1} \frac{\left|\frac{\partial \alpha_{1}}{\partial s}(0, s)\right|^{2}}{\left(\frac{\partial \alpha_{2}}{\partial s}(0, s)+\varepsilon\right)^{2}} \frac{d}{d s}\left(\frac{\partial \alpha_{2}}{\partial v}(0, s)\right) \\
& \left.-\frac{1}{2} \int_{0}^{1} \sum_{i=1}^{n} \sum_{j=1}^{n} \frac{\partial k_{i}}{\partial x_{j}}\left(\alpha_{1}(0, s)\right), \alpha_{2}(0, s)\right) \frac{\partial \alpha_{1}^{j}(0, s)}{\partial v} \frac{\partial \alpha_{1}^{i}(0, s)}{\partial s} \\
& \left.-\frac{1}{2} \int_{0}^{1} \sum_{i=1}^{n} \frac{\partial k_{i}}{\partial t}\left(\alpha_{1}(0, s)\right), \alpha_{2}(0, s)\right) \frac{\partial \alpha_{2}}{\partial v}(0, s) \frac{\partial \alpha_{1}^{i}(0, s)}{\partial s} \\
& -\frac{1}{2} \int_{0}^{1} \sum_{i=1}^{n} k_{i}\left(\alpha_{1}(0, s), \alpha_{2}(0, s)\right) \frac{d}{d s}\left(\frac{\partial \alpha_{1}^{i}(0, s)}{\partial v}\right) \\
& +\int_{0}^{1} \sum_{j=1}^{n} \frac{\partial F}{\partial x_{j}}\left(\alpha_{1}(0, s), \alpha_{2}(0, s)\right) \frac{\partial \alpha_{1}^{j}(0, s)}{\partial v} \frac{\partial \alpha_{2}(0, s)}{\partial s} \\
& +\int_{0}^{1} \frac{\partial F}{\partial t}\left(\alpha_{1}(0, s), \alpha_{2}(0, s)\right) \frac{\partial \alpha_{2}}{\partial v}(0, s) \frac{\partial \alpha_{2}}{\partial s}(0, s) \\
& +\int_{0}^{1} F\left(\alpha_{1}(0, s), \alpha_{2}(0, s)\right) \frac{d}{d s}\left(\frac{\partial \alpha_{2}}{\partial v}(0, s)\right) \\
& =-\frac{1}{2} \int_{0}^{1} \frac{\partial}{\partial s}\left[\frac{\frac{\partial \alpha_{1}}{\partial s}(0, s)}{\frac{\partial \alpha_{2}}{\partial s}(0, s)+\varepsilon}\right] \cdot \frac{\partial \alpha_{1}}{\partial v}(0, s)+\left.\frac{1}{2} \frac{\frac{\partial \alpha_{1}}{\partial s}(0, s)}{\frac{\partial \alpha_{2}}{\partial s}(0, s)+\varepsilon} \frac{\partial \alpha_{1}}{\partial v}(0, s)\right|_{s=0} ^{s=1} \\
& +\frac{1}{4} \int_{0}^{1} \frac{\partial}{\partial s}\left[\frac{\left|\frac{\partial \alpha_{1}}{\partial s}(0, s)\right|^{2}}{\left(\frac{\partial \alpha_{2}}{\partial s}(0, s)+\varepsilon\right)^{2}}\right] \cdot \frac{\partial \alpha_{2}}{\partial v}(0, s) \\
& -\left.\frac{1}{4} \frac{\left|\frac{\partial \alpha_{1}}{\partial s}(0, s)\right|^{2}}{\left(\frac{\partial \alpha_{2}}{\partial s}(0, s)+\varepsilon\right)^{2}} \frac{\partial \alpha_{2}}{\partial s}(0, s)\right|_{s=0} ^{s=1} \\
& -\frac{1}{2} \int_{0}^{1} \sum_{i=1}^{n} \sum_{j=1}^{n} \frac{\partial k_{i}}{\partial x_{\jmath}}\left(\alpha_{1}(0, s), \alpha_{2}(0, s)\right) \frac{\partial \alpha_{1}^{i}}{\partial s}(0, s) \frac{\partial \alpha_{1}^{j}}{\partial v}(0, s) \\
& -\frac{1}{2} \int_{0}^{1} \sum_{i=1}^{n} \frac{\partial k_{i}}{\partial t}\left(\alpha_{1}(0, s), \alpha_{2}(0, s)\right) \frac{\partial \alpha_{1}^{i}}{\partial s}(0, s) \frac{\partial \alpha_{2}}{\partial v}(0, s) \\
& +\frac{1}{2} \int_{0}^{1} \sum_{i=1}^{n} \sum_{j=1}^{n} \frac{\partial k_{i}}{\partial x_{j}}\left(\alpha_{1}(0, s), \alpha_{2}(0, s)\right) \frac{\partial \alpha_{1}^{j}(0, s)}{\partial s} \frac{\partial \alpha_{1}^{i}}{\partial v}(0, s) \\
& +\frac{1}{2} \int_{0}^{1} \sum_{i=1}^{n} \frac{\partial k_{i}}{\partial t}\left(\alpha_{1}(0, s), \alpha_{2}(0, s)\right) \frac{\partial \alpha_{2}}{\partial s}(0, s) \frac{\partial \alpha_{1}^{i}}{\partial v}(0, s) \\
& -\left.\frac{1}{2} \sum_{i=1}^{n} k_{i}\left(\alpha_{1}(0, s), \alpha_{2}(0, s)\right) \frac{\partial \alpha_{1}^{i}(0, s)}{\partial v}\right|_{s=0} ^{s=1}
\end{aligned}
$$




$$
\begin{aligned}
& +\int_{0}^{1} \sum_{j=1}^{n} \frac{\partial F}{\partial x_{j}}\left(\alpha_{1}(0, s), \alpha_{2}(0, s)\right) \frac{\partial \alpha_{2}(0, s)}{\partial s} \frac{\partial \alpha_{1}^{j}(0, s)}{\partial v} \\
& +\int_{0}^{1} \frac{\partial F}{\partial t}\left(\alpha_{1}(0, s), \alpha_{2}(0, s)\right) \frac{\partial \alpha_{2}}{\partial s}(0, s) \frac{\partial \alpha_{2}}{\partial s}(0, s) \\
& -\int_{0}^{1} \sum_{j=1}^{n} \frac{\partial F}{\partial x_{i}}\left(\alpha_{1}(0, s), \alpha_{2}(0, s)\right) \frac{\partial \alpha_{1}^{i}(0, s)}{\partial s} \frac{\partial \alpha_{2}}{\partial v}(0, s) \\
& -\int_{0}^{1} \frac{\partial F}{\partial t}\left(\alpha_{1}(0, s), \alpha_{2}(0, s)\right) \frac{\partial \alpha_{2}}{\partial s}(0, s) \frac{\partial \alpha_{2}}{\partial v}(0, s) \\
& +\left.F\left(\alpha_{1}(0, s), \alpha_{2}(0, s)\right) \frac{\partial \alpha_{2}}{\partial v}(0, s)\right|_{s=0} ^{s=1} \\
& =\frac{1}{2} \int_{0} \frac{\partial}{\partial s}\left[\frac{\frac{\partial \alpha_{1}}{\partial s}(0, s)}{\frac{\partial \alpha_{2}}{\partial s}(0, s)+\varepsilon}\right] \frac{\partial \alpha_{1}}{\partial v}(0, s) \\
& -\frac{1}{2} \int_{0}^{1} \sum_{i=1}^{n} \sum_{j=1}^{n} \frac{\partial k_{i}}{\partial x_{j}}\left(\alpha_{1}(0, s), \alpha_{2}(0, s)\right) \frac{\partial \alpha_{1}^{i}}{\partial s}(0, s) \frac{\partial \alpha_{1}^{j}}{\partial v}(0, s) \\
& +\frac{1}{2} \int_{0}^{1} \sum_{i=1}^{n} \sum_{j=1}^{n} \frac{\partial k_{i}}{\partial x_{j}}\left(\alpha_{1}(0, s), \alpha_{2}(0, s)\right) \frac{\partial \alpha_{1}^{j}}{\partial s}(0, s) \frac{\partial \alpha_{1}^{i}}{\partial v}(0, s) \\
& +\frac{1}{2} \int_{0}^{1} \sum_{i=1}^{n} \frac{\partial k_{i}}{\partial t}\left(\alpha_{1}(0, s), \alpha_{2}(0, s)\right) \frac{\partial \alpha_{2}}{\partial s}(0, s) \frac{\partial \alpha_{1}^{i}}{\partial v}(0, s) \\
& \left.+\frac{1}{2} \int_{0}^{1} \sum_{j=1}^{n} \frac{\partial F}{\partial x_{j}}\left(\alpha_{1}(0, s), \alpha_{2}(0, s)\right) \frac{\partial \alpha_{2}}{\partial s}(0, s)\right) \frac{\partial \alpha_{1}^{j}}{\partial v}(0, s) \\
& +\frac{1}{4} \int_{0}^{1} \frac{\partial}{\partial s}\left[\frac{\left|\frac{\partial \alpha_{1}}{\partial s}(0, s)\right|^{2}}{\left(\frac{\partial \alpha_{2}}{\partial s}(0, s)+\varepsilon\right)^{2}}\right] \frac{\partial \alpha_{2}}{\partial v}(0, s) \\
& -\frac{1}{2} \int_{0}^{1} \sum_{i=1}^{n} \frac{\partial F}{\partial x_{i}}\left(\alpha_{1}(0, s), \alpha_{2}(0, s)\right) \frac{\partial \alpha_{1}^{i}}{\partial s}(0, s) \frac{\partial \alpha_{2}}{\partial v}(0, s) \\
& -\frac{1}{2} \int_{0}^{1} \sum_{i=1}^{n} \frac{\partial k_{i}}{\partial t}\left(\alpha_{1}(0, s), \alpha_{2}(0, s)\right) \frac{\partial \alpha_{1}^{i}}{\partial s}(0, s) \frac{\partial \alpha_{2}}{\partial v}(0, s)
\end{aligned}
$$

In order that $\sigma$ minimizes $E_{\varepsilon}(\sigma), \sigma$ must make the above integrals vanish for every $\eta(s)=\left(\frac{\partial \alpha_{1}}{\partial v}(0, s), \frac{\partial \alpha_{2}}{\partial v}(0, s)\right)$ which vanishes at $s=0$ and $s=1$. So we have derived the following Euler-Lagrange equations

$$
\begin{aligned}
& -\frac{1}{2} \frac{d}{d s}\left[\frac{\frac{d \sigma_{1}^{i}}{d s}(s)}{\frac{d \sigma_{2}}{d s}(s)+\varepsilon}\right]+\frac{1}{2}\left(\sum_{j=1}^{n} \frac{\partial k_{i}}{\partial x_{j}}\left(\sigma_{1}(s), \sigma_{2}(s)\right) \frac{\partial \sigma_{1}^{j}}{d s}(s)+\frac{\partial k_{i}}{\partial t}\left(\sigma_{1}(s), \sigma_{2}(s)\right) \frac{\partial \sigma_{2}}{\partial s}(s)\right) \\
& \begin{aligned}
&(1.41) \quad-\frac{1}{2} \sum_{j=1}^{n} \frac{\partial k_{j}}{\partial x_{i}}\left(\sigma_{1}(s), \sigma_{2}(s)\right) \frac{\partial \sigma_{1}^{j}}{d s}(s)+\frac{\partial F}{\partial x_{i}}\left(\sigma_{1}(s), \sigma_{2}(s)\right) \frac{\partial \sigma_{2}}{\partial s}(s)=0 \\
& \frac{1}{4} \frac{d}{d s}\left[\frac{\left|\frac{d \sigma_{1}}{d s}(s)\right|^{2}}{\left(\frac{d \sigma_{2}}{d s}(s)+\varepsilon\right)^{2}}\right]-\frac{1}{2} \sum_{i=1}^{n} \frac{\partial k_{i}}{\partial t}\left(\sigma_{1}(s), \sigma_{2}(s)\right) \frac{d \sigma_{1}^{i}}{d s}(s)
\end{aligned}
\end{aligned}
$$




$$
-\sum_{i=1}^{n} \frac{\partial F}{\partial x_{i}}\left(\sigma_{1}(s), \sigma_{2}(s) \frac{d \sigma_{1}^{i}}{d s}(s)=0\right.
$$

Now suppose $\sigma$ is the path in $\mathcal{P}$ which minimizes $E_{\varepsilon}(\sigma)$. Notices that $\sigma$ depends on $(x, t)$. Then

$$
\begin{aligned}
& \frac{\delta d_{\varepsilon}}{\delta x}(x, t)=\frac{\delta}{\delta x} E_{\varepsilon}(\sigma) \\
& =\frac{1}{4} \int_{0}^{1} \frac{\delta}{\delta x}\left[\frac{\left|\frac{d \sigma_{1}}{d s}(s)\right|^{2}}{\frac{d \sigma_{2}}{d s}(s)+\varepsilon}\right]-\frac{1}{2} \int_{0}^{1} \frac{\delta}{\delta x}\left[\sum_{i=1}^{n} k_{i}\left(\sigma_{1}(s), \sigma_{2}(s)\right) \frac{\partial \sigma_{1}^{i}}{\partial s}(s)\right] \\
& +\int_{0}^{1} \frac{\delta}{\delta x}\left[F\left(\sigma_{1}(s), \sigma_{2}(s)\right) \frac{\partial \sigma_{2}}{\partial s}\right] \\
& =\frac{1}{2} \int_{0}^{1} \frac{\left\langle\frac{\partial \sigma_{1}}{\partial s}, \frac{\delta \partial \sigma_{1}}{\delta x \partial s}>\right.}{\frac{\partial \sigma_{2}}{\partial s}+\varepsilon}-\frac{1}{4} \int_{0}^{1} \frac{\left|\frac{\partial \sigma_{1}}{\partial s}\right|^{2}}{\left(\frac{\partial \sigma_{2}}{\partial s}+\varepsilon\right)^{2}} \frac{\delta \partial \sigma_{2}}{\delta x \partial s} \\
& -\frac{1}{2} \int_{0}^{1} \sum_{i=1}^{n} \sum_{j=1}^{n} \frac{\partial k_{i}}{\partial x_{j}}\left(\sigma_{1}, \sigma_{2}\right) \frac{\delta \sigma_{1}^{j}}{\partial x} \frac{\partial \sigma_{1}^{i}}{\partial s}-\frac{1}{2} \int_{0}^{1} \sum_{i=1}^{n} \frac{\partial k_{i}}{\partial t}\left(\sigma_{1}, \sigma_{2}\right) \frac{\delta \sigma_{2}}{\delta x} \frac{\partial \sigma_{1}^{i}}{\partial s} \\
& -\frac{1}{2} \int_{0}^{1} \sum_{i=1}^{n} k_{i}\left(\sigma_{1}, \sigma_{2}\right) \frac{\delta \partial \sigma_{1}^{i}}{\delta x \partial s}+\int_{0}^{1} \sum_{i=1}^{n} \frac{\partial F}{\partial x_{i}}\left(\sigma_{1}, \sigma_{2}\right) \frac{\delta \sigma_{1}^{i}}{\delta x} \frac{\partial \sigma_{2}}{\partial s} \\
& +\int_{0}^{1} \frac{\partial F}{\partial t}\left(\sigma_{1}, \sigma_{2}\right) \frac{\delta \sigma_{2}}{\delta x} \frac{\partial \sigma_{2}}{\partial s}+\int_{0}^{1} F\left(\sigma_{1}, \sigma_{2}\right) \frac{\delta \partial \sigma_{2}}{\delta x \partial s} \\
& =\frac{1}{2} \int_{0}^{1} \sum_{i=1}^{n} \frac{\frac{\partial \sigma_{1}^{i}}{\partial s}}{\frac{\partial \sigma_{2}}{\partial s}+\varepsilon} \frac{d}{d s}\left(\frac{\delta \sigma_{1}^{i}}{\delta x}\right)-\frac{1}{4} \int_{0}^{1} \frac{\left|\frac{\partial \sigma_{1}}{\partial s}\right|^{2}}{\left(\frac{\partial \sigma_{2}}{\partial s}+\varepsilon\right)^{2}} \frac{d}{d s}\left(\frac{\delta \sigma_{2}}{\delta x}\right) \\
& -\frac{1}{2} \int_{0}^{1} \sum_{i=1}^{n} \sum_{j=1}^{n} \frac{\partial k_{i}}{\partial x_{j}}\left(\sigma_{1}, \sigma_{2}\right) \frac{\partial \sigma_{1}^{i}}{\partial s} \frac{\delta \sigma_{1}^{j}}{\delta x}-\frac{1}{2} \int_{0}^{1} \sum_{i=1}^{n} \frac{\partial k_{i}}{\partial t}\left(\sigma_{1}, \sigma_{2}\right) \frac{\partial \sigma_{1}^{i}}{\partial s} \frac{\delta \sigma_{2}}{\delta x} \\
& -\frac{1}{2} \int_{0}^{1} \sum_{i=1}^{n} k_{i}\left(\sigma_{1}, \sigma_{2}\right) \frac{d}{d s}\left(\frac{\partial \sigma_{1}^{i}}{\delta x}\right)+\int_{0}^{1} \sum_{i=1}^{n} \frac{\partial F}{\partial x_{i}}\left(\sigma_{1}, \sigma_{2}\right) \frac{\partial \sigma_{2}}{\partial s} \frac{\delta \sigma_{1}^{i}}{\delta x} \\
& +\int_{0}^{1} \frac{\partial F}{\partial t}\left(\sigma_{1}, \sigma_{2}\right) \frac{\partial \sigma_{2}}{\partial s} \frac{\delta \sigma_{2}}{\delta x}+\int_{0}^{1} F\left(\sigma_{1}, \sigma_{2}\right) \frac{d}{d s}\left(\frac{\delta \sigma_{2}}{\delta x}\right) \\
& =-\frac{1}{2} \int_{0}^{1} \sum_{i=1}^{n} \frac{\partial}{\partial s}\left(\frac{\frac{\partial \sigma_{1}^{i}}{\partial s}}{\frac{\partial \sigma_{2}}{\partial s}+\varepsilon}\right) \frac{\delta \sigma_{1}^{i}}{\delta x}+\left.\frac{1}{2} \sum_{i=1}^{n} \frac{\frac{\partial \sigma_{1}^{i}}{\partial s}}{\frac{\partial \sigma_{2}}{\partial s}+\varepsilon} \frac{\delta \sigma_{1}^{i}}{\delta x}\right|_{s=0} ^{s=1} \\
& +\frac{1}{4} \int_{0}^{1} \frac{\partial}{\partial s}\left[\frac{\left|\frac{\partial \sigma_{1}}{\partial s}\right|^{2}}{\left(\frac{\partial \sigma_{2}}{\partial s}+\varepsilon\right)^{2}}\right] \frac{\delta \sigma_{2}}{\delta x}-\left.\frac{1}{4} \frac{\left|\frac{\partial \sigma_{1}}{\partial s}\right|^{2}}{\left(\frac{\partial \sigma_{2}}{\partial s}+\varepsilon\right)^{2}} \frac{\delta \sigma_{2}}{\delta x}\right|_{s=0} ^{s=1} \\
& -\frac{1}{2} \int_{0}^{1} \sum_{j=1}^{n} \sum_{i=1}^{n} \frac{\partial k_{j}}{\partial x_{i}}\left(\sigma_{1}, \sigma_{2}\right) \frac{\partial \sigma_{1}^{j}}{\partial s} \frac{\delta \sigma_{1}^{i}}{\delta x}-\frac{1}{2} \int_{0}^{1} \sum_{i=1}^{n} \frac{\partial k_{i}}{\partial t}\left(\sigma_{1}, \sigma_{2}\right) \frac{\partial \sigma_{1}^{i}}{\partial s} \frac{\delta \sigma_{2}}{\delta x} \\
& +\frac{1}{2} \int_{0}^{1} \sum_{i=1}^{n}\left[\sum_{j=1}^{n} \frac{\partial k_{i}}{\partial x_{j}}\left(\sigma_{1}, \sigma_{2}\right) \frac{\partial \sigma_{1}^{j}}{\partial s}+\frac{\partial k_{i}}{\partial t}\left(\sigma_{1}, \sigma_{2}\right) \frac{\partial \sigma_{2}}{\partial s}\right] \frac{\delta \sigma_{1}^{i}}{\delta x} \\
& -\left.\frac{1}{2} \sum_{i=1}^{n} k_{i}\left(\sigma_{1}, \sigma_{2}\right) \frac{\delta \sigma_{1}^{i}}{\delta x}\right|_{s=0} ^{s=1}+\int_{0}^{1} \sum_{i=1}^{n} \frac{\partial F}{\partial x_{i}}\left(\sigma_{1}, \sigma_{2}\right) \frac{\partial \sigma_{2}}{\partial s} \frac{\delta \sigma_{1}^{i}}{\delta x}
\end{aligned}
$$




$$
\begin{aligned}
& +\int_{0}^{1} \frac{\partial F}{\partial t}\left(\sigma, \sigma_{2}\right) \frac{\partial \sigma_{2}}{\partial s} \frac{\delta \sigma_{2}}{\delta x} \\
& -\int_{0}^{1} \sum_{i=1}^{n}\left[\frac{\partial F}{\partial t}\left(\sigma_{1}, \sigma_{2}\right) \frac{\delta \sigma_{2}}{\delta x}+\frac{\partial F}{\partial t}\left(\sigma_{1}, \sigma_{2}\right) \frac{\partial \sigma_{2}}{\partial x}\right] \frac{\delta \sigma_{2}}{\delta s}+\left.F\left(\sigma_{1}, \sigma_{2}\right) \frac{\delta \sigma_{2}}{\delta x}\right|_{s=0} ^{s=1} \\
& =\int_{0}^{1} \sum_{i=1}^{n}\left\{-\frac{1}{2} \frac{d}{d s}\left[\frac{\frac{d \sigma_{1}^{i}}{d s}(s)}{\frac{d \sigma_{2}}{d s}(s)+\varepsilon}\right]\right. \\
& +\frac{1}{2}\left(\sum_{j=1}^{n} \frac{\partial k_{i}}{\partial x_{j}}\left(\sigma_{1}(s), \sigma_{2}(s)\right) \frac{d \sigma_{1}^{j}}{d s}(s)+\frac{\partial k_{i}}{\partial t}\left(\sigma_{1}(s), \sigma_{2}(s)\right) \frac{\partial \sigma_{2}}{\partial s}(s)\right) \\
& \left.-\frac{1}{2} \sum_{j=1}^{n} \frac{\partial k_{j}}{\partial x_{i}}\left(\sigma_{1}(s), \sigma_{2}(s)\right) \frac{\partial \sigma_{1}^{j}}{\partial s}(s)+\frac{\partial F}{\partial x_{i}}\left(\sigma_{1}(s), \sigma_{2}(s)\right) \frac{\partial \sigma_{2}}{\partial s}(s)\right\} \frac{\delta \sigma_{1}^{i}}{\delta x} \\
& +\int_{0}^{1}\left\{\frac{1}{4} \frac{d}{d s}\left[\frac{\left|\frac{d \sigma_{1}}{d s}(s)\right|^{2}}{\left(\frac{d \sigma_{2}}{d s}(s)+\varepsilon\right)^{2}}\right]-\frac{1}{2} \sum_{i=1}^{n} \frac{\partial k_{i}}{\partial t}\left(\sigma_{1}(s), \sigma_{2}(s)\right) \frac{d \sigma_{1}^{i}}{d s}(s)-\right. \\
& \sum_{i=1}^{n} \frac{\partial F}{\partial x_{i}}\left(\sigma_{1}(s), \sigma_{2}(s) \frac{d \sigma_{1}^{i}}{d s}(s)\right\} \frac{\delta \sigma_{2}}{\delta x} \\
& +\left.\frac{1}{2} \sum_{i=1}^{n} \frac{\frac{d \sigma_{1}^{i}}{d s}(s)}{\frac{d \sigma_{2}}{d s}(s)+\varepsilon} \frac{\delta \sigma_{1}^{i}}{\delta x}\right|_{s=0} ^{s=1}-\left.\frac{1}{4} \frac{\left|\frac{d \sigma_{1}}{d s}(s)\right|^{2}}{\left(\frac{d \sigma_{2}}{d s}(s)+\varepsilon\right)^{2}} \frac{\delta \sigma_{1}^{i}}{\delta x}\right|_{s=0} ^{s=1} \\
& -\left.\frac{1}{2} \sum_{i=1}^{n} k_{i}\left(\sigma_{1}(s), \sigma_{2}(s)\right) \frac{\delta \sigma_{1}^{i}}{\delta x}\right|_{s=0} ^{s=1}+\left.F\left(\sigma_{1}(s), \sigma_{2}(s)\right) \frac{\delta \sigma_{2}}{\delta x}\right|_{s=0} ^{s=1} \\
& =\left.\frac{1}{2} \sum_{i=1}^{n} \frac{\frac{d \sigma_{1}^{i}}{d s}(s)}{\frac{d \sigma_{2}}{d s}(s)+\varepsilon} \frac{\delta \sigma_{1}^{i}}{\delta x}\right|_{s=0} ^{s=1}-\left.\frac{1}{4} \frac{\left|\frac{d \sigma_{1}}{d s}(s)\right|^{2}}{\left(\frac{d \sigma_{2}}{d s}(s)+\varepsilon\right)^{2}} \frac{\delta \sigma_{2}}{\delta x}\right|_{s=0} ^{s=1} \\
& -\left.\frac{1}{2} \sum_{i=1}^{n} k_{i}\left(\sigma_{1}(s), \sigma_{2}(s)\right) \frac{\delta \sigma_{1}^{i}}{\delta x}\right|_{s=0} ^{s=1}+\left.F\left(\sigma_{1}(s), \sigma_{2}(s)\right) \frac{\delta \sigma_{2}}{\delta x}\right|_{s=0} ^{s=1}
\end{aligned}
$$

because of (1.43) and (1.44). Observe that we do not move $t$ in calculating $\frac{\delta d_{\varepsilon}}{\delta x}(x, t)$. Hence $\frac{\delta \sigma_{2}}{\delta x}=0$ and we obtain

$$
\begin{aligned}
\frac{\delta d_{\varepsilon}}{\delta x}(x, t) & =\left.\frac{1}{2} \sum_{i=1}^{n} \frac{\frac{d \sigma_{1}^{i}}{d s}}{\frac{d \sigma_{2}}{d s}+\varepsilon} \frac{\delta \sigma_{1}^{i}}{\delta x}\right|_{s=0} ^{s=1}-\left.\frac{1}{2} \sum_{i=1}^{n} k_{i}\left(\sigma_{1}, \sigma_{2}\right)\left(\sigma_{1}, \sigma_{2}\right) \frac{\delta \sigma_{1}^{i}}{\delta x}\right|_{s=0} ^{s=1} \\
& =\frac{1}{2} \sum_{i=1}^{n} \frac{\frac{d \sigma_{1}^{i}}{d s}(1)}{\frac{d \sigma_{2}}{d s}(1)+\varepsilon} \frac{\delta \sigma_{1}^{i}}{\delta x}(1)-\frac{1}{2} \sum_{i=1}^{n} k_{i}\left(\sigma_{1}(1), \sigma_{2}(1)\right) \frac{\delta \sigma_{1}^{i}}{\delta x}(1)
\end{aligned}
$$

It follows that

$$
\nabla d_{\varepsilon}(x, t)=\frac{\delta d_{\varepsilon}}{\delta x}(x, t)=\frac{1}{2} \frac{\dot{\sigma}_{1}(1)}{\frac{\partial \sigma_{2}}{\partial s}(1)+\varepsilon}-\frac{1}{2} k(x, t)
$$

Similar calculation as above will show that

$$
\frac{\delta d_{\varepsilon}}{\delta t}(x, t)=\left.\frac{1}{2} \sum_{i=1}^{n} \frac{\left|\frac{d \sigma_{1}^{i}}{d s}(s)\right|^{2}}{\left(\frac{d \sigma_{2}}{d s}(s)+\varepsilon\right)^{2}} \frac{\delta \sigma_{1}^{i}}{\delta t}\right|_{s=0} ^{s=1}-\left.\frac{1}{4} \frac{\left|\frac{d \sigma_{1}}{d s}(s)\right|^{2}}{\left(\frac{d \sigma_{2}}{d s}(s)+\varepsilon\right)^{2}} \frac{\delta \sigma_{2}}{\delta t}\right|_{s=0} ^{s=1}
$$




$$
-\left.\frac{1}{2} \sum_{i=1}^{n} k_{i}\left(\sigma_{1}(s), \sigma_{2}(s)\right) \frac{\delta \sigma_{1}^{i}}{\delta t}\right|_{s=0} ^{s=1}+\left.F\left(\sigma_{1}(s), \sigma_{2}(s)\right) \frac{\delta \sigma_{2}}{\delta t}\right|_{s=0} ^{s=1}
$$

Observe that we do not move $x$ in calculating $\frac{\delta d_{\varepsilon}}{\delta t}(x, t)$. Hence $\frac{\delta \sigma_{1}^{i}}{\delta t}=0$ for $1 \leq i \leq n$ and we obtain (1.41). Equation (1.42) follows immediately from (1.39), (1.40) and (1.41).

Notice that $\nabla d_{\varepsilon}$ exists only almost everywhere as only for almost every $(x, t)$ there is a unique minimal curve $\sigma$ joining $(x, t)$ to $\left(x_{0}, t_{0}\right)$. Hence $(1.39),(1.40),(1.41)$ and (1.42) hold almost everywhere.

Corollary 1.1. In Theorem 1.2, let $k_{i}(x, t)=-f_{i}(x, t)$ and $F(x, t)=$ $\frac{1}{2} \sum_{n=1}^{n} \frac{\partial f_{i}}{\partial x_{i}}(x, t)+\frac{1}{4}|f(x, t)|^{2}+V(x, t)$. Let $g_{\varepsilon}(x, t)=2 d_{\varepsilon}(x, t)$. Then $g(x, t)$ satisfies condition (i) in Theorem 1.1.

Corollary 1.2. In Theorem 1.2, let $k_{i}(x, t)=2 f_{i}(x, t)$ and $F(x, t)=2 V(x, t)-$ $|\nabla V(x, t)|^{2}$. Let $h_{\varepsilon}(x, t)=d_{\varepsilon}(x, t)$. Then $h_{\varepsilon}(x, t)$ satisfies condition (ii) in Theorem 1.1 .

Corollary 1.3. In Theorem 1.2, let $k_{i}(x, t)=f_{i}(x, t)$ and $F(x, t)=\frac{1}{4}|f|^{2}-$ $\frac{2 \varepsilon_{1}^{3}+1}{2 \varepsilon_{1}^{2}} \sum_{i=1}^{n} \frac{\partial f_{i}}{\partial x_{i}}$. Let $\rho_{\varepsilon}(x, t)=\frac{2 \varepsilon_{1}^{2}}{2 \varepsilon_{1}^{3}+1} d_{\varepsilon}(s, t)$. Then $\rho_{\varepsilon}(x, t)$ satisfies condition (iii) in Theorem 1.1 .

REMARK. Notice the difference between $\varepsilon_{1}$ in Theorem 1.1 and $\varepsilon$ in Theorem 1.2.

Lemma 1.1. Let $c \geq 4$ be a constant. Assume that

$$
\begin{aligned}
|f(x, t)| & \leq c(1+|x|) \\
|\nabla f(x, t)| & =\sqrt{\sum_{i=1}^{n}\left|\nabla f_{i}\right|^{2}} \leq c(1+|x|) \\
|V(x, t)| & \leq c\left(1+|x|^{2}\right) \\
|\nabla V(x, t)| & \leq c(1+|x|)
\end{aligned}
$$

Then the following statements holds.

(a) $g_{\varepsilon}(x, t)$ in Corollary 1.1 has lower bound in terms of

$$
\left[\frac{1}{2\left(t-t_{0}+\varepsilon\right)}-\frac{3 c^{2}\left(\pi^{2}+1\right)}{\pi^{2}}\left(t-t_{0}\right)-\frac{c\left(5 \pi^{2}+3\right)}{4 \pi^{2}}\right] x^{2}+\text { lower order terms in } x .
$$

(b) $h_{\varepsilon}(x, t)$ in Corollary 1.2 has lower bound in terms of

$$
\left(\frac{1}{4\left(t-t_{0}+\varepsilon\right)}-\frac{c\left(5 \pi^{2}+3\right)}{4 \pi^{2}}-\frac{3 c^{2}\left(t-t_{0}\right)\left(\pi^{2}+1\right)}{\pi^{2}}\right) x^{2}+\text { lower order terms in } x .
$$

(c) $\rho_{\varepsilon}(x, t)$ in Corollary 1.3 has linear bound in terms of $\frac{2 \varepsilon_{1}^{2}}{2 \varepsilon_{1}^{3}+1}\left[\left(\frac{1}{4\left(t-t_{0}+\varepsilon\right)}-\right.\right.$ $\left.\left(t-t_{0}\right) c\left(\frac{c}{16}+\frac{2 \varepsilon_{1}^{3}+1}{8 \varepsilon_{1}^{2}}\right)-\frac{3 c}{4}-\frac{3 c}{8 \pi^{2}}-\frac{3 c\left(\pi^{2}+1\right)}{8 \pi^{2}}\left(t-t_{0}\right)\left(\frac{c}{2}+\frac{2 \varepsilon_{1}^{3}+1}{\varepsilon_{1}^{2}}\right)\right] x^{2}+$ lower order terms in $x$. 
Proof.

(a) $\quad|F(x, t)|=\left.\left|\frac{1}{2} \sum_{i=1}^{n} \frac{\partial f_{i}}{\partial x_{i}}(x, t)+\frac{1}{4}\right| f(x, t)\right|^{2}+V(x, t) \mid$

$$
\begin{aligned}
& \leq \frac{1}{2} \sum_{i=1}^{n}\left|\frac{\partial f_{i}}{\partial x_{i}}\right|+\frac{1}{4}|f(x, t)|^{2}+|V(x, t)| \\
& \leq \frac{1}{2} c(1+|x|)+\frac{c^{2}}{4}(1+|x|)^{2}+c\left(1+|x|^{2}\right) \\
& \leq c^{2}(1+|x|)^{2}
\end{aligned}
$$

Recall that $\mathcal{P}$ consists of path $\sigma=\left(\sigma_{1}, \sigma_{2}\right)$ such that

$$
\begin{aligned}
& \sigma(0)=\left(x_{0}, t_{0}\right), \sigma(1)=(x, t), \sigma_{2}(s)=\left(t-t_{0}\right) s+t_{0}, \int_{0}^{1} \sigma_{1}(s) d s=\frac{x+x_{0}}{2} \\
& g_{\varepsilon}(x, t)=2 d_{\varepsilon}(x, t)=2 \min _{\sigma \in \mathcal{P}} E_{\varepsilon}(\sigma) \\
& =\min _{\sigma \in \mathcal{P}}\left\{\frac{1}{2} \int_{0}^{1} \frac{|\dot{\sigma}(s)|^{2}}{\frac{\partial \sigma_{2}}{d s}+\varepsilon}-\int_{0}^{1} \sum_{i=1}^{n} k_{i}\left(\sigma_{1}(s), \sigma_{2}(s)\right) \frac{d \sigma_{1}^{i}}{d s}+2 \int_{0}^{1} F\left(\sigma_{1}(s), \sigma_{2}(s)\right) \frac{d \sigma_{2}}{d s}\right\} \\
& =\min _{\sigma \in \mathcal{P}}\left\{\frac{1}{2} \int_{0}^{1} \frac{|\dot{\sigma}(s)|^{2}}{t-t_{0}+\varepsilon}+\int_{0}^{1} \sum_{i=1}^{n} f_{i}\left(\sigma_{1}(s), \sigma_{2}(s)\right) \frac{d \sigma_{1}^{i}}{d s}+2 \int_{0}^{1} F\left(\sigma_{1}(s), \sigma_{2}(s)\right) \frac{d \sigma_{2}}{d s}\right\} \\
& \geq \min _{\sigma \in \mathcal{P}}\left\{\frac{1}{2\left(t-t_{0}+\varepsilon\right)} \int_{0}^{1}\left|\dot{\sigma}_{1}(s)\right|^{2}-\int_{0}^{1}\left|f\left(\sigma_{1}(s), \sigma_{2}(s)\right)\right|\left|\frac{d \sigma_{1}}{d s}\right|\right. \\
& \left.-2 \int_{0}^{1} c^{2}\left(1+\left|\sigma_{1}(s)\right|\right)^{2} \frac{d \sigma_{2}}{d s}\right\} \\
& \geq \min _{\sigma \in \mathcal{P}}\left\{\frac{1}{2\left(t-t_{0}+\varepsilon\right)} \int_{0}^{1}\left|\dot{\sigma}_{1}(s)\right|^{2}-\int_{0}^{1} c\left(1+\left|\sigma_{1}(s)\right|\right)\left|\frac{d \sigma_{1}}{d s}\right|\right. \\
& \left.-2\left(t-t_{0}\right) \int_{0}^{1} c^{2}\left(1+\left|\sigma_{1}(s)\right|\right)^{2}\right\} \\
& \geq \min _{\sigma \in \mathcal{P}}\left\{\left(\frac{1}{2\left(t-t_{0}+\varepsilon\right)}-\frac{c}{2}\right) \int_{0}^{1}\left|\dot{\sigma}_{1}(s)\right|^{2}-\left(2\left(t-t_{0}\right) c^{2}+\frac{c}{2}\right) \int_{0}^{1}\left(1+\left|\sigma_{1}(s)\right|\right)^{2}\right\} \\
& =\min _{\sigma \in \mathcal{P}}\left\{\left(\frac{1}{2\left(t-t_{0}+\varepsilon\right)}-\frac{c}{2}\right) \int_{0}^{1}\left|\dot{\sigma}_{1}(s)\right|^{2}-\left(2\left(t-t_{0}\right) c^{2}+\frac{c}{2}\right)\right. \\
& \left.-\left(4\left(t-t_{0}\right) c^{2}+c\right) \int_{0}^{1}\left|\sigma_{1}(s)\right|-\left(2\left(t-t_{0}\right) c^{2}+\frac{c}{2}\right) \int_{0}^{1}\left|\sigma_{1}(s)\right|^{2}\right\} \\
& \geq \min _{\sigma \in \mathcal{P}}\left\{\left(\frac{1}{2\left(t-t_{0}+\varepsilon\right)}-\frac{c}{2}\right) \int_{0}^{1}\left|\dot{\sigma}_{1}(s)\right|^{2}-\left(2\left(t-t_{0}\right) c^{2}+\frac{c}{2}\right)\right. \\
& \left.-\left[6\left(t-t_{0}\right) c^{2}+\frac{3}{2} c\right] \int_{0}^{1}\left(1+\left|\sigma_{1}(s)\right|\right)^{2}\right\}
\end{aligned}
$$

Recall that the Poincare inequality states that

$$
\int_{0}^{1}\left|\sigma_{1}(s)-\int_{0}^{1} \sigma_{1}(s) d s\right|^{2} \leq D \int_{0}^{1}\left|\dot{\sigma}_{1}\right|^{2}, \text { where } D=\frac{1}{4 \pi^{2}}
$$


Since

$$
\left|\sigma_{1}(s)\right|^{2} \leq 2\left|\sigma_{1}(s)-\int_{0}^{1} \sigma_{1}\right|^{2}+2\left(\int_{0}^{1} \sigma_{1}\right)^{2}
$$

we have

$$
\begin{aligned}
\int_{0}^{1}\left|\sigma_{1}(s)\right|^{2} & \leq 2 \int_{0}^{1}\left|\sigma_{1}(s)-\int_{0}^{1} \sigma_{1}\right|^{2}+2\left(\int_{0}^{1} \sigma_{1}\right)^{2} \\
& \leq 2 D \int_{0}^{1}\left|\dot{\sigma}_{1}\right|^{2}+2\left(\int_{0}^{1} \sigma_{1}\right)^{2}
\end{aligned}
$$

Hence

$$
\begin{aligned}
g_{\varepsilon}(x, t) & \geq \min _{\sigma \in \mathcal{P}}\left\{\left[\frac{1}{\left.2\left(t-t_{0}\right)+\varepsilon\right)}-\frac{c}{2}-\left(6\left(t-t_{0}\right) c^{2}+\frac{3}{2} c\right) 2 D\right] \int_{0}^{1}|\dot{\sigma}(s)|^{2}\right. \\
& \left.-\left(2\left(t-t_{0}\right) c^{2}+\frac{c}{2}\right)-\left(12\left(t-t_{0}\right) c^{2}+3 c\right)\left(\int_{0}^{1} \sigma_{1}\right)^{2}\right\}
\end{aligned}
$$

Observe that $\int_{0}^{1}\left|\dot{\sigma}_{1}\right|^{2} \geq\left|\int_{0}^{1} \dot{\sigma}_{1}\right|^{2}=\left|x-x_{0}\right|^{2}$ by Schwartz inequality. It follows that

$$
\begin{aligned}
g_{\varepsilon}(x, t) \geq & {\left[\frac{1}{2\left(t-t_{0}+\varepsilon\right)}-\frac{c}{2}-\left(12\left(t-t_{0}\right) c^{2}+3 c\right) D\right]\left|x-x_{0}\right|^{2} } \\
& -\left(2\left(t-t_{0}\right) c^{2}+\frac{c}{2}\right)-\left(12\left(t-t_{0}\right) c^{2}+3 c\right)\left|\frac{x+x_{0}}{2}\right|^{2} \\
= & {\left[\frac{1}{2\left(t-t_{0}+\varepsilon\right)}-\frac{c}{2}-12\left(t-t_{0}\right) c^{2} D-3 c D-3\left(t-t_{0}\right) c^{2}-\frac{3}{4} c\right] x^{2} } \\
& \quad+\text { lower order terms in } x . \\
= & {\left[\frac{1}{2\left(t-t_{0}+\varepsilon\right)}-\frac{3 c^{2}\left(\pi^{2}+1\right)}{\pi^{2}}\left(t-t_{0}\right)-\frac{c\left(5 \pi^{2}+3\right)}{4 \pi^{2}}\right] x^{2} } \\
& \quad+\text { lower order terms in } x .
\end{aligned}
$$

(b)

$$
\begin{aligned}
\mid F(x, t) & =|2 V(x, t)-| \nabla V(x, t)|| \\
& \leq 2|V(x, t)|+|\nabla V(x, t)| \\
& \leq 2 c\left(1+|x|^{2}\right)+c(1+|x|) \\
& \leq\left(c^{2}+2 c\right)(1+|x|)^{2} \\
& =2 c^{2}(1+|x|)^{2}
\end{aligned}
$$

$$
\begin{gathered}
h_{\varepsilon}(x, t)=d_{\varepsilon}(x, t)=\min _{\sigma \in \mathcal{P}} E_{\varepsilon}(\sigma) \\
=\min _{\sigma \in \mathcal{P}}\left\{\frac{1}{4} \int_{0}^{1} \frac{\left|\dot{\sigma}_{1}(s)\right|^{2}}{\frac{\partial \sigma_{2}}{\partial s}+\varepsilon}-\frac{1}{2} \int_{0}^{1} \sum_{i=1}^{n} k_{i}\left(\sigma_{1}(s), \sigma_{2}(s)\right) \frac{d \sigma_{1}^{i}}{d s}+\int_{0}^{1} F\left(\sigma_{1}(s), \sigma_{2}(s)\right) \frac{d \sigma_{2}}{d s}\right\} \\
\geq \min _{\sigma \in \mathcal{P}}\left\{\frac{1}{4\left(t-t_{0}+\varepsilon\right)} \int_{0}^{1}\left|\dot{\sigma}_{1}(s)\right|^{2}-\int_{0}^{1} c\left(1+\left|\sigma_{1}(s)\right|\right)\left|\frac{d \sigma_{1}}{d s}\right|\right. \\
\left.-\left(t-t_{0}\right) \int_{0}^{1} 2 c^{2}\left(1+\left|\sigma_{1}(s)\right|\right)^{2}\right\}
\end{gathered}
$$




$$
\begin{aligned}
& \geq \min _{\sigma \in \mathcal{P}}\left\{\left(\frac{1}{4\left(t-t_{0}+\varepsilon\right)}-\frac{c}{2}\right) \int_{0}^{1}\left|\dot{\sigma}_{1}(s)\right|^{2}-\left(2\left(t-t_{0}\right) c^{2}+\frac{c}{2}\right) \int_{0}^{1}\left(1+\left|\sigma_{1}(s)\right|\right)^{2}\right\} \\
& =\min _{\sigma \in \mathcal{P}}\left\{\left(\frac{1}{4\left(t-t_{0}+\varepsilon\right)}-\frac{c}{2}\right) \int_{0}^{1}\left|\dot{\sigma}_{1}(s)\right|^{2}-\left(2\left(t-t_{0}\right) c^{2}+\frac{c}{2}\right)\right. \\
& \left.-\left(4\left(t-t_{0}\right) c^{2}+c\right) \int_{0}^{1}\left|\sigma_{1}(s)\right|-\left(2\left(t-t_{0}\right) c^{2}+\frac{c}{2}\right) \int_{0}^{1}\left|\sigma_{1}(s)\right|^{2}\right\} \\
& \geq \min _{\sigma \in \mathcal{P}}\left\{\left(\frac{1}{4\left(t-t_{0}+\varepsilon\right)}\right)-\frac{c}{2}\right) \int_{0}^{1}\left|\dot{\sigma}_{1}(s)\right|^{2}-\left(2\left(t-t_{0}\right) c^{2}+\frac{c}{2}\right) \\
& \left.-\left(6\left(t-t_{0}\right) c^{2}+\frac{3 c}{2}\right) \int_{0}^{1}\left|\sigma_{1}(s)\right|^{2}\right\} \\
& \geq \min _{\sigma \in \mathcal{P}}\left\{\left[\frac{1}{4\left(t-t_{0}+\varepsilon\right)}-\frac{c}{2}-\left(12\left(t-t_{0}\right) c^{2} D+3 c D\right)\right] \int_{0}^{1}\left|\dot{\sigma}_{1}(s)\right|^{2}\right. \\
& \left.-\left(2\left(t-t_{0}\right) c^{2}+\frac{c}{2}\right)-\left(12\left(t-t_{0}\right) c^{2}+3 c\right)\left(\int_{0}^{1} \sigma_{1}\right)^{2}\right\} \\
& \geq \min _{\sigma \in \mathcal{P}}\left\{\left[\frac{1}{4\left(t-t_{0}+\varepsilon\right)}-\frac{c}{2}-3 c D-12\left(t-t_{0}\right) c^{2} D\right]\left|\int_{0}^{1} \dot{\sigma}_{1}\right|^{2}\right. \\
& \left.-\left(2\left(t-t_{0}\right) c^{2}+\frac{c}{2}\right)-\left(12\left(t-t_{0}\right) c^{2}+3 c\right)\left(\int_{0}^{1} \sigma_{1}\right)^{2}\right\} \\
& =\left(\frac{1}{4\left(t-t_{0}+\varepsilon\right)}-\frac{c\left(2 \pi^{2}+3\right)}{4 \pi^{2}}-\frac{3 c^{2}\left(t-t_{0}\right)}{\pi^{2}}\right)\left|x-x_{0}\right|^{2} \\
& \left(2\left(t-t_{0}\right) c^{2}+\frac{c}{2}\right)-\left(12\left(t-t_{0}\right) c^{2}+3 c\right)\left|\frac{x+x_{0}}{2}\right|^{2} \\
& =\left(\frac{1}{4\left(t-t_{0}+\varepsilon\right)}-\frac{c\left(5 \pi^{2}+3\right)}{4 \pi^{2}}-\frac{3 c^{2}\left(t-t_{0}\right)\left(\pi^{2}+1\right)}{\pi^{2}}\right) x^{2}+\text { lower order terms in } x \text {. } \\
& \text { (c) }|F(x, t)|=\left.\left|\frac{1}{4}\right| f\right|^{2}-\frac{2 \varepsilon_{1}^{3}+1}{\partial \varepsilon_{1}^{2}} \sum_{i=1}^{n} \frac{\partial f_{i}}{\partial x_{i}} \mid \\
& \leq \frac{1}{4}|f|^{2}+\frac{2 \varepsilon_{1}^{3}+1}{2 \varepsilon_{1}^{2}}\left|\sum_{i=1}^{n} \frac{\partial f_{i}}{\partial x_{i}}\right| \\
& \leq \frac{1}{4} c^{2}(1+|x|)^{2}+\frac{2 \varepsilon^{3}+1}{2 \varepsilon^{2}} c(1+|x|) \\
& \leq c\left(\frac{c}{4}+\frac{2 \varepsilon_{1}^{3}+1}{2 \varepsilon_{1}^{2}}\right)(1+|x|)^{2} \\
& \rho_{\varepsilon}(x, t)=\frac{2 \varepsilon_{1}^{2}}{2 \varepsilon_{1}^{3}+1} d_{\varepsilon}(x, t) \\
& =\frac{2 \varepsilon_{1}^{2}}{2 \varepsilon_{1}^{3}+1} \min _{\sigma \in \mathcal{P}}\left\{\frac{1}{4} \int_{0}^{1} \frac{\left|\dot{\sigma}_{1}(s)\right|^{2}}{\frac{\partial \sigma_{2}}{\partial s}+\varepsilon}-\frac{1}{2} \int_{0}^{1} \sum_{i=1}^{n} k_{i}\left(\sigma_{1}(s), \sigma_{2}(s)\right) \frac{d \sigma_{1}^{i}}{d s}\right. \\
& +\int_{0}^{1} F\left(\sigma_{1}(s), \sigma_{2}(s) \frac{d \sigma_{2}}{d s}\right\} \\
& \geq \frac{2 \varepsilon_{1}^{2}}{2 \varepsilon_{1}^{3}+1} \min _{\sigma \in \mathcal{P}}\left\{\frac{1}{4\left(t-t_{0}+\varepsilon\right)} \int_{0}^{1}\left|\dot{\sigma}_{1}(s)\right|^{2}-\frac{1}{2} \int_{0}^{1} c\left(1+\left|\sigma_{1}(s)\right|\right)\left|\frac{d \sigma_{1}}{d s}\right|\right.
\end{aligned}
$$




$$
\begin{aligned}
& -\left(t-t_{0}\right) \int_{0}^{1} c\left(\frac{c}{4}+\frac{2 \varepsilon_{1}^{3}+1}{2 \varepsilon_{1}^{2}}\right)\left(1+\sigma_{1}(s) \mid\right)^{2} \\
& \geq \frac{2 \varepsilon_{1}^{2}}{2 \varepsilon_{1}^{3}+1} \min _{\sigma \in \mathcal{P}}\left\{\left(\frac{1}{4\left(t-t_{0}+\varepsilon\right)}-\frac{c}{4}\right) \int_{0}^{1}\left|\dot{\sigma}_{1}(s)\right|^{2}-\left[\left(t-t_{0}\right) c\left(\frac{c}{4}+\frac{2 \varepsilon_{1}^{3}+1}{2 \varepsilon_{1}^{2}}\right)+\frac{c}{2}\right]\right. \\
& \left.\int_{0}^{1}\left(1+\left|\sigma_{1}(s)\right|\right)^{2}\right\} \\
& =\frac{2 \varepsilon_{1}^{2}}{2 \varepsilon_{1}^{3}+1} \min _{\sigma \in \mathcal{P}}\left\{\left(\frac{1}{4\left(t-t_{0}+\varepsilon\right)}-\frac{c}{4}\right) \int_{0}^{1}\left|\dot{\sigma}_{1}(s)\right|^{2}-\left[\left(t-t_{0}\right) c\left(\frac{c}{4}+\frac{2 \varepsilon_{1}^{3}+1}{2 \varepsilon_{1}^{2}}\right)+\frac{c}{2}\right]\right. \\
& -\left[\left(t-t_{0}\right) c\left(\frac{c}{4}+\frac{2 \varepsilon_{1}^{3}+1}{2 \varepsilon_{1}^{2}}\right)+c\right] \int_{0}^{1}\left|\dot{\sigma}_{1}(s)\right| \\
& -\left[\left[\left(t-t_{0}\right) c\left(\frac{c}{4}+\frac{2 \varepsilon_{1}^{3}+1}{2 \varepsilon_{1}^{2}}\right)+\frac{c}{2}\right] \int_{0}^{1}\left|\sigma_{1}(s)\right|^{2}\right. \\
& \geq \frac{2 \varepsilon_{1}^{2}}{2 \varepsilon_{1}^{3}+1} \min _{\sigma \in \mathcal{P}}\left\{\left(\frac{1}{4\left(t-t_{0}+\varepsilon\right)}-\frac{c}{4}\right) \int_{0}^{1}\left|\dot{\sigma}_{1}(s)\right|^{2}-\left[\left(t-t_{0}\right) c\left(\frac{c}{4}+\frac{2 \varepsilon_{1}^{3}+1}{2 \varepsilon_{1}^{2}}\right)+\frac{c}{2}\right]\right\} \\
& \left.-\left[\left(t-t_{0}\right) c\left(\frac{3 c}{4}+\frac{3\left(2 \varepsilon_{1}^{3}+1\right)}{2 \varepsilon_{1}^{2}}\right)+\frac{3 c}{2}\right] \int_{0}^{1}\left|\sigma_{1}(s)\right|^{2}\right\} \\
& \geq \frac{2 \varepsilon_{1}^{2}}{2 \varepsilon_{1}^{3}+1} \min _{\sigma \in \mathcal{P}}\left\{\left(\frac{1}{4\left(t-t_{0}+\varepsilon\right)}-\frac{c}{4}-\frac{3 c D}{2}\left(\left(t-t_{0}\right)\left(\frac{c}{2}+\frac{2 \varepsilon_{1}^{3}+1}{\varepsilon_{1}^{2}}\right)+1\right)\right] \int_{0}^{1}\left|\dot{\sigma}_{1}(s)\right|^{2}\right. \\
& -\left[\left(t-t_{0}\right) c\left(\frac{c}{4}+\frac{2 \varepsilon_{1}^{3}+1}{2 \varepsilon_{1}^{2}}\right)+\frac{c}{2}\right]-\frac{3 c}{2}\left[\left(t-t_{0}\right)\left(\frac{c}{2}+\frac{2 \varepsilon_{1}^{3}+1}{\varepsilon_{1}^{2}}\right)+1\right]\left|\int_{0}^{1} \sigma_{1}(s)\right|^{2} \\
& \geq \frac{2 \varepsilon_{1}^{2}}{2 \varepsilon_{1}^{3}+1} \min _{\sigma \in \mathcal{P}}\left\{\left(\frac{1}{4\left(t-t_{0}+\varepsilon\right)}-\frac{c}{4}-\frac{3 c D}{2}\left(\left(t-t_{0}\right)\left(\frac{c}{2}+\frac{2 \varepsilon_{1}^{3}+1}{\varepsilon_{1}^{2}}\right)+1\right)\right] \int_{0}^{1}\left|\dot{\sigma}_{1}\right|^{2}\right. \\
& \left.-\left[\left(t-t_{0}\right) c\left(\frac{c}{4}+\frac{2 \varepsilon_{1}^{3}+1}{2 \varepsilon_{1}^{2}}\right)+\frac{c}{2}\right]-\frac{3 c}{2}\left[\left(t-t_{0}\right)\left(\frac{c}{2}+\frac{2 \varepsilon_{1}^{3}+1}{\varepsilon_{1}^{2}}\right)+1\right]\left|\int_{0}^{1} \sigma_{1}(s)\right|^{2}\right\} \\
& =\frac{2 \varepsilon_{1}^{2}}{2 \varepsilon_{1}^{3}+1}\left\{\left[\frac{1}{4\left(t-t_{0}+\varepsilon\right)}-\frac{c}{4}-\frac{3 c D}{2}\left(\left(t-t_{0}\right)\left(\frac{c}{2}+\frac{2 \varepsilon_{1}^{3}+1}{\varepsilon_{1}^{2}}\right)+1\right)\right]\left|x-x_{0}\right|^{2}\right. \\
& \left.-\left[\left(t-t_{0}\right) c\left(\frac{c}{4}+\frac{2 \varepsilon_{1}^{3}+1}{2 \varepsilon_{1}^{2}}\right)+\frac{c}{2}\right]-\frac{3 c}{2}\left[\left(t-t_{0}\right)\left(\frac{c}{2}+\frac{2 \varepsilon_{1}^{3}+1}{\varepsilon_{1}^{2}}\right)+1\right]\left|\frac{x+x_{0}}{2}\right|^{2}\right\} \\
& =\frac{2 \varepsilon_{1}^{2}}{2 \varepsilon_{1}^{3}+1}\left[\frac{1}{4\left(t-t_{0}+\varepsilon\right)}-\left(t-t_{0}\right) c\left(\frac{c}{16}+\frac{2 \varepsilon_{1}^{3}+1}{8 \varepsilon_{1}^{2}}\right)-\frac{3 c}{4}-\frac{3 c}{8 \pi^{2}}\right. \\
& \left.-\frac{3 c\left(\pi^{2}+1\right)}{8 \pi^{2}}\left(t-t_{0}\right)\left(\frac{c}{2}+\frac{2 \varepsilon_{1}^{3}+1}{\varepsilon_{1}^{2}}\right)\right] x^{2}+\text { lower order term in } x \text {. }
\end{aligned}
$$

In view of Lemma 1.1, we have the following Lemma 1.2

LEMMA 1.2. Assume that (1.44) - (1.47) hold i.e. $|f(x, t)| \leq c(1+|x|), \nabla f(x, t) \leq$ $c(1+|x|),|V(x, t)| \leq c\left(1+|x|^{2}\right)$ and $|\nabla V(x, t)| \leq c(|1+| x \mid)$, where $c \geq 4$ is a constant. Let $\tilde{c}$ be a constant strictly less than $\min \left(\frac{1}{4}, \frac{\varepsilon_{1}^{2}}{4 \varepsilon_{1}^{3}+2}\right)$. Let $\rho(x, t)=\frac{\overline{\widetilde{c}}\left(1+|x|^{2}\right)}{t-t_{0}+\varepsilon}$. Then the conditions (i), (ii) and (iii) in Theorem 1.1 are satisfied for $t-t_{0}+\varepsilon$ sufficiently small i.e.,

$$
\text { (i) } \frac{\partial \rho}{\partial t}+\frac{1}{2}|\nabla \rho|^{2}-\sum_{i=1}^{n} f_{i} \rho_{i}-\sum_{i=1}^{n} f_{i, i}-2 V \leq 0 \text { for } t-t_{0}+\varepsilon \text { sufficiently small. }
$$


(ii) $\frac{\partial \rho}{\partial t}+|\nabla \rho+f|^{2}-2 V+|\nabla V|^{2} \leq 0$ for $t-t_{0}+\varepsilon$ sufficiently small

(iii) $\frac{\partial \rho}{\partial t}+\left(\varepsilon_{1}+\frac{1}{2 \varepsilon_{1}^{2}}\right)|\nabla \rho|^{2}+\sum_{i=1}^{n} \rho_{i} f_{i}+\sum_{i=1}^{n} f_{i, i} \leq 0$ for $t-t_{0}+\varepsilon$ sufficiently small.

Proof.

$$
\frac{\partial \rho}{\partial t}=-\frac{\widetilde{c}\left(1+|x|^{2}\right)}{\left(t-t_{0}+\varepsilon\right)^{2}}, \quad \frac{\partial \rho}{\partial x_{i}}=\frac{2 \widetilde{c} x_{i}}{t-t_{o}+\varepsilon}, \quad|\nabla \rho|^{2}=\frac{4 \widetilde{c}^{2}|x|^{2}}{\left(t-t_{0}+\varepsilon\right)^{2}}
$$

(i)

$$
\begin{aligned}
& \frac{\partial \rho}{\partial t}+\frac{1}{2}|\nabla \rho|^{2}-\sum_{i=1}^{n} f_{i} \rho_{i}-\sum_{i=1}^{n} f_{i, i}-2 V \\
\leq & \frac{-\widetilde{c}\left(1+|x|^{2}\right)}{\left(t-t_{0}+\varepsilon\right)^{2}}+\frac{2 \widetilde{c}^{2}|x|^{2}}{\left(t-t_{0}+\varepsilon\right)^{2}}+c(1+|x|) \frac{2 \widetilde{c}|x|}{t-t_{0}+\varepsilon}+n c(1+|x|) \\
& +2 c(1+|x|)^{2} \\
\leq & \frac{\left(-\widetilde{c}+2 \widetilde{c}^{2}\right)}{\left(t-t_{0}+\varepsilon\right)^{2}}\left(1+|x|^{2}\right)+\frac{2 \widetilde{c} c}{t-t_{0}+\varepsilon}(1+|x|)^{2}+n c(1+|x|)^{2}+2 c(1+|x|)^{2} \\
\leq & \left(\frac{-\widetilde{c}(1-2 \widetilde{c})}{\left(t-t_{0}+\varepsilon\right)^{2}}+\frac{4 \widetilde{c} c}{t-t_{0}+\varepsilon}+2 n c+4 c\right)\left(1+|x|^{2}\right) \\
< & 0 \\
& \text { for } t-t_{0}+\varepsilon \text { sufficiently small because } 0<\widetilde{c}<\frac{1}{4} .
\end{aligned}
$$

(ii) $\frac{\partial \rho}{\partial t}+|\nabla \rho+f|^{2}-2 V+|\nabla V|^{2}$

$$
\begin{aligned}
& =\frac{\partial \rho}{\partial t}+|\nabla \rho|^{2}+2 \nabla \rho \cdot f+|f|^{2}-2 V+|\nabla V|^{2} \\
& \leq-\frac{\widetilde{c}\left(1+|x|^{2}\right)}{\left(t-t_{0}+\varepsilon\right)^{2}}+\frac{4 \widetilde{c}^{2}|x|^{2}}{\left(t-t_{0}+\varepsilon\right)^{2}}+\frac{4 \widetilde{c}|x|}{t-t_{0}+\varepsilon} c(1+|x|) \\
& \quad \quad+c^{2}\left(1+|x|^{2}\right)+2 c\left(1+|x|^{2}\right)+c^{2}(1+|x|)^{2}
\end{aligned}
$$$$
<0
$$

for $t-t_{0}+\varepsilon$ sufficiently small because $0<\widetilde{c}<\frac{1}{4}$.

(iii)

$$
\begin{aligned}
& \frac{\partial \rho}{\partial t}+\left(\varepsilon_{1}+\frac{1}{2 \varepsilon_{1}^{2}}\right)|\nabla \rho|^{2}+\sum_{i=1}^{n} \rho_{i} f_{i}+\sum_{i=1}^{n} f_{i, i} \\
\leq & \frac{-\widetilde{c}\left(1+|x|^{2}\right)}{\left(t-t_{0}+\varepsilon\right)^{2}}+\left(\varepsilon_{1}+\frac{1}{2 \varepsilon_{1}^{2}}\right) \frac{4 \widetilde{c}^{2}}{\left(t-t_{0}+\varepsilon\right)^{2}}|x|^{2}+\frac{2 \widetilde{c}|x|}{t-t_{0}+\varepsilon} c(1+|x|) \\
& \quad+c(1+|x|) \\
\leq & {\left[\frac{-\widetilde{c}\left(1-\left(4 \varepsilon_{1}+\frac{2}{\varepsilon_{1}^{2}}\right) \widetilde{c}\right)}{\left(t-t_{0}+\varepsilon\right)^{2}}+\frac{4 \widetilde{c} c}{t-t_{0}+\varepsilon}+2 c\right]\left(1+|x|^{2}\right) } \\
< & 0
\end{aligned}
$$

for $t-t_{0}+\varepsilon$ sufficiently small because $0<\tilde{c}<\frac{\varepsilon_{1}^{2}}{4 \varepsilon_{1}^{3}+2}$. 
Consider the parabolic differential equation

$$
\begin{aligned}
\frac{\partial u}{\partial t} & =\Delta u+\sum_{i=1}^{n} f_{i} \frac{\partial u}{\partial x_{i}}-V u \quad \text { on } \mathbb{R}^{n} \\
u(x, 0) & =\psi(x)
\end{aligned}
$$

where $f$ grows at most linearly and $V$ grows at most quadratically satisfying (1.44) (1.47) respectively. Let $f_{2 R}$ and $V_{2 R}$ be the functions obatined by multiplying $f$ and $V$ respectively by a cut off function $\sigma$ which is equal to one in the ball of radius $R \geq 1$ and equal to zero outside a ball of radius $2 R$. We can choose $\sigma$ such that

$$
|\nabla \sigma(x)| \leq \frac{4}{1+|x|} \quad \text { and } \quad|\triangle \sigma(x)| \leq \frac{4}{1+|x|^{2}}
$$

Consider the following equation

$$
\frac{\partial u_{2 R}}{\partial t}=\Delta u_{2_{R}}+\sum_{i=1}^{n}\left(f_{2 R}\right)_{i} \frac{\partial u_{2 R}}{\partial x_{i}}-V_{2 R} u_{2 R}
$$

in the ball $B_{2 R}$ of radius $2 R$ with the Neumann condition, where $\left(f_{2 R}\right)_{i}$ denotes the $i$-th component of $f_{2 R}$. Let $\psi_{2 R}=\psi(x) \sigma(x)$. Then the second initial-boundary problem

$$
\begin{cases}\frac{\partial u_{2 R}}{\partial t}=\Delta u_{2 R}+\sum_{i=1}^{n}\left(f_{2 R}\right)_{i} \frac{\partial u_{2 R}}{\partial x_{i}}-V_{2 R} u_{2 R} & \text { on } B_{2 R} \times(0, T] \\ u_{2 R}(x, 0)=\psi_{2 R}(x) & \text { on } \bar{B}_{2 R} \\ \frac{\partial u_{2 R}}{\partial \nu}=0 & \text { on } \partial B_{2 R} \times(0, T]\end{cases}
$$

has an unique solution (c.f. $[\mathrm{Fr}]$ p.144 Theorem 2) for $t \in[0, \infty)$.

LEMMA 1.3. Assume that (1.44) - (1.47) hold, i.e., $|f(x, t)| \leq c(1+|x|)$, $|\nabla f(x, t) \leq c(1+|x|)| V,(x, t) \mid \leq c\left(1+|x|^{2}\right)$ and $|\nabla V(x, t)| \leq c(1+|x|)$ where $c \geq 4$ is a constant. Let $\tilde{c}<\min \left(\frac{1}{4}, \frac{\varepsilon_{1}^{2}}{4 \varepsilon_{1}^{3}+2}\right)$ be a constant. Choose $T$ and $\varepsilon$ suitably small so that conclusions (i), (ii) and (iii) in Lemma 1.2 hold for $\rho(x, t)=\frac{\widetilde{c}\left(1+|x|^{2}\right)}{t+\varepsilon}$ for all $0 \leq t \leq T$. Then for $0 \leq t \leq T$

$$
\begin{aligned}
& \text { (i) } \int_{B_{2 R} \times\{t\}} e^{\rho} u_{2 R}^{2} \leq \int_{B_{2 R} \times\{0\}} e^{\rho} u_{2 R}^{2} \\
& \text { (ii) } \int_{B_{2 R} \times\{t\}} e^{\rho}\left|\nabla u_{2 R}\right|^{2} \leq \int_{B_{2 R} \times\{0\}} e^{\rho}\left(\nabla u_{2_{R}}\right)^{2}+\int_{0}^{t} \int_{B_{2 R}} e^{\rho(x, s)}\left(u_{2 R}(x, s)\right)^{2} \\
& \text { (iii) } \int_{B_{2 R} \times\{t\}} e^{\rho}\left(\Delta u_{2 R}\right)^{2} \leq \int_{B_{2 R} \times\{0\}} e^{\rho}\left(\Delta u_{2 R}\right)^{2} \\
& \quad+O\left(\int_{B_{2 R} \times[0, t]} e^{\rho}|\nabla \rho|^{2}\left|f_{2 R}\right|^{2}\left|\nabla u_{2 R}\right|^{2}+\int_{B_{2 R} \times[0, t]} e^{\rho}\left|\nabla f_{2 R}\right|^{2}\left|\nabla u_{2 R}\right|^{2}\right. \\
& \quad+\int_{B_{2 R} \times[0, t]} e^{\rho}\left|f_{2 R}\right|\left|\nabla u_{2 R}\right|^{2}\left|\triangle f_{2 R}\right|+\int_{B_{2 R} \times[0, t]} e^{\rho}\left|f_{2 R}\right|^{4}\left|\nabla u_{2 R}\right|^{2} \\
& \left.\quad+\int_{B_{2 R} \times[0, t]} e^{\rho}\left|\nabla\left(V_{2 R} u_{2 R}\right)\right|^{2}+\int_{B_{2 R} \times[0, t]} e^{\rho}\left|\nabla u_{2 R}\right|^{2}\left(\sum_{i=1}^{n}\left(f_{2 R}\right)_{i, i}\right)^{2}\right)
\end{aligned}
$$


Proof. It is easy to show that $\left|f_{2 R}\right|,\left|\nabla f_{2 R}\right|,\left|\nabla V_{2 R}\right|$ have linear growth while $\left|V_{2 R}\right|$ has quadratic growth, so we can apply Lemma 1.2 and Theorem 1.1 to get our estimates (i), (ii) and (iii) above.

THEOREM 1.3. Let $u_{2 R}$ be the solution of (1.53) where $R \geq 1$ woth inital condition $u_{2 R}(x, 0)=\psi_{2 R}(x)$. Assume that (1.44) - (1.47) hold i.e., $|f(x, t)| \leq c(1+|x|)$, $|\nabla f(x, t)| \leq c(1+|x|),|V(x, t)| \leq c\left(1+|x|^{2}\right)$ and $|\nabla V(x, t)| \leq c(1+|x|)$, where $c \geq 4$ is a constant. Assume further that $|\Delta f(x, t)| \leq c(1+|x|)$. Let $\delta$ and $\tilde{c}$ be positive constant such that $\widetilde{\widetilde{c}}:=\widetilde{c}+\delta<\min \left(\frac{1}{4}, \frac{\varepsilon_{1}^{2}}{4 \varepsilon_{1}^{3}+2}\right)$. Choose $T$ and $\varepsilon$ suitably small so that $T+\varepsilon<\delta$ and the conclusions (i), (ii) and (iii) in Lemma 1.3 hold for both

$$
\rho(x, t)=\frac{\widetilde{c}\left(1+|x|^{2}\right)}{t+\varepsilon} \quad \text { and } \quad \widetilde{\rho}(x, t)=\frac{\widetilde{\widetilde{c}}\left(1+|x|^{2}\right)}{t+\varepsilon}
$$

for any $0 \leq t \leq T$. Then for any $0 \leq t \leq T$
(i) $\int_{B_{2 R} \times\{t\}} e^{\rho} u_{2 R}^{2}$
(ii) $\int_{B_{2 R} \times\{t\}} e^{\rho}\left|\nabla u_{2 R}\right|^{2}$
(iii) $\int_{B_{2 R} \times\{t\}} e^{\rho}\left|\triangle u_{2 R}\right|^{2}$

are bounded above independent of $R$ and $t$.

Proof. By Lemma 1.3, we have

$$
\begin{aligned}
& \text { (i) } \int_{B_{2 R} \times\{t\}} e^{\rho} u_{2 R}^{2} \leq \int_{B_{2 R} \times\{0\}} e^{\rho} u_{2 R}^{2}=\int_{B_{2 R}} e^{\rho(x, 0)}\left(\psi_{2 R}(x)\right)^{2} \\
& \leq \int_{\mathbb{R}^{n}} e^{\rho(x, 0)}(\psi(x))^{2} \\
& \text { (ii) } \int_{B_{2 R} \times\{t\}} e^{\rho}\left|\nabla u_{2 R}\right|^{2} \leq \int_{B_{2 R} \times\{0\}} e^{\rho}\left|\nabla u_{2 R}\right|^{2}+\int_{0}^{t} \int_{B_{2 R}} e^{\rho(x, s)}\left(u_{2 R}(x, s)\right)^{2} \\
& \leq \int_{B_{2 R}} e^{\rho(x, 0)}\left|\nabla \psi_{2 R}(x)\right|^{2}+t \int_{B_{2 R}} e^{\rho(x, 0)}(\psi(x))^{2} \\
& \leq \int_{B_{2 R}} e^{\rho(x, 0)}(|\nabla \sigma||\psi|+|\nabla \psi|)^{2}+T \int_{B_{2 R}} e^{\rho(x, 0)}(\psi(x))^{2} \\
& \leq 2 \int_{B_{2 R}} e^{\rho(x, 0)}|\nabla \sigma|^{2}|\psi|^{2}+2 \int_{B_{2 R}} e^{\rho(x, 0)}|\nabla \psi|^{2}+T \int_{B_{2 R}} e^{\rho(x, 0)}(\psi(x))^{2} \\
& \leq 8 \int_{B_{2 R}} e^{\rho(x, 0)}|\psi|^{2}+2 \int_{B_{2 R}} e^{\rho(x, 0)}|\nabla \psi|^{2}+T \int_{B_{2 R}} e^{\rho(x, 0)}(\psi(x))^{2} \\
& =(8+T) \int_{\mathbb{R}^{n}} e^{\rho(x, 0)}|\psi|^{2}+2 \int_{\mathbb{R}^{n}} e^{\rho(x, 0)}|\nabla \psi|^{2} \\
& \text { (iii) } \int_{B_{2 R} \times\{t\}} e^{\rho}\left(\Delta u_{2 R}\right)^{2} \leq \int_{B_{2 R} \times\{0\}} e^{\rho}\left(\Delta u_{2 R}\right)^{2} \\
& +O\left(\int_{B_{2 R} \times[0, t]} e^{\rho}|\nabla \rho|^{2}\left|f_{2 R}\right|^{2}\left|\nabla u_{2 R}\right|^{2}+\int_{B_{2 R} \times[0, t]} e^{\rho}\left|\nabla f_{2 R}\right|^{2}\left|\nabla u_{2 R}\right|^{2}\right. \\
& +\int_{B_{2 R} \times[0, t]} e^{\rho}\left|f_{2 R}\right|\left|\nabla u_{2 R}\right|^{2}\left|\triangle f_{2 R}\right|+\int_{B_{2 R} \times[0, t]} e^{\rho}\left|f_{2 R}\right|^{4}\left|\nabla u_{2 R}\right|^{2} \\
& \left.+\int_{B_{2 R} \times[0, t]} e^{\rho}\left|\nabla\left(V_{2 R} u_{2 R}\right)\right|^{2}+\int_{B_{2 R} \times[0, t]} e^{\rho}\left|\nabla u_{2 R}\right|^{2}\left(\sum_{i=1}^{n}\left(f_{2 R}\right)_{i, i}\right)^{2}\right)
\end{aligned}
$$


We need to get upper estimate of the right hand side of the above inequality.

$$
\begin{aligned}
& \int_{B_{2 R} \times\{0\}} e^{\rho}\left(\triangle u_{2 R}\right)^{2}=\int_{B_{2 R}} e^{\rho(x, 0)}|\triangle(\sigma \psi)|^{2} \\
& =\int_{B_{2 R}} e^{\rho(x, 0)}((\triangle \psi) \sigma+2 \nabla \zeta \cdot \nabla \sigma+\psi \triangle \sigma)^{2} \\
& =\int_{B_{2 R}} e^{\rho(x, 0)}\left[(\triangle \psi)^{2} \sigma^{2}+4(\nabla \psi \cdot \nabla \sigma)^{2}+\psi^{2}(\triangle \sigma)^{2}+4(\triangle \psi) \sigma(\nabla \psi \cdot \nabla \sigma)\right. \\
& +2(\Delta \psi) \sigma \psi \Delta \sigma+4(\nabla \dot{\psi} \cdot \nabla \sigma) \psi \Delta \sigma] \\
& \leq \int_{B_{2 R}} e^{\rho(x, 0)}\left[|\Delta \psi|^{2} \sigma^{2}+4(\nabla \psi \cdot \nabla \sigma)^{2}+\psi^{2}(\Delta \sigma)^{2}\right] \\
& +\int_{B_{2 R}} e^{\rho(x, 0)}\left\{\left[|\Delta \psi|^{2} \sigma^{2}+4(\nabla \psi \cdot \nabla \sigma)^{2}\right]+\left[|\Delta \psi|^{2} \sigma^{2}+\psi^{2}(\Delta \sigma)^{2}\right]\right. \\
& \left.+\left[4(\nabla \psi \cdot \nabla \sigma)^{2}+\left(\psi^{2} \Delta \sigma\right)^{2}\right]\right\} \\
& =3 \int_{B_{2 R}} e^{\rho(x, 0)}\left[|\triangle \psi|^{2} \sigma^{2}+4(\nabla \psi \cdot \nabla \sigma)^{2}+\psi^{2}(\triangle \sigma)^{2}\right] \\
& \leq 3 \int_{B_{2 R}} e^{\rho(x, 0)}\left[\left(\left.\Delta \psi\right|^{2}+4|\nabla \psi|^{2}|\nabla \sigma|^{2}+\psi^{2}(\Delta \sigma)^{2}\right]\right. \\
& \leq 3 \int_{B_{2 R}} e^{\rho(x, 0)}|\Delta \psi|^{2}+48 \int_{B_{2 R}} e^{\rho(x .0)}|\nabla \psi|^{2}+12 \int_{B_{2 R}} e^{\rho(x, 0)} \psi^{2} \\
& \leq 3 \int_{\mathbb{R}^{n}} e^{\rho(x, 0)}|\Delta \psi|^{2}+48 \int_{\mathbb{R}^{n}} e^{\rho(x, 0)}|\nabla \psi|^{2}+12 \int_{\mathbb{R}^{n}} e^{\rho(x, 0)} \psi^{2} \\
& \left.\int_{B_{2 R} \times[0, t]} e^{\rho}|\nabla \rho|^{2} f_{2 R}\right|^{2}\left|\nabla u_{2 R}\right|^{2}=\int_{0}^{t} \int_{B_{2 R} \times\{s\}} e^{\rho} \frac{4 \widetilde{c}|x|^{2}}{(s+\varepsilon)^{2}}|\sigma f|^{2}\left|\nabla u_{2 R}\right|^{2} \\
& \left.\leq \int_{0}^{t} \frac{8 \widetilde{c}^{2} c^{2}}{(s+\varepsilon)^{2}} \int_{B_{2 R} \times\{s\}} e^{\rho}|x|^{2}(1+|x|)^{2}\left|\nabla u_{2 R}\right|^{2} \mid\right)
\end{aligned}
$$

Since $s+\varepsilon<\delta$, we have

$$
\begin{aligned}
|x|^{2}(1+|x|)^{2} & \leq 2|x|^{2}+2|x|^{4} \\
& \leq e^{\frac{\delta}{s+\varepsilon}}\left(1+\frac{\delta|x|^{2}}{s+\varepsilon}+\frac{\delta^{2}|x|^{4}}{(s+\varepsilon)^{2}}+\cdots\right) \\
& =e^{\frac{\delta}{s+\varepsilon}} e^{\frac{\delta|x|^{2}}{s+\varepsilon}}=e^{\frac{\delta\left(1+|x|^{2}\right)}{s+\varepsilon}}
\end{aligned}
$$

Hence

$$
\begin{aligned}
\int_{B_{2 R} \times[0, t]} & e^{\rho}|\nabla \rho|^{2}\left|f_{2 R}\right|^{2}\left|\nabla u_{2 R}\right|^{2} \\
& \leq \int_{0}^{t} \frac{8 \widetilde{c}^{2} c^{2}}{(s+\varepsilon)^{2}} \int_{B_{2 R} \times\{s\}} e^{\frac{\tilde{c}\left(1+|z|^{2}\right)}{s+s}} e^{\frac{\delta\left(1+|x|^{2}\right)}{s+\varepsilon}}\left(\left|\nabla u_{2 R}\right|^{2}\right) \\
& =\int_{0}^{t} \frac{8 \widetilde{c}^{2} c^{2}}{(s+\varepsilon)} \int_{B_{2 R} \times\{s\}} e^{\tilde{\rho}(x, s)}\left|\nabla u_{2 R}\right|^{2} \\
& \leq \int_{0}^{t} \frac{8 \widetilde{c}^{2} c^{2}}{(s+\varepsilon)} \int_{B_{2 R}} e^{\widetilde{\rho}(x, 0)}\left|\nabla \psi_{2 R}\right|^{2}
\end{aligned}
$$




$$
\begin{aligned}
& =8 \widetilde{c}^{2} c^{2} \frac{t}{\varepsilon(T+\varepsilon)} \int_{B_{2 R}} e^{\tilde{\rho}(x, 0)}|(\nabla \sigma) \psi+\sigma(\nabla \psi)|^{2} \\
& \leq \frac{16 \widetilde{c}^{2} c^{2} t}{\varepsilon(t+\varepsilon)} \int_{B_{2 R}} e^{\tilde{\rho}(x, 0)}\left(|\nabla \sigma|^{2} \psi^{2}+\sigma^{2}|\nabla \psi|^{2}\right) \\
& \leq \frac{16 \widetilde{c}^{2} c^{2} T}{\varepsilon(T+\varepsilon)} \int_{\mathbb{R}^{n}} e^{\tilde{\rho}(x, 0)}\left(4 \psi^{2}+|\nabla \psi|^{2}\right) \\
\int_{B_{2 R} \times[0, t]} & e^{\rho}\left|f_{2 R}\right|^{2}\left|\nabla u_{2 R}\right|^{2} \leq \int_{0}^{t} \int_{B_{2 R} \times\{s\}} e^{\rho}\left(\sum_{i=1}^{n}\left|(\nabla \sigma) f_{i}+\sigma \nabla f_{i}\right|^{2}\left|\nabla u_{2 R}\right|^{2}\right. \\
& \leq \int_{0}^{t} \int_{B_{2 R} \times\{s\}} e^{\rho} \sum_{i=1}^{n}\left(|\nabla \sigma|^{2} f_{i}^{2}+\sigma^{2}\left|\nabla f_{i}\right|^{2}\right)\left|\nabla u_{2 R}\right|^{2} \\
& \leq \int_{0}^{t} \int_{\left(B_{2 R}-B_{R}\right) \times\{s\}}\left(8 e^{\rho}|f|^{2}+2 e^{\rho}|\nabla f|^{2}\right)\left|\nabla u_{2 R}\right|^{2} \\
& \leq \int_{0}^{t} \int_{\left(B_{2 R}-B_{R}\right) \times\{s\}}\left(10 c^{2} e^{\rho}(1+|x|)^{2}\left|\nabla u_{2 R}\right|^{2}\right. \\
& \leq \int_{0}^{t} \int_{\left(B_{2 R}-B_{R}\right) \times\{s\}} 10 c^{2} e^{\rho} e^{\frac{\delta\left(1+|x|^{2}\right)}{s+\varepsilon}}\left|\nabla u_{2 R}\right|^{2} \\
& =\int_{0}^{t} \int_{\left(B_{2 R}-B_{R}\right) \times\{s\}} 10 c^{2} e^{\tilde{\rho}}\left|\nabla u_{2 R}\right|^{2} \\
& \leq 10 c^{2} \int_{0}^{t} \int_{B_{2 R}} e^{\tilde{\rho}(x, 0)}\left|\nabla \psi_{2 R}\right|^{2} \\
& \leq 10 c^{2} T \int_{\mathbb{R}^{n}} e^{\tilde{\rho}(x, 0)}\left(4 \psi^{2}+|\nabla \psi|^{2}\right)
\end{aligned}
$$

$$
\begin{aligned}
& \int_{B_{2 R} \times[0, t]} e^{\rho}\left|f_{2 R}\right|^{2}\left|\triangle f_{2 R}\right|\left|\nabla u_{2 R}\right|^{2}=\int_{0}^{t} \int_{B_{2 R} \times\{s\}} e^{\rho}|f \sigma| \sqrt{\sum_{i=1}^{n}\left|\triangle\left(\sigma f_{i}\right)\right|^{2}\left|\nabla u_{2 R}\right|^{2}} \\
& \leq \int_{0}^{t} \int_{\left(B_{2 R}-B_{R}\right) \times\{s\}} e^{\rho}|f| \sqrt{\sum_{i=1}^{n}\left[\left(\triangle f_{i}\right) \sigma+2\left(\nabla f_{i}\right) \cdot(\nabla \sigma)+f_{i} \triangle \sigma\right]^{2}\left|\nabla u_{2 R}\right|^{2}} \\
& \leq \int_{0}^{t} \int_{\left(B_{2 R}-B_{R}\right) \times\{s\}} \sqrt{3} e^{\rho}|f| \sqrt{\sum_{i=1}^{n}\left[\left|\triangle f_{i}\right|^{2} \sigma^{2}+4\left|\nabla f_{i}\right|^{2}|\nabla \sigma|^{2}+\left|f_{i}\right|^{2}|\triangle \sigma|^{2}\left|\nabla u_{2 R}\right|^{2}\right.} \\
& \leq \sqrt{3} \int_{0}^{t} \int_{\left(B_{2 R}-B_{R}\right) \times\{s\}} e^{\rho}|f|(|\sigma||\triangle f|+4|\nabla \sigma||\nabla f|+|\triangle \sigma||f|)\left|\nabla u_{2 R}\right|^{2} \\
& \leq \sqrt{3} \int_{0}^{t} \int_{\left(B_{2 R}-B_{R}\right) \times\{s\}} e^{\rho} c(1+|x|)(c+16 c+4 c)(1+|x|)\left|\nabla u_{2 R}\right|^{2} \\
& =\sqrt{3} \int_{0}^{t} \int_{\left(B_{2 R}-B_{R}\right) \times\{s\}} 21 c^{2} e^{\rho}(1+|x|)^{2}\left|\nabla u_{2 R}\right|^{2} \\
& \leq 21 \sqrt{3} c^{2} \int_{0}^{t} \int_{\left(B_{2 R}-B_{R}\right) \times\{s\}} e^{\rho} e^{\frac{\delta\left(1+|x|^{2}\right)}{s+\varepsilon}}\left|\nabla u_{2 R}\right|^{2}
\end{aligned}
$$




$$
\begin{aligned}
& \leq 21 \sqrt{3} c^{2} \int_{0}^{t} \int_{B_{2 R} \times\{s\}} e^{\tilde{\rho}}\left|\nabla u_{2 R}\right|^{2} \\
& \leq 21 \sqrt{3} c^{2} \int_{0}^{t} \int_{B_{2 R}} e^{\tilde{\rho}(x, 0)}\left|\nabla \psi_{2 R}\right|^{2} \\
& \leq 21 \sqrt{3} c^{2} T \int_{\mathbb{R}^{n}} e^{\tilde{\rho}(x, 0)}\left(4 \psi^{2}+|\nabla \psi|^{2}\right) \\
& \int_{B_{2 R} \times[0, t]} e^{\rho}\left|f_{2 R}\right|^{4}\left|\nabla u_{2 R}\right|^{2}=\int_{0}^{t} \int_{B_{2 R} \times\{s\}} e^{\rho} c^{4}(1+|x|)^{4}\left|\nabla u_{2 R}\right|^{2} \\
& \leq \int_{0}^{t} \int_{B_{2 R} \times\{s\}} e^{\rho} 8 c^{4}\left(1+|x|^{4}\right)\left|\nabla u_{2 R}\right|^{2} \\
& \leq \int_{0}^{t} \int_{B_{2 R} \times\{s\}} 8 c^{4} e^{\rho} e^{\frac{\delta\left(1+|x|^{2}\right)}{s+\varepsilon}}\left|\nabla u_{2 R}\right|^{2} \\
& =8 c^{4} \int_{0}^{t} \int_{B_{2 R} \times\{s\}} e^{\widetilde{\rho}}\left|\nabla u_{2 R}\right|^{2} \\
& \leq 8 c^{4} \int_{0}^{t} \int_{B_{2 R}} e^{\tilde{\rho}(x, 0)}\left|\nabla \psi_{2 R}\right|^{2} \\
& \leq 8 c^{4} T \int_{\mathbb{R}^{n}}^{\tilde{\rho}(x, 0)}\left(4 \psi^{2}+|\nabla \psi|^{2}\right)
\end{aligned}
$$

$$
\begin{aligned}
& \int_{B_{2 R} \times[0, t]} e^{\rho}\left|\nabla\left(V_{2 R} u_{2 R}\right)\right|^{2}=\int_{0}^{t} \int_{B_{2 R} \times\{s\}} e^{\rho}\left|\nabla\left(\sigma V u_{2 R}\right)\right| \\
& =\int_{0}^{t} \int_{B_{2 R} \times\{s\}} e^{\rho}\left|(\nabla \sigma) V u_{2 R}+\sigma(\nabla V) u_{2 R}+\sigma V\left(\nabla u_{2 R}\right)\right|^{2} \\
& \leq 3 \int_{0}^{t} \int_{B_{2 R} \times\{s\}} e^{\rho}\left(|\nabla \sigma|^{2}|V|^{2}\left|u_{2 R}\right|^{2}+|\sigma|^{2}|\nabla V|^{2}\left|u_{2 R}\right|^{2}+|\sigma|^{2}|V|^{2}\left|\nabla u_{2 R}\right|^{2}\right) \\
& \leq 3 \int_{0}^{t} \int_{\left(B_{2 R}-B_{R}\right) \times\{s\}} e^{\rho}\left(4 c^{2}\left(1+|x|^{2}\right)^{2}\left|u_{2 R}\right|^{2}+c^{2}(1+|x|)^{2}\left|u_{2 R}\right|^{2}\right. \\
& \left.\leq 3 \int_{0}^{t} \int_{\left(B_{2 R}-B_{R}\right) \times\{s\}} e^{\rho}\left(1+|x|^{2}\right)^{2}\left|\nabla u_{2 R}\right|^{2}\right) \\
& \leq 3 \int_{0}^{t} \int_{\left(B_{2 R}-B_{R}\right) \times\{s\}} e^{\rho}\left(\left.10 c^{2}\left(1+|x|^{4}\right)|| u_{2 R}\right|^{2}+2 c^{2}\left(1+|x|^{4}\right)\left|\nabla u_{2 R}\right|^{2}\right) \\
& \left.\leq 3 \int_{0}^{t} \int_{B_{2 R} \times\{s\}} e^{\rho} e^{\frac{\delta\left(1+|x|^{2}\right)}{s+\varepsilon}}\left(10 c^{2}\left|u_{2 R}\right|^{2}+2 c^{2}\left|\nabla u_{2 R}\right|^{2}\right)\left|u_{2 R}\right|^{2}+2 c^{2}\left(1+|x|^{4}\right)\left|\nabla u_{2 R}\right|^{2}\right) \\
& \leq 3 \int_{0}^{t} \int_{B_{2 R} \times\{s\}} e^{\tilde{\rho}\left(10 c^{2}\left|u_{2 R}\right|^{2}+2 c^{2}\left|\nabla u_{2 R}\right|^{2}\right)} \\
& \leq 3 T \int_{B_{2 R}} e^{\tilde{\rho}(x, 0)}\left(10 c^{2}\left|\psi_{2 R}\right|^{2}+2 c^{2}\left|\nabla \psi_{2 R}\right|^{2}\right) \\
& \leq 3 T \int_{\mathbb{R}^{n}} e^{\tilde{\rho}(x, 0)}\left(18 c^{2}|\psi|^{2}+2 c^{2}|\nabla \psi|^{2}\right)
\end{aligned}
$$




$$
\begin{aligned}
& \int_{B_{2 R} \times[0, t]} e^{\rho}\left|\nabla u_{2 R}\right|^{2}\left(\sum_{i=1}^{n}\left(f_{2 R}\right)_{i, i}\right)^{2} \leq \int_{0}^{t} \int_{B_{2 R} \times\{s\}} n e^{\rho}\left|\nabla u_{2 R}\right|^{2} \sum_{i=1}^{n}\left(f_{2 R}\right)_{i, i}^{2} \\
& \leq \int_{0}^{t} \int_{B_{2 R} \times\{s\}} n e^{\rho}|\nabla f|^{2}\left|\nabla u_{2 R}\right|^{2} \\
& \leq \int_{0}^{t} \int_{B_{2 R} \times\{s\}} n e^{\rho} c^{2}(1+|x|)^{2}\left|\nabla u_{2 R}\right|^{2} \\
& \leq \int_{0}^{t} \int_{B_{2 R} \times\{s\}} 2 n c^{2} e^{\rho}\left(1+|x|^{2}\right)\left|\nabla u_{2 R}\right|^{2} \\
& \leq \int_{0}^{t} \int_{B_{2 R} \times\{s\}} 2 n c^{2} e^{\tilde{\rho}}\left|\nabla u_{2 R}\right|^{2} \\
& \leq \int_{0}^{t} \int_{B_{2 R}} 2 n c^{2} e^{\tilde{\rho}(x, 0)}\left|\nabla \psi_{2 R}\right|^{2} \\
& \leq 2 n c^{2} T \int_{\mathbb{R}^{n}} e^{\tilde{\rho}(x, 0)}\left(4 \psi^{2}+|\nabla \psi|^{2}\right)
\end{aligned}
$$

We see from Theorem 1.3 that for $0 \leq t \leq T$, we have estimates of $\int_{B_{2 R} \times\{t\}} e^{\rho} u_{2 R}^{2}$, $\int_{B_{2 R} \times\{t\}} e^{\rho}\left|\nabla u_{2 R}\right|^{2}$ and $\int_{B_{2 R} \times\{t\}} e^{\rho}\left|\triangle u_{2 R}\right|^{2}$ which are independent of $R$. Hence we can take $R \rightarrow \infty$ and obtain a global solution $u$ up to time $T$.

THEOREM 1.4. Assume that $|f(x, t)| \leq c(1+|x|),|\nabla f(x, t)| \leq c(1+|x|),|\triangle f(x, t)|$ $\leq c(1+|x|),|V(x, t)| \leq c\left(1+|x|^{2}\right)$ and $|\nabla V(x, t)| \leq c(1+|x|)$ where $c \geq 4$ is a constant. Let $\delta$ and $\tilde{c}$ be positive constant such that $\widetilde{\widetilde{c}}:=\tilde{c}+\delta<\min \left(\frac{1}{4}, \frac{\varepsilon_{1}^{2}}{4 \varepsilon_{1}^{2}+2}\right)$. Choose $T$ and $\varepsilon$ suitably small so that $T+\varepsilon<\delta$ and the conclusions (i), (ii) and (iii) of Lemma 1.3 hold for both

$$
\rho(x, t)=\frac{\widetilde{c}\left(1+|x|^{2}\right)}{t+\varepsilon} \quad \text { and } \quad \widetilde{\rho}(x, t)=\frac{\widetilde{\widetilde{c}}\left(1+|x|^{2}\right)}{t+\varepsilon}
$$

for any $0 \leq t \leq T$. Then for any initial data $\psi(x)$ with $\int_{\mathbb{R}^{n}} e^{\rho}\left(|\psi|^{2}+|\nabla \psi|^{2}+|\triangle \psi|^{2}\right)<$ $\infty$, there exist a solution of the equation $\frac{\partial u}{\partial t}=\Delta u+\sum_{i=1}^{n} f_{i} \frac{\partial u}{\partial x_{i}}-V u$ up to time $T$ and $u(x, 0)=\psi(x)$.

2. Gradient estimate of the solution. We shall first derive the following maximal principle for the parabolic equation which we are interested in.

THEOREM 2.1. Let $u>0$ be a positive solution of the equation.

$$
\frac{\partial u}{\partial t}(x, t)=\Delta u(x, t)+\sum_{i=1}^{n} f_{i}(x, t) \frac{\partial u}{\partial x_{i}}(x, t)-V(x, t) u(x, t)
$$


defined on a compact domain $\Omega$. Let $\varphi(x, t)=-\log u_{\Omega}(x, t)$ and

$$
\Psi=\varphi_{t}+|\nabla \varphi|^{2}-\sum_{j=1}^{n} f_{j} \frac{\partial \varphi}{\partial x_{j}}-V-\alpha(t) \sqrt{|\nabla \varphi|^{2}+\beta(x, t)}-\frac{n}{2 t}-2 \sqrt{n} c(t)
$$

where

$$
\begin{aligned}
& 0<\alpha(t) \leq \frac{1}{2} \sqrt{\beta(x, t)}, \\
& |\nabla f(x, t)| \leq c(t)
\end{aligned}
$$

Assume that there exists $c_{1}(t)$ such that

$$
\begin{aligned}
& |f(x, t)|^{2} \leq c(t)^{2}(1+|x|)^{2} \leq c_{1}(t) \beta(x, t) \\
& |\nabla \beta(x, t)| \leq c_{1}(t) \sqrt{\beta(x, t)}
\end{aligned}
$$

Suppose $\psi<0$ on $\Omega \times\left[0, t_{0}\right)$ and the following inequalities hold

$$
\begin{aligned}
& \frac{3 \beta}{2 t}+\frac{\beta_{t}}{2}-\frac{n}{4 t^{2}}>0 \\
& \alpha^{\prime}(t)+\frac{3 \alpha c}{2 \sqrt{n}}-\alpha \lambda-\sqrt{n}|\Delta f|-\frac{\alpha|\nabla V|}{2 \sqrt{\beta}} \\
& -\frac{1}{2}\left(c_{1}+c_{1}^{3 / 2}\right) \alpha \geq 0 \\
& 2 \sqrt{n} c^{\prime}(t)+\frac{3 c^{2}}{8}-\Delta V-\frac{1}{6} \sum_{i, j=1}^{n}\left(\frac{f_{i, j}+f_{j, i}}{2}\right)^{2}+\frac{3 \sqrt{n} c}{4 t}-\frac{\alpha \Delta \beta}{\sqrt{\beta}} \geq 0
\end{aligned}
$$

where $\lambda$ is the absolute value of the greatest eigenvalue of $\left(\frac{f_{i, j}+f_{i, i}}{2}\right)$. Then $\Psi$ cannot have an interior maximum with $\Psi=0$ at $t=t_{0}$.

Proof.

$$
\begin{aligned}
\frac{\partial \varphi}{\partial t} & =-\frac{1}{u} \log u, \quad \frac{\partial \varphi}{\partial x_{i}}=-\frac{1}{u} \frac{\partial u}{\partial x_{i}} \\
\frac{\partial^{2} \varphi}{\partial x_{i}^{2}} & =\frac{1}{u^{2}}\left(\frac{\partial u}{\partial x_{i}}\right)^{2}-\frac{1}{u} \frac{\partial^{2} u}{\partial x_{i}^{2}}
\end{aligned}
$$

Hence

$$
\begin{gathered}
\Delta \varphi-|\nabla \varphi|^{2}=\frac{1}{u^{2}}|\nabla u|^{2}-\frac{1}{u} \Delta u-\frac{1}{u^{2}}|\nabla u|^{2}=-\frac{1}{u} \Delta u \\
\varphi_{t}=-\frac{1}{u} u_{t}=-\frac{1}{u}\left(\Delta u+\sum_{i=1}^{n} f_{i} u_{i}-V u\right) \\
=\Delta \varphi-|\nabla \varphi|^{2}+\sum_{i=1}^{n} f_{i} \varphi_{i}+V \\
\Psi_{t}=\varphi_{t t}+2 \sum_{j=1}^{n}\left(\varphi_{j}\right)_{t} \varphi_{j}-\sum_{j=1}^{n}\left(f_{i}\right)_{t} \varphi_{j}-\sum_{j=1}^{n} f_{j}\left(\varphi_{j}\right)_{t}-V_{t}-\alpha_{t} \sqrt{|\nabla \varphi|^{2}+\beta}
\end{gathered}
$$




$$
\begin{aligned}
& -\frac{\alpha}{2}\left[\sum_{j=1}^{n} 2 \varphi_{j}\left(\varphi_{j}\right)_{t}+\beta_{t}\right]\left(|\nabla \varphi|^{2}+\beta\right)^{-\frac{1}{2}}+\frac{n}{2 t^{2}}-2 \sqrt{n} c_{t} \\
\Psi_{i}= & \left(\varphi_{i}\right)_{t}+2 \sum_{j=1}^{n} \varphi_{j i} \varphi_{j}-\sum_{j=1}^{n} f_{j, i} \varphi_{j}-\sum_{j=1}^{n} f_{j} \varphi_{j i}-V_{i} \\
& -\frac{\alpha}{2}\left[2 \sum_{j=1}^{n} \varphi_{j} \varphi_{j i}+\beta_{i}\right]\left(|\nabla \varphi|^{2}+\beta\right)^{-\frac{1}{2}} \\
\Psi_{i i}= & \left(\varphi_{i i}\right)_{t}+2 \sum_{j=1}^{n} \varphi_{j i}^{2}+2 \sum_{j=1}^{n} \varphi_{j i i} \varphi_{j}-\sum_{j=1}^{n} f_{j, i i} \varphi_{j}-2 \sum_{j=1}^{n} f_{j, i} \varphi_{j i} \\
& -\sum_{j=1}^{n} f_{j} \varphi_{j i i}-V_{i i}-\frac{\alpha}{2}\left[2 \sum_{j=1}^{n} \varphi_{j i}^{2}+2 \sum_{j=1}^{n} \varphi_{j} \varphi_{j i i}+\beta_{i i}\right]\left(|\nabla \varphi|^{2}+\beta\right)^{-\frac{1}{2}} \\
& +\frac{\alpha}{4}\left(2 \sum_{j=1}^{N} \varphi_{j} \varphi_{j i}+\beta_{i}\right)^{2}\left(|\nabla \varphi|^{2}+\beta\right)^{-3 / 2} \\
\triangle \Psi= & (\Delta \varphi)_{t}+2 \sum_{i, j=1}^{n} \varphi_{j i}^{2}+2 \sum_{j=1}^{n}(\triangle \varphi)_{j} \varphi_{j}-\sum_{j=1}^{n}\left(\Delta f_{j}\right) \varphi_{j}-2 \sum_{i, j=1}^{n} f_{j, i} \varphi_{j i} \\
& -\sum_{j=1}^{n} f_{j} \Delta \varphi_{j}-\Delta V-\left[\alpha \sum_{i, j=1}^{n} \varphi_{j i}^{2}+\alpha \sum_{j=1}^{n} \varphi_{j} \Delta \varphi_{j}+\frac{\alpha}{2} \Delta \beta\right]\left(|\nabla|^{2}+\beta\right)^{-\frac{1}{2}} \\
& +\alpha \sum_{i=1}^{n}\left(\sum_{j=1}^{n} \varphi_{j} \varphi_{j i}+\frac{\beta_{i}}{2}\right)^{2}\left(|\nabla \varphi|^{2}+\beta\right)^{-3 / 2}
\end{aligned}
$$

By computation, we have

$$
\begin{aligned}
= & -\left(|\nabla \varphi|^{2}-\sum_{i=1}^{n} f_{i} \varphi_{i}-V\right)_{t}-2 \sum_{i, j=1}^{n} \varphi_{j i}^{2}-2 \sum_{j=1}^{n} \varphi_{j}\left(|\nabla \varphi|^{2}-\sum_{i=1}^{n} f_{i} \varphi_{i}-V\right)_{j} \\
& +2 \sum_{i, j=1}^{n} f_{j, i} \varphi_{j i}+\sum_{j=1}^{n} f_{j}\left(|\nabla \varphi|^{2}-\sum_{i=1}^{n} f_{i} \varphi_{i}-V\right)_{j}+\sum_{j=1}^{n}\left[-\left(f_{j}\right)_{t}+\Delta f_{j}\right] \varphi_{j} \\
& +\Delta V-V_{t}+\frac{\alpha \sum_{j=1}^{n} \varphi_{j}\left(|\nabla \varphi|^{2}-\sum_{i=1}^{n} f_{i} \varphi_{i}-V\right)_{j}}{\sqrt{|\nabla \varphi|^{2}+\beta}}+\frac{\alpha \sum_{i, j=1}^{n} \varphi_{i j}^{2}}{\sqrt{|\nabla \varphi|^{2}+\beta}} \\
& -\frac{\frac{\alpha}{2}\left(\beta_{t}-\Delta \beta\right)}{\sqrt{|\nabla \varphi|^{2}+\beta}-\frac{\alpha \sum_{i=1}^{n}\left(\sum_{j=1}^{n} \varphi_{j} \varphi_{j i}+\frac{\beta_{i}}{2}\right)^{2}}{\left(|\nabla \varphi|^{2}+\beta\right)^{3 / 2}}-\alpha_{t} \sqrt{|\nabla \varphi|^{2}+\beta}+\frac{n}{2 t^{2}}-2 \sqrt{n} c_{t}} \\
= & -2 \sum_{j=1}^{n}\left(\varphi_{t}\right)_{j} \varphi_{j}+\sum_{i=1}^{n}\left(f_{i}\right)_{t} \varphi_{i}+\sum_{i=1}^{n} f_{i}\left(\varphi_{t}\right)_{i}+V_{t}-2 \sum_{i, j=1}^{n} \varphi_{j i}^{2} \\
& -2 \sum_{j=1}^{n} \varphi_{j}\left(\Psi-\varphi_{t}+\alpha \sqrt{|\nabla \varphi|^{2}+\beta}+\frac{n}{2 t}+c\right)_{j}+2 \sum_{i, j=1}^{n} f_{j i} \varphi_{j i} \\
& +\sum_{j=1}^{n} f_{j}\left(\Psi-\varphi_{t}+\alpha \sqrt{|\nabla \varphi|^{2}+\beta}+\frac{n}{2 t}+c\right)_{j}+\sum_{j=1}^{n}\left[-\left(f_{j}\right)_{t}+\Delta f_{j}\right] \varphi_{j}
\end{aligned}
$$




$$
\begin{aligned}
& +\Delta V-V_{t}+\frac{\alpha \sum_{j=1}^{n} \varphi_{j}\left(|\nabla \varphi|^{2}-\sum_{j=1}^{n} f_{i} \varphi_{i}-V\right)_{j}}{\sqrt{|\nabla \varphi|^{2}+\beta}}+\frac{\alpha \sum_{i, j=1}^{n} \varphi_{i j}^{2}}{\sqrt{\left|\nabla \varphi^{2}\right|+\beta}} \\
& -\frac{\frac{\alpha}{2}\left(\beta_{t}-\Delta \beta\right)}{\sqrt{|\nabla \varphi|^{2}+\beta}}-\frac{\alpha \sum_{i=1}^{n}\left(\sum_{j=1}^{n} \varphi_{j} \varphi_{j i}+\frac{\beta_{i}}{2}\right)^{2}}{\left(|\nabla \varphi|^{2}+\beta\right)^{3 / 2}}-\left(\alpha_{t}-\Delta \alpha\right) \sqrt{|\nabla \varphi|^{2}+\beta} \\
& +\frac{n}{2 t^{2}}-2 \sqrt{n} c_{t} \\
& \left.=-2 \sum_{i, j=1}^{n} \varphi_{j i}^{2}-2 \sum_{j=1}^{n} \varphi_{j} \Psi_{j}-\alpha \sum_{j=1}^{n} \varphi_{j}\left(\sum_{i=1}^{n} 2 \varphi_{i} \varphi_{i j}+\beta_{j}\right)\left(|\nabla \varphi|^{2}\right)+\beta\right)^{-\frac{1}{2}} \\
& +2 \sum_{i, j=1}^{n} f_{j, i} \varphi_{j i}+\sum_{j=1}^{n} f_{j} \Psi_{j} \\
& +\frac{\alpha}{2} \sum_{j=1}^{n} f_{j}\left(\sum_{i=1}^{n} 2 \varphi_{i} \varphi_{i j}+\beta_{j}\right)\left(|\nabla \varphi|^{2}+\beta\right)^{-\frac{1}{2}}+\sum_{j=1}^{n}\left(\Delta f_{j}\right) \varphi_{j}+\Delta V \\
& +\frac{\alpha \sum_{j=1}^{n} \varphi_{j}\left(\sum_{i=1}^{n} 2 \varphi_{i} \varphi_{i j}-\sum_{i=1}^{n} f_{i, j} \varphi_{i}-\sum_{i=1}^{n} f_{i} \varphi_{i j}-V_{j}\right)}{\sqrt{|\nabla \varphi|^{2}+\beta}}+\frac{\alpha \sum_{i, j=1}^{n} \varphi_{i j}^{2}}{\sqrt{|\nabla \varphi|^{2}+\beta}} \\
& -\frac{\frac{\alpha}{2}\left(\beta_{t}-\Delta \beta\right)}{\sqrt{|\nabla \varphi|^{2}+\beta}}-\frac{\alpha \sum_{i=1}^{n}\left(\sum_{j=1}^{n} \varphi_{j} \varphi_{j i}+\frac{\beta_{i}}{2}\right)^{2}}{\left(|\nabla \varphi|^{2}+\beta\right)^{3 / 2}}-\alpha_{t} \sqrt{|\nabla \varphi|^{2}+\beta}+\frac{n}{2 t^{2}}-2 \sqrt{n} C_{t} \\
& =-2 \sum_{j=1}^{n} \varphi_{j} \Psi_{j}+\sum_{j=1}^{n} f_{j} \Psi_{j}-2 \sum_{i, j=1}^{n} \varphi_{j i}^{2}+2 \sum_{i, j=1}^{n} f_{j, i} \varphi_{j i}+\sum_{j=1}^{n}\left(\triangle f_{j}\right) \varphi_{j} \\
& +\Delta V-\frac{\alpha \sum_{i, j=1}^{n} f_{i, j} \varphi_{i} \varphi_{j}+\alpha \sum_{j=1}^{n} \varphi_{j} V_{j}}{\sqrt{|\nabla \varphi|^{2}+\beta}}+\frac{\alpha \sum_{i, j=1}^{n} \varphi_{i j}^{2}}{\sqrt{|\nabla \varphi|^{2}+\beta}}-\frac{\frac{\alpha}{2}\left(\beta_{t}-\Delta \beta\right)}{\sqrt{|\nabla \varphi|^{2}+\beta}} \\
& -\frac{\alpha \sum_{i=1}^{n}\left(\sum_{j=1}^{n} \varphi_{i} \varphi_{j i}+\frac{\beta_{i}}{2}\right)^{2}}{\left(|\nabla \varphi|^{2}+\beta\right)^{3 / 2}}-\alpha_{t} \sqrt{|\nabla \varphi|^{2}+\beta}+\frac{n}{2 t^{2}}-2 \sqrt{n} c_{t} \\
& -\frac{\alpha \sum_{j=1}^{n} \varphi_{j} \beta_{j}}{\sqrt{|\nabla \varphi|^{2}+\beta}}+\frac{\frac{\alpha}{2} \sum_{j=1}^{n} f_{j} \beta_{j}}{\sqrt{|\nabla \varphi|^{2}+\beta}} \\
& =-2 \sum_{j=1}^{n} \varphi_{j} \Psi_{j}+\sum_{j=1}^{n} f_{j} \Psi_{j}-2 \sum_{i, j=1}^{n} \varphi_{j i}^{2}+2 \sum_{i, j=1}^{n} f_{j, i} \varphi_{j i}+\sum_{j=1}^{n}\left(\Delta f_{j}\right) \varphi_{j}+\Delta V \\
& -\frac{\alpha \sum_{i, j=1}^{n} f_{i, j} \varphi_{i} \varphi_{j}+\alpha \sum_{j=1}^{n} \varphi_{j} V_{j}}{\sqrt{|\nabla \varphi|^{2}+\beta}}+\frac{\alpha \sum_{i, j=1}^{n} \varphi_{i j}^{2}}{\sqrt{|\nabla \varphi|^{2}+\beta}}-\frac{\frac{\alpha}{2}\left(\beta_{t}-\Delta \beta\right)}{\sqrt{|\nabla \varphi|^{2}+\beta}} \\
& -\frac{\alpha \sum_{i=1}^{n}\left(\sum_{j=1}^{n} \varphi_{j} \varphi_{j i}+\frac{\beta_{i}}{2}\right)^{2}}{\left(|\nabla \varphi|^{2}+\beta\right)^{3 / 2}}-\alpha_{t} \sqrt{|\nabla \varphi|^{2}+\beta}+\frac{n}{2 t^{2}}-2 \sqrt{n} c_{t} \\
& -\frac{\alpha \sum_{j=1}^{n} \varphi_{j} \beta_{j}}{\sqrt{|\nabla \varphi|^{2}+\beta}}+\frac{\frac{\alpha}{2} \sum_{j=1}^{n} f_{j} \beta_{j}}{\sqrt{|\nabla \varphi|^{2}+\beta}}
\end{aligned}
$$

Recall that $\Psi<0$ for $0 \leq t<t_{0}$. If $\Psi=0$ at some interior point when $t=t_{0}$, then at such a point, we have

$$
\begin{aligned}
& \Psi_{t}\left(x_{0}, t_{0}\right) \geq 0, \quad \nabla \Psi\left(x_{0}, t_{0}\right)=0, \quad \Delta \Psi\left(x_{0}, t_{0}\right) \leq 0 \\
& \triangle \varphi\left(x_{0}, t_{0}\right)=\alpha\left(x_{0}, t_{0}\right) \sqrt{\left|\nabla \varphi\left(x_{0}, t_{0}\right)\right|^{2}+\beta\left(x_{0}, t_{0}\right)}+\frac{n}{2 t_{0}}+2 \sqrt{n} c\left(t_{0}\right)
\end{aligned}
$$

(2.13) follows from (2.2) and (2.10). 
In view of the arithmetic-geometric inequality $\sum_{i=1}^{n} a_{i}^{2} \geq \frac{1}{n}\left(\sum_{i=1}^{n} a_{i}\right)^{2}$, we have at the point $\left(x_{0}, t_{0}\right)$.

$$
\left(2-\frac{\alpha}{\sqrt{|\nabla \varphi|^{2}+\beta}}\right) \sum_{i, j=1}^{n}\left[\varphi_{i j}-\frac{1}{2}\left(2-\frac{\alpha}{\sqrt{|\nabla \varphi|^{2}+\beta}}\right)^{-1}\left(f_{i, j}+f_{j, i}\right)\right]^{2}
$$$$
\geq \frac{1}{n}\left(2-\frac{\alpha}{\sqrt{|\nabla \varphi|^{2}+\beta}}\right)\left[\Delta \varphi-\left(2-\frac{\alpha}{\sqrt{|\nabla \varphi|^{2}+\beta}}\right)^{-1} \sum_{i=1}^{n} f_{i, i}\right]^{2}
$$$$
=\frac{1}{n}\left(2-\frac{\alpha}{\sqrt{|\nabla \varphi|^{2}+\beta}}\right)\left[\alpha \sqrt{|\nabla \varphi|^{2}+\beta}+\frac{n}{2 t}+2 \sqrt{n} c-\left(2-\frac{\alpha}{\sqrt{|\nabla \varphi|^{2}+\beta}}\right)^{-1} \sum_{i=1}^{n} f_{i, i}\right]^{2}
$$$$
=\frac{1}{n}\left(2-\frac{\alpha}{\sqrt{|\nabla \varphi|^{2}+\beta}}\right)\left[\alpha^{2}\left(|\nabla \varphi|^{2}+\beta\right)+\frac{n}{t} \alpha \sqrt{|\nabla \varphi|^{2}+\beta}+\frac{n^{2}}{4 t^{2}}\right.
$$

$$
\begin{gathered}
+\left(2 \sqrt{n} c-\left(2-\frac{\alpha}{\sqrt{|\nabla \varphi|^{2}+\beta}}\right)^{-1} \sum_{i=1}^{n} f_{i, i}\right)^{2}+\left(2 \alpha \sqrt{|\nabla \varphi|^{2}+\beta}+\frac{n}{t}\right) \\
\left.\left(2 \sqrt{n} c-\left(2-\frac{\alpha}{\sqrt{|\nabla \varphi|^{2}+\beta}}\right)^{-1} \sum_{i=1}^{n} f_{i, i}\right)\right]^{2}
\end{gathered}
$$

On the other hand from (2.11) and the fact that $\psi_{t}-\Delta \psi \geq 0$ at the point $\left(x_{0}, t_{0}\right)$, we have

$$
\begin{aligned}
& \left(2-\frac{\alpha}{\sqrt{|\nabla \varphi|^{2}+\beta}}\right) \sum_{i, j=1}^{n}\left[\varphi_{i j}-\frac{1}{2}\left(2-\frac{\alpha}{\sqrt{|\nabla \varphi|^{2}+\beta}}\right)^{-1}\left(f_{i, j}+f_{j, i}\right)\right]^{2} \\
\leq & \sum_{j=1}^{n}\left(\Delta f_{j}\right) \varphi_{j}+\Delta V+\frac{1}{4}\left(2-\frac{\alpha}{\sqrt{|\nabla \varphi|^{2}+\beta}}\right)^{-1} \sum_{i, j=1}^{n}\left(f_{i, j}+f_{j, i}\right)^{2} \\
- & \frac{\alpha \sum_{i, j=1}^{n} f_{i, j} \varphi_{i} \varphi_{j}+\alpha \sum_{j=1}^{n} \varphi_{j} V_{j}}{\sqrt{|\nabla \varphi|^{2}+\beta}}-\frac{\frac{\alpha}{2}\left(\beta_{t}-\Delta \beta\right)}{\sqrt{|\nabla \varphi|^{2}+\beta}} \\
- & \frac{\alpha \sum_{i=1}^{n}\left(\sum_{j=1}^{n} \varphi_{j} \varphi_{j i}+\frac{\beta_{i}}{2}\right)^{2}}{\left(|\nabla \varphi|^{2}+\beta\right)^{3 / 2}}-\alpha_{t} \sqrt{|\nabla \varphi|^{2}+\beta}+\frac{n}{2 t^{2}}-2 \sqrt{n} c_{t} \\
- & \frac{\alpha \sum_{j=1}^{n} \varphi_{j} \beta_{j}}{\sqrt{|\nabla \varphi|^{2}+\beta}}+\frac{\frac{\alpha}{2} \sum_{j=1}^{n} f_{j} \beta_{j}}{\sqrt{|\nabla \varphi|^{2}+\beta}} \\
\leq & \sum_{j=1}^{n}\left(\Delta f_{j}\right) \varphi_{j}+\Delta V+\frac{1}{4}\left(2-\frac{\alpha}{\sqrt{|\nabla \varphi|^{2}+\beta}}\right)^{-1} \sum_{i, j=1}^{n}\left(f_{i, j}+f_{j, i}\right)^{2} \\
- & \frac{\alpha \sum_{i, j=1}^{n} f_{i, j} \varphi_{i} \varphi_{j}+\alpha \sum_{j=1}^{n} \varphi_{j} V_{j}}{\sqrt{|\nabla \varphi|^{2}+\beta}}-\frac{\frac{\alpha}{2}\left(\beta_{t}-\Delta \beta\right)}{\sqrt{|\nabla \varphi|^{2}+\beta}}-\alpha_{t} \sqrt{|\nabla \varphi|^{2}+\beta} \\
+ & \frac{n}{2 t^{2}}-2 \sqrt{n} c_{t}-\frac{\alpha \sum_{j=1}^{n} \varphi_{j} \beta_{j}}{\sqrt{|\nabla \varphi|^{2}+\beta}}+\frac{\frac{\alpha}{2} \sum_{j=1}^{n} f_{j} \beta_{j}}{\sqrt{|\nabla \varphi|^{2}+\beta}}
\end{aligned}
$$

since $\alpha>0$ by assumption. Equation (2.14) and (2.15) imply

$$
\frac{1}{n}\left(2-\frac{\alpha}{\sqrt{|\nabla \varphi|^{2}+\beta}}\right)\left[\alpha^{2}\left(|\nabla \varphi|^{2}+\beta\right)+\frac{n \alpha}{t} \sqrt{|\nabla \varphi|^{2}+\beta}+\frac{n^{2}}{4 t^{2}}\right.
$$




$$
\begin{aligned}
& +\left(2 \sqrt{n} c-\left(2-\frac{\alpha}{\sqrt{|\nabla \varphi|^{2}+\beta}}\right)^{-1} \sum_{i=1}^{n} f_{i, i}\right)^{2}+\left(2 \alpha \sqrt{|\nabla \varphi|^{2}+\beta}+\frac{n}{t}\right)(2 \sqrt{n} c \\
& \left.\left.-\left(2-\frac{\alpha}{\sqrt{|\nabla \varphi|^{2}+\beta}}\right)^{-1} \sum_{i=1}^{n} f_{i, i}\right)\right] \\
& \leq \sum_{j=1}^{n}\left(\Delta f_{j}\right) \varphi_{j}+\Delta V+\frac{1}{4}\left(2-\frac{\alpha}{\sqrt{|\nabla \varphi|^{2}+\beta}}\right)^{-1} \sum_{i, j=1}^{n}\left(f_{i, j}+f_{j, i}\right)^{2} \\
& -\frac{\alpha \sum_{i, j=1}^{n} f_{i, j} \varphi_{i} \varphi_{j}+\alpha \sum_{j=1}^{n} \varphi_{j} V_{j}}{\sqrt{|\nabla \varphi|^{2}+\beta}-\frac{\alpha\left(\beta_{t}-\Delta \beta\right)}{2 \sqrt{|\nabla \varphi|^{2}+\beta}}-\alpha_{t} \sqrt{|\nabla \varphi|^{2}+\beta}} \\
& +\frac{n}{2 t^{2}}-2 \sqrt{n} c_{t}-\frac{\alpha \sum_{j=1}^{n} \varphi_{j} \beta_{j}}{\sqrt{|\nabla \varphi|^{2}+\beta}}+\frac{\alpha \sum_{j=1}^{n} f_{j} \beta_{j}}{2 \sqrt{|\nabla \varphi|^{2}+\beta}}
\end{aligned}
$$

Let $z=\sqrt{|\nabla \varphi|^{2}+\beta}$. In view of (2.3), we have

$$
2-\frac{\alpha}{z} \geq \frac{3}{2}
$$

Observe that

$$
|\nabla f|=\sqrt{\sum_{i=1}^{n}\left|\nabla f_{i}\right|^{2}} \geq \sqrt{\sum_{i=1}^{n}\left(\frac{\partial f_{i}}{\partial x_{i}}\right)^{2}} \geq \frac{1}{\sqrt{n}} \sum_{i=1}^{n} \frac{\partial f_{i}}{\partial x_{i}}
$$

Hence

$$
\begin{aligned}
2 \sqrt{n} c-\left(2-\frac{\alpha}{z}\right)^{-1} \sum_{i=1}^{n} f_{i, i} & \geq 2 \sqrt{n} c-\frac{3}{2} \sqrt{n}|\nabla f| \\
& \geq 2 \sqrt{n} c-\frac{3}{2} \sqrt{n} c=\frac{\sqrt{n}}{2} c
\end{aligned}
$$

The left hand side of (2.16) can be estimated from below as follow

$$
\begin{gathered}
\frac{1}{n}\left(2-\frac{\alpha}{z}\right)\left[\alpha z^{2}+\frac{n}{t} \alpha z+\frac{n^{2}}{4 t^{2}}+\left(2 \sqrt{n} c-\left(2-\frac{\alpha}{z}\right)^{-1} \sum_{i=1}^{n} f_{i, i}\right)^{2}\right. \\
\left.+\left(2 \alpha z+\frac{n}{t}\right)\left(2 \sqrt{n} c-\left(2-\frac{\alpha}{z}\right)^{-1} \sum_{i=1}^{n} f_{i, i}\right)\right] \\
\geq \frac{3}{2 n}\left[\alpha^{2} z^{2}+\frac{n \alpha z}{t}+\frac{n}{4} c^{2}+\left(2 \alpha z+\frac{n}{t}\right) \frac{\sqrt{n}}{2} c\right]+\frac{1}{n}\left(2-\frac{\alpha}{z}\right) \frac{n^{2}}{4 t^{2}} \\
=\frac{3 \alpha^{2} z^{2}}{2 n}+\frac{3 \alpha z}{2 t}+\frac{3}{8} c^{2}+\frac{3}{2 \sqrt{n}} \alpha z c+\frac{3 \sqrt{n}}{4} \frac{c}{t}+\frac{n}{2 t^{2}}-\frac{n \alpha}{4 t^{2} z}
\end{gathered}
$$

(2.6) implies

$$
2|\nabla \varphi||\triangle \beta| \leq 2 c_{1}|\nabla \varphi| \sqrt{\beta} \leq c_{1}\left(|\nabla \varphi|^{2}+\beta\right)
$$

which is equivalent to

$$
\frac{|\nabla \varphi||\nabla \beta|}{|\nabla \varphi|^{2}+\beta} \leq \frac{c_{1}}{2}
$$


In view of (2.5), (2.6) and the above inequality, the right hand side of (2.16) can be estimated from above as follow.

$$
\begin{aligned}
& \sum_{j=1}^{n}\left(\triangle f_{j}\right) \varphi_{j}+\Delta V+\frac{1}{4}\left(2-\frac{\alpha}{z}\right)^{-1} \sum_{i, j=1}^{n}\left(f_{i, j}+f_{j, i}\right)^{2} \\
& -\frac{1}{z}\left(\alpha \sum_{i, j=1}^{n} f_{i, j} \varphi_{i} \varphi_{j}+\alpha \sum_{j=1}^{n} \varphi_{j} V_{j}\right)-\frac{\alpha}{2 z}\left(\beta_{z}-\Delta \beta\right)-\alpha_{t} z \\
& +\frac{n}{2 t^{2}}-2 \sqrt{n} c_{t}-\frac{\alpha}{z} \sum_{j=1}^{n} \varphi_{j} \beta_{j}+\frac{\alpha}{2 z} \sum_{j=1}^{n} f_{j} \beta_{j} \\
& \leq \sqrt{n}\left[\sum_{j=1}^{n}\left(\triangle f_{j}\right)^{2}\right]^{\frac{1}{2}} z+\Delta V+\frac{1}{4}\left(2-\frac{\alpha}{z}\right)^{-1} \sum_{i, j=1}^{n}\left(f_{i, j}+f_{j, i}\right)^{2}+\frac{\lambda \alpha|\nabla \varphi|^{2}}{z} \\
& +\frac{\alpha}{z}|\nabla \varphi \| \nabla V|-\frac{\alpha\left(\beta_{t}-\Delta \beta\right)}{2 z}-\alpha_{t} z+\frac{n}{2 t^{2}}-2 \sqrt{n} c_{t} \\
& +\frac{\alpha}{z}|\nabla \varphi||\nabla \beta|+\frac{\alpha}{2 z}|f||\nabla \beta| \\
& \leq \sqrt{n}\left[\sum_{j=1}^{n}\left(\triangle f_{j}\right)^{2}\right]^{\frac{1}{2}} z+\Delta V+\frac{1}{6} \sum_{i, j=1}^{n}\left(f_{i, j}+f_{j, i}\right)^{2}+\lambda \alpha z+\frac{\alpha|\nabla \varphi|}{2 \sqrt{\beta}} \\
& -\frac{\alpha\left(\beta_{t}-\triangle \beta\right)}{2 z}-\alpha^{\prime}(t) z+\frac{n}{2 t^{2}}-2 \sqrt{n} c^{\prime}(t)+\frac{c_{1}(t)}{2} \alpha z \\
& +\frac{\alpha z}{2} \frac{|f||\nabla \beta|}{|\nabla \varphi|^{2}+\beta} \\
& \leq \sqrt{n}|\triangle f| z+\Delta V+\frac{1}{6} \sum_{i, j=1}^{n}\left(f_{i, i}+f_{j, i}\right)^{2}+\lambda \alpha z+\frac{\alpha|\nabla V|}{2 \sqrt{\beta}} \\
& +\frac{\alpha}{2 z}\left(\beta_{t}-\triangle \beta\right)-\alpha^{\prime}(t) z+\frac{n}{2 t^{2}}-2 \sqrt{n} c^{\prime}(t)+\frac{c_{1}(t)}{2} \alpha z \\
& +\frac{c_{1}(t)^{3 / 2}}{2} \alpha z
\end{aligned}
$$

Putting the upper estimate of R.H.S. of (2.16) and lower estimate of L.H.S. of (2.16) together, we have

$$
\begin{aligned}
& \frac{3 \alpha^{2} z^{2}}{2 n}+\frac{3 \alpha z}{2 t}-\frac{n \alpha}{4 t^{2} z}+\frac{3}{8} c^{2}+\frac{3}{2 \sqrt{n}} \alpha z c+\frac{3 \sqrt{n}}{4} \frac{c}{t} \\
\leq & \sqrt{n}|\triangle f| z+\triangle V+\frac{1}{6} \sum_{i, j=1}^{n}\left(f_{i, j}+f_{j, i}\right)^{2}+\lambda \alpha z+\frac{\alpha|\nabla V|}{2 \sqrt{\beta}} \\
& -\frac{\alpha}{2 z}\left(\beta_{t}-\triangle \beta\right)-\alpha^{\prime}(t) z+\frac{c_{1}+c_{1}^{3 / 2}}{2} \alpha z-2 \sqrt{n} c^{\prime}(t)
\end{aligned}
$$

(2.17) can be rewritten in the following form

$$
\begin{aligned}
& \frac{3 \alpha^{2}}{2 n} z^{2}+\left(\alpha^{\prime}(t) z+\frac{3 \alpha z c}{2 \sqrt{n}}-\lambda \alpha z-\sqrt{n}|\triangle f| z-\frac{\alpha|\nabla V|}{2 \sqrt{\beta}}-\frac{c_{1}+c_{1}^{3 / 2}}{2} \alpha z\right) \\
& +\left(2 \sqrt{n} c^{\prime}(t)+\frac{3}{8} c^{2}-\triangle V-\frac{1}{6} \sum_{i, j=1}^{n}\left(f_{i, j}+f_{j, i}\right)^{2}+\frac{3 \sqrt{n}}{4} \frac{c}{t}-\frac{\alpha \triangle \beta}{2 z}\right)
\end{aligned}
$$




$$
+\left(\frac{3 \alpha z}{2 t}+\frac{\alpha \beta_{t}}{2 z}-\frac{n \alpha}{4 t^{2} z}\right) \leq 0
$$

The above inequality implies

$$
\begin{aligned}
& \frac{3 \alpha^{2}}{2 n} z^{2}+z\left(\alpha^{\prime}(t)+\frac{3 \alpha c}{2 \sqrt{n}}-\lambda \alpha-\sqrt{n}|\Delta f|-\frac{\alpha|\nabla V|}{2 \sqrt{\beta}}-\frac{c_{1}+c_{1}^{3 / 2}}{2} \alpha\right) \\
+ & \left(2 \sqrt{n} c^{\prime}(t)\right)+\frac{3}{8} c^{2}-\Delta V-\frac{1}{6} \sum_{i, j=1}^{n}\left(f_{i, j}+f_{j, i}\right)^{2}+\frac{3 \sqrt{n}}{4} \frac{c}{t}-\frac{\alpha \Delta \beta}{\sqrt{\beta}} \\
+ & \frac{1}{z}\left(\frac{3 \alpha \beta}{2 t}+\frac{\alpha \beta_{t}}{2}-\frac{n \alpha}{4 t^{2}}\right) \leq 0
\end{aligned}
$$

However (2.18) contradicts with (2.3), (2.7), (2.8) and (2.9). Therefore we conclude that $\Psi$ cannot have an interior maximum with $\Psi=0$ at $t=t_{0}$.

LEMMA 2.1. Let $u_{\Omega}>0$ be a positive solution of the equation (2.1) defined on a compact domain $\Omega=\left\{x \in \mathbb{R}^{n}: \theta(x) \leq 0\right\}$ with the Neumann condition

$$
\frac{\partial u_{\Omega}}{\partial \nu}=0 \quad \text { on } \quad \partial \Omega
$$

where $\nu=\nabla \theta$ is an outward normal of $\partial \Omega$. Let $\varphi(x, t)=-\log u_{\Omega}(x, t)$ and $\Psi(x, t)$ be defined in (2.2). If $\frac{\partial \Psi}{\partial \nu}\left(x_{0}, t_{0}\right) \geq 0$ for $x_{0} \in \partial \Omega$, then at $\left(x_{0}, t_{0}\right)$

$$
\begin{aligned}
\frac{\partial V}{\partial \nu} \leq & -2\left(1-\frac{\alpha(t)}{2 \sqrt{|\nabla \varphi|^{2}+\beta}}\right) \sum_{i, j=1}^{n} \theta_{i j} \varphi_{i} \varphi_{j}-\sum_{i, j=1}^{n} \theta_{i} \frac{\partial f_{j}}{\partial x_{i}} \varphi_{j} \\
& +\sum_{i, j=1}^{n} \theta_{i j} f_{j} \varphi_{i}-\frac{\alpha(t) \sum_{i=1}^{n} \theta_{i} \beta_{i}}{2 \sqrt{|\nabla \varphi|^{2}+\beta}}
\end{aligned}
$$

Proof. Since

$$
\frac{\partial \varphi}{\partial \nu}=-\frac{\partial}{\partial \nu}(\log u)=-\frac{1}{u} \frac{\partial u}{\partial \nu}=0 \quad \text { on } \quad \partial \Omega
$$

we have $\frac{\partial \varphi_{t}}{\partial \nu}=0$ on $\partial \Omega$. On the other hand,

$$
\begin{aligned}
& \frac{\partial \varphi}{\partial \nu}=0 \quad \text { on } \quad \partial \Omega \\
\Longleftrightarrow & \sum_{i=1}^{n} \frac{\partial \theta}{\partial x_{i}} \frac{\partial \varphi}{\partial x_{i}}=0 \quad \text { on } \quad \partial \Omega \\
\Longrightarrow & \sum_{i=1}^{n} \theta_{i j} \varphi_{i}+\sum_{i=1}^{n} \theta_{i} \varphi_{i j}=0 \quad \text { on } \quad \partial \Omega \quad \text { for all } \quad 1 \leq j \leq n .
\end{aligned}
$$

Observe that

$$
\begin{aligned}
\frac{\partial}{\partial \nu}\left(\sum_{i=1}^{n} \varphi_{i}^{2}\right) & =\sum_{j=1}^{n} \frac{\partial \theta}{\partial x_{j}} \frac{\partial}{\partial x_{j}}\left(\sum_{i=1}^{n} \varphi_{i}^{2}\right)=2 \sum_{i=1}^{n} \sum_{j=1}^{n} \theta_{j} \varphi_{i} \varphi_{i j} \\
& =-2 \sum_{i=1}^{n} \sum_{j=1}^{n} \theta_{i j} \varphi_{i} \varphi_{j}
\end{aligned}
$$


Therefore, at $\left(x_{0}, t_{0}\right), x_{0} \in \partial \Omega$, we have

$$
\begin{gathered}
0 \leq \frac{\partial}{\partial \nu}\left[\varphi_{t}+|\nabla \varphi|^{2}-\sum_{j=1}^{2} f_{j} \varphi_{j}-V-\alpha(t) \sqrt{|\nabla \varphi|^{2}+\beta}-\frac{n}{2 t}-2 \sqrt{n} c(t)\right] \\
=-2 \sum_{i, j=1}^{n} \theta_{i j} \varphi_{i} \varphi_{j}-\sum_{i, j=1}^{n} \theta_{i} \frac{\partial f_{j}}{\partial x_{i}} \varphi_{j}-\sum_{i, j=1}^{n} \theta_{i} f_{j} \varphi_{j i}-\frac{\partial V}{\partial \nu} \\
-\frac{\alpha(t)\left[-2 \sum_{i, j=1}^{n} \theta_{i j} \varphi_{i} \varphi_{j}+\sum_{i=1}^{n} \theta_{i} \beta_{i}\right]}{2 \sqrt{|\nabla \varphi|^{2}+\beta}} \\
=-2\left(1-\frac{\alpha(t)}{2 \sqrt{|\nabla \varphi|^{2}+\beta}}\right) \sum_{i, j=1}^{n} \theta_{i j} \varphi_{i} \varphi_{j}-\sum_{i, j=1}^{n} \theta_{i} \frac{\partial f_{j}}{\partial x_{i}} \varphi_{j}+\sum_{i, j=1}^{n} \theta_{i j} f_{j} \varphi_{i} \\
-\frac{\partial V}{\partial \nu}-\frac{\alpha(t) \sum_{i=1}^{n} \theta_{i} \beta_{i}}{2 \sqrt{|\nabla \varphi|^{2}+\beta}}
\end{gathered}
$$

(2.19) follows immediately from the above inequality. $\square$

LEMMA 2.2. Let $u_{R}>0$ be a positive solution of the equation (2.1) defined on the closed ball $B_{R}=\left\{x \in \mathbb{R}^{n}: \theta(x)=|x|-R \leq 0\right\}$ with the Neumann condition

$$
\frac{\partial u_{R}}{\partial \nu}=0 \quad \text { on } \quad \partial B_{R} \quad \text { where } \nu=\nabla \theta
$$

Suppose $|f| \leq c(t)(1+|x|),|\nabla f|=\sqrt{\sum_{i=1}^{n}\left|\nabla f_{i}\right|^{2}} \leq c(t), 0<\alpha(t) \leq \frac{1}{2} \sqrt{\beta(x, t)}$ and $|\nabla \beta| \leq c_{1}(t) \sqrt{\beta(x, t)}$. Let $\varphi(x, t)=-\log u_{R}(x, t)$ and $\Psi(x, t)$ be defined in (2.2). If $\frac{\partial \Psi}{\partial \nu} \geq 0$ at $\left(x_{0}, t_{0}\right)$ with $x_{0} \in \partial B_{R}$, then

$$
\frac{\partial V}{\partial \nu} \leq \frac{R}{6}\left(1+\frac{\sqrt{n-1}}{R}\right)^{2}+\frac{\alpha(t)}{2} c_{1}(t) \text { at }\left(x_{0}, t_{0}\right)
$$

Proof. Since $\theta=|x|-R$, we have

$$
\theta_{i}=\frac{x_{i}}{|x|}, \nu=\left(\theta_{1}, \cdots, \theta_{n}\right) \text { and } \theta_{i j}=\frac{\delta_{i j}}{|x|}-\frac{x_{i} x_{j}}{|x|^{3}}
$$

The last equality implies

$$
\begin{array}{rlrl}
\sum_{i, j=1}^{n} \theta_{i j} \varphi_{i} \varphi_{j} & =\frac{1}{R}|\nabla \varphi|^{2}-\frac{\left(\sum_{i-1}^{n} x_{i} \varphi_{i}\right)^{2}}{R^{3}} & \text { on } \partial B_{R} \\
& =\frac{1}{R}|\nabla \varphi|^{2}-\frac{1}{R}\left|\frac{\partial \varphi}{\partial \nu}\right|^{2} & & \text { on } \partial B_{R} \\
& =\frac{1}{R}|\nabla \varphi| & \text { on } \partial B_{R}
\end{array}
$$


Observe that

$$
\begin{aligned}
& \left|\sum_{j=1}^{n}\left(\sum_{i=1}^{n} \theta_{i} \frac{\partial f_{j}}{\partial x_{i}}\right) \varphi_{j}\right| \leq \sqrt{\sum_{j=1}^{n}\left(\sum_{i=1}^{n} \theta_{i} \frac{\partial f_{j}}{\partial x_{i}}\right)^{2}}|\nabla \varphi| \\
& \leq \sqrt{\sum_{j=1}^{n}\left(\sum_{i=1}^{n} \theta_{i}^{2}\right)\left(\sum_{i=1}^{n}\left(\frac{\partial f_{j}}{\partial x_{i}}\right)^{2}\right)}|\nabla \varphi| \\
& =\sqrt{\sum_{j=1}^{n}\left|\nabla f_{j}\right|^{2}}|\nabla \varphi|=|\nabla f||\nabla \varphi| \\
& \leq c(t)|\nabla \varphi| \\
& \left|\sum_{i, j=1}^{n} \theta_{i j} f_{j} \varphi_{i}\right| \leq \sqrt{\sum_{i=1}^{n}\left(\sum_{j=1}^{n} \theta_{i j} f_{j}\right)^{2}}|\nabla \varphi| \\
& \leq \sqrt{\sum_{i=1}^{n}\left(\sum_{j=1}^{n} \theta_{i j}^{2}\right)\left(\sum_{j=1}^{n} f_{j}^{2}\right)}|\nabla \varphi| \\
& =\sqrt{\sum_{i, j=1}^{n} \theta_{i, j}^{2}}|\nabla f||\nabla \varphi| \\
& =\left[\sum_{i, j=1}^{n}\left(\frac{\delta_{i j}^{2}}{|x|^{2}}+\sum_{i, j=1}^{n} \frac{x_{i}^{2} x_{j}^{2}}{|x|^{6}}-2 \sum_{i, j=1}^{n} \frac{\delta_{i j} x_{i} x_{j}}{|x|^{4}}\right)\right]^{\frac{1}{2}}|\nabla f||\nabla \varphi| \\
& =\frac{\sqrt{n-1}}{|x|}|\nabla f||\nabla \varphi| \leq \frac{c \sqrt{n-1}}{R}|\nabla \varphi| \text { on } \partial B_{R} \\
& \frac{\alpha}{2 \sqrt{|\nabla \varphi|+\beta}} \leq \frac{\alpha}{2 \sqrt{\beta}} \leq \frac{1}{4} \\
& -2\left(1-\frac{\alpha}{2 \sqrt{|\nabla \varphi|+\beta}}\right) \leq-\frac{3}{2} \\
& \frac{-\alpha \sum_{i=1}^{n} \theta_{i} \beta_{i}}{2 \sqrt{|\nabla \varphi|^{2}+\beta}} \leq \frac{\alpha}{2} \frac{|\nabla \theta||\nabla \beta|}{\sqrt{|\nabla \varphi|^{2}+\beta}}=\frac{\alpha}{2} \frac{|\nabla \beta|}{\sqrt{|\nabla \varphi|^{2}+\beta}} \\
& \leq \frac{\alpha}{2} \frac{|\nabla \beta|}{\sqrt{\beta}} \leq \frac{\alpha}{2} c_{1}(t)
\end{aligned}
$$

Therefore (2.19) implies

$$
\begin{aligned}
\frac{\partial V}{\partial \nu} \leq- & \frac{3}{2 R}|\nabla \varphi|^{2}+c(t)\left(1+\frac{\sqrt{n-1}}{R}\right)|\nabla \varphi|+\frac{\alpha}{2} c_{1}(t) \\
=- & \frac{3}{2 R}\left[|\nabla \varphi|^{2}-\frac{2 R}{3}\left(1+\frac{\sqrt{n-1}}{R}\right)|\nabla \varphi|+\frac{R^{2}}{9}\left(1+\frac{\sqrt{n-1}}{R}\right)^{2}\right] \\
& \quad+\frac{R}{6}\left(1+\frac{\sqrt{n-1}}{R}\right)^{2}+\frac{\alpha}{2} c_{1}(t)
\end{aligned}
$$




$$
\begin{aligned}
& =-\frac{3}{2 R}\left[|\nabla \varphi|-\frac{R}{3}\left(1+\frac{\sqrt{n-1}}{R}\right)\right]^{2}+\frac{R}{6}\left(1+\frac{\sqrt{n-1}}{R}\right)^{2}+\frac{\alpha}{2} c_{1}(t) \\
& \leq \frac{R}{6}\left(1+\frac{\sqrt{n-1}}{R}\right)^{2}+\frac{\alpha(t)}{2} c_{1}(t)
\end{aligned}
$$

THEOREM 2.2. Let $u_{R}>0$ be a positive solution of the equation

$$
\frac{\partial u}{\partial t}=\Delta u+\sum_{i=1}^{n} f_{i} u_{i}-V u
$$

defined on the closed ball $B_{R}=\left\{x \in \mathbb{R}^{n}: \theta(x)=|x|-R \leq 0\right\}$, wth the Neumann condition

$$
\frac{\partial u_{R}}{\partial \nu}=0 \quad \text { on } \quad \partial B_{R} \quad \text { where } \quad \nu=\nabla \theta
$$

Let $\varphi(x, t)=-\log u_{R}(x, t)$ and

$$
\Psi(x, t)=\varphi_{t}+|\nabla \varphi|^{2}-\sum_{j=1}^{n} f_{j} \frac{\partial \varphi}{\partial x_{j}}-V-\alpha(t) \sqrt{|\nabla \varphi|^{2}+\beta(x, t)}-\frac{n}{2 t}-2 \sqrt{n} c(t)
$$

Suppose

$$
\begin{aligned}
& |f|^{2} \leq c(t)^{2}(1+|x|)^{2} \leq c_{1}(t) \beta(x, t) \\
& |\nabla f|=\sqrt{\sum_{i=1}^{n}\left|\nabla f_{i}\right|^{2} \leq c(t)} \\
& 0<\alpha(t) \leq \frac{1}{2} \sqrt{\beta(x, t)} \\
& |\nabla \beta| \leq c_{1}(t) \sqrt{\beta(x, t)} \\
& \frac{\beta_{t}}{2}+\frac{3 \beta}{2 t}-\frac{n}{4 t^{2}}>0 \\
& \alpha^{\prime}(t)+\alpha\left(\frac{3 c}{2 \sqrt{n}}-\lambda-\frac{|\nabla V|}{2 \sqrt{\beta}}-\frac{1}{2}\left(c_{1}+c_{1}\right)^{3 / 2}\right)-\sqrt{n}|\nabla f| \geq 0 \\
& c^{\prime}(t)+\frac{3}{16 \sqrt{n}} c^{2}+\frac{3}{8 t} c-\frac{1}{2 \sqrt{n}} \Delta V-\frac{1}{12 \sqrt{n}} \sum_{i, j=1}^{n}\left(\frac{f_{i, j}+f_{j, i}}{2}\right)^{2} \\
& \quad-\frac{\alpha \Delta \beta}{2 \sqrt{n} \sqrt{\beta}} \geq 0
\end{aligned}
$$

where $\lambda$ is the absolute value of the greatest eigenvalue of $\left(\frac{f_{i, j}+f_{j, i}}{2}\right)$.

$$
\frac{\partial V}{\partial \nu}>\frac{R}{6}\left(1+\frac{\sqrt{n-1}}{R}\right)^{2}+\frac{\alpha(t)}{2} c_{1}(t) \text { on } \partial B_{R} \text { for } t>0
$$

If $\Psi(x, 0)<0$, then $\Psi(x, t)<0$ for all $t>0$, i.e.

$\varphi_{t}+|\nabla \varphi|^{2}-\sum_{j=1}^{n} f_{j} \frac{\partial \varphi}{\partial x_{j}}-V<\alpha(t) \sqrt{|\nabla \varphi|^{2}+\beta(x, t)}+\frac{n}{2 t}+2 \sqrt{n} c(t)$ for all $t \geq 0$. 
Proof. If the conclusion is not true, then there exists $t_{0}$ such that $\Psi<0$ for $0 \leq t<t_{0}$ and $\Psi=0$ at some point $x_{0}$ when $t=t_{0}$. In view of the hypothesis (2.22)-(2.28), we can apply Theorem 2.1 to conclude that $x_{0} \in \partial B_{R}$. It is clear that $\frac{\partial \Psi}{\partial \nu}\left(x_{0}, t_{0}\right) \geq 0$. In view of Lemma 2.2 , we hare inequality $(2.20)$ which contradicts to our assumptions (2.29).

THEOREM 2.3. Let $u_{R}>0$ be a positive solution of the equation

$$
\frac{\partial u}{\partial t}=\Delta u+\sum_{i=1}^{n} f_{i} u_{i}-V u
$$

defined on the local ball $B_{R}=\left\{x \in \mathbb{R}^{n}: \theta(x)=|x|-R \leq 0\right\}$ with the Neumann condition

$$
\frac{\partial u_{R}}{\partial \nu}=0 \quad \text { on } \quad \partial B_{R} \quad \text { where } \quad \nu=\nabla \theta
$$

Let $\varphi(x, t)=-\log u_{R}(x, t)$ and

$$
\Psi(x, t)=\varphi_{t}+|\nabla \varphi|^{2}-\sum_{j=1}^{n} f_{j} \frac{\partial \varphi}{\partial x_{j}}-V-\alpha(t) \sqrt{|\nabla \varphi|^{2}+\beta(x, t)}-\frac{n}{2 t}-2 \sqrt{n} c(t)
$$

Let $\beta(x, t)=c_{2}(t)|x|^{2}+4 \alpha^{2}(t)+\frac{n}{2 t}$ with $c_{2}(t)>0$ and $\alpha(t)>0$. Suppose that there exists $c_{1}(t)>0$ such that

$$
\begin{aligned}
& |f| \leq c(t)(1+|x|) \\
& |\nabla f|=\sqrt{\sum_{i=1}^{n}\left|\nabla f_{i}\right|^{2} \leq c(t),|\nabla V| \leq c(1+|x|)} \\
& |\triangle V| \leq c,|\triangle f| \leq c \\
& 2 c^{2}(t) \leq c_{1}(t) c_{2}(t) \\
& 2 c^{2}(t) \leq c_{1}(t)\left(4 \alpha^{2}(t)+\frac{n}{2 t}\right) \\
& 4 c_{2}(t) \leq c_{1}^{2}(t) \\
& c_{2}^{\prime}+\frac{3}{t} c_{2} \geq 0 \\
& 2\left(\alpha^{2}\right)^{\prime}+\frac{6}{t} \alpha^{2}+\frac{n}{4 t^{2}}>0 \\
& \alpha^{\prime}(t)+\alpha\left[\frac{3 c}{2 \sqrt{n}}-\lambda-\frac{1}{2}\left(\sqrt{c_{1}}+c_{1}+c_{1}^{3 / 2}\right]-\sqrt{n} c \geq 0\right. \\
& c^{\prime}(t)+\frac{5}{48 \sqrt{n}} c^{2}+\left(\frac{3}{8 t}-\frac{1}{2 \sqrt{n}}\right) c-\frac{\sqrt{n}}{2} c_{2}>0
\end{aligned}
$$

where $\lambda$ is the absolute value of the greatest eigenvlaue of $\left(\frac{f_{i, j}+f_{j, i}}{2}\right)$

$$
\frac{\partial V}{\partial \nu}>\frac{R}{6}\left(1+\frac{\sqrt{n-1}}{R}\right)^{2}+\frac{\alpha(t)}{2} c_{1}(t) \quad \text { on } \quad \partial B_{R} \text { for } \quad t>0
$$


If $\Psi(x, 0)<0$, then $\Psi(x, t)<0$ for all $t>0$, i.e.,

$$
\begin{gathered}
\varphi_{t}+|\nabla \varphi|^{2}-\sum_{j=1}^{n} f_{j} \frac{\partial \varphi}{\partial x_{j}}-V \\
<\alpha(t) \sqrt{|\nabla \varphi|^{2}+\beta(x, t)}+\frac{n}{2 t}+2 \sqrt{n} c(t) \text { for all } t \geq 0 .
\end{gathered}
$$

Proof. We only need to prove that (2.31)-(2.40) imply (2.22)-(2.28).

Observe that

$$
\begin{aligned}
c(t)^{2}(1+|x|)^{2} & \leq 2 c(t)^{2}\left(1+|x|^{2}\right) \\
& \leq c_{1}(t) c_{2}(t)|x|^{2}+\left(\frac{n}{2 t}+4 \alpha^{2}(t)\right) c_{1}(t)=c_{1}(t)=c_{1}(t) \beta(x, t)
\end{aligned}
$$

by (2.34) and (2.35). Hence (2.22) follows. (2.23) is part of our assumption (2.32) while (2.24) follows from our definition of $\beta(x, t)$ and the hypothesis $c_{2}(t)>0$.

(2.25) is equivalent to $2 c_{2}|x| \leq c_{1} \sqrt{c_{2}|x|^{2}+4 \alpha^{2}+\frac{n}{2 t}}$, i.e., $4 c_{2}^{2}|x|^{2} \leq c_{1}^{2}\left(c_{2}|x|^{2}+\right.$ $\left.4 \alpha^{2}+\frac{n}{2 t}\right)$. Hence (2.36) implies (2.25)

$$
\begin{aligned}
\frac{\beta_{t}}{2}+\frac{3 \beta}{2 t}-\frac{n}{4 t^{2}} & =\frac{1}{2}\left(c_{2}^{\prime}|x|^{2}+8 \alpha \alpha^{\prime}-\frac{n}{2 t^{2}}\right)+\frac{3 c_{2}}{2 t}|x|^{2}+\frac{6 \alpha^{2}}{t}+\frac{3 n}{4 t^{2}}-\frac{n}{4 t^{2}} \\
& =\left(\frac{1}{2} c_{2}^{\prime}+\frac{3}{2 t} c_{2}\right)|x|^{2}+4 \alpha^{\prime} \alpha+\frac{6}{t} \alpha^{2}+\frac{n}{4 t^{2}}
\end{aligned}
$$

Therefore (2.37) and (2.38) imply (2.26)

$$
\begin{aligned}
& \alpha^{\prime}(t)+\alpha\left[\frac{3 c}{2 \sqrt{n}}-\lambda-\frac{|\nabla V|}{2 \sqrt{\beta}}-\frac{1}{2}\left(c_{1}+c_{1}^{3 / 2}\right)\right]-\sqrt{n}|\Delta f| \\
\geq & \alpha^{\prime}(t)+\alpha\left[\frac{3 c}{2 \sqrt{n}}-\lambda-\frac{c(1+|x|)}{2 \sqrt{\beta}}-\frac{1}{2}\left(c_{1}+c_{1}^{3 / 2}\right)\right]-\sqrt{n} c \\
\geq & \alpha^{\prime}(t)+\alpha\left[\frac{3 c}{2 \sqrt{n}}-\lambda-\frac{1}{2}\left(\sqrt{c_{1}}+c_{1}+c_{1}^{3 / 2}\right)\right]-\sqrt{n} c
\end{aligned}
$$

Hence (2.39) implies (2.27).

Observe that

$$
\begin{aligned}
& \sum_{i, j=1}^{n}\left(\frac{f_{i, j}+f_{j, i}}{2}\right)^{2}=\frac{1}{2} \sum_{i, j=1}^{n} f_{i, j}^{2}+\frac{1}{2} \sum_{i, j=1}^{n} f_{i, j} f_{j, i} \\
& \leq \frac{1}{2} \sum_{i, j=1}^{n} f_{i, j}^{2}+\frac{1}{4}\left(\sum_{i, j=1}^{n} f_{i, j}^{2}+\sum_{i, j=1}^{n} f_{j, i}^{2}\right) \\
&=\sum_{i, j=1}^{n} f_{i, j}^{2}=\sum_{i=1}^{n}\left|\nabla f_{i}\right|^{2}=|\nabla f|^{2} \leq c^{2} \\
& c^{\prime}(t)+\frac{3}{16 \sqrt{n}} c^{2}+\frac{3}{8 t} c-\frac{1}{2 \sqrt{n}} \Delta V-\frac{1}{12 \sqrt{n}} \sum_{i, j=1}^{n}\left(\frac{f_{i, j}+f_{j, i}}{2}\right)^{2}-\frac{\alpha \Delta \beta}{2 \sqrt{n} \sqrt{\beta}} \\
& \geq c^{\prime}(t)+\frac{3}{16 \sqrt{n}} c^{2}+\frac{3}{8 t} c-\frac{1}{2 \sqrt{n}} c(t)-\frac{1}{12 \sqrt{n}} c^{2}-\frac{2 n c_{2}}{2 \sqrt{n}} \cdot \frac{1}{2} \\
&=c^{\prime}(t)+\frac{5}{48 \sqrt{n}} c^{2}+\left(\frac{3}{8 t}-\frac{1}{2 \sqrt{n}}\right) c-\frac{\sqrt{n}}{2} c_{2}
\end{aligned}
$$


(2.28) follows immediately from (2.40).

THEOREM 2.4. Let $u>0$ be a positive solution of the equation

$$
\frac{\partial u}{\partial t}=\Delta u+\sum_{i=1}^{n} f_{i} u_{i}+V u
$$

on $\mathbb{R}^{n}$. Let $\varphi(x, t)=-\log u(x, t)$ and

$$
\Psi(x, t)=\varphi_{t}+|\nabla \varphi|^{2}-\sum_{j=1}^{n} f_{j} \frac{\partial \varphi}{\partial x_{j}}-V-\alpha(t) \sqrt{|\nabla \varphi|^{2}+\beta(x, t)}-\frac{n}{2 t}-2 \sqrt{n} c(t)
$$

Let $\beta(x, t)=c_{2}(t)|x|^{2}+4 \alpha^{2}(t)+\frac{n}{2 t}$ with $c_{2}(t)>0$ and $\alpha(t)>0$. Suppose that there exists $c_{1}(t)>0$ such that

$$
\begin{aligned}
& |f| \leq c(t)(1+|x|) \\
& |\nabla f|=\sqrt{\sum_{i=1}^{n}\left|\nabla f_{i}\right|^{2}} \leq c(t),|\nabla V| \leq c(1+|x|) \\
& |\Delta V| \leq c,|\triangle f| \leq c \\
& 2 c^{2}(t) \leq c_{1}(t) c_{2}(t) \\
& 2 c^{2}(t) \leq c_{1}(t)\left(4 \alpha^{2}(t)+\frac{n}{2 t}\right) \\
& 4 c_{2}(t) \leq c_{1}^{2}(t) \\
& c_{2}^{\prime}(t)+\frac{3}{t} c_{2} \geq 0 \\
& 2\left(\alpha^{2}\right)^{\prime}+\frac{6}{t} \alpha^{2}+\frac{n}{4 t^{2}}>0 \\
& \alpha^{\prime}(t)+\alpha\left[\frac{3 c}{2 \sqrt{n}}-\lambda-\frac{1}{2}\left(\sqrt{c_{1}}+c_{1}+c_{1}^{3 / 2}\right)\right]-\sqrt{n} c \geq 0 \\
& c^{\prime}(t)+\frac{5}{48 \sqrt{n}} c^{2}+\left(\frac{3}{8 t}-\frac{1}{2 \sqrt{n}}\right) c-\frac{\sqrt{n}}{2} c_{2}>0
\end{aligned}
$$

where $\lambda$ is the absolute value of the greatest eigenvalue of $\left(\frac{f_{i, j}+f_{j, i}}{2}\right)$. If $\Psi(x, 0)<0$, then $\Psi(x, t)<0$ for all $t>0$, i.e.

$$
\begin{aligned}
& \triangle \varphi=\varphi_{t}+|\nabla \varphi|^{2}-\sum_{j=1}^{n} f_{j} \frac{\partial \varphi}{\partial x_{j}}-V \\
& <\alpha(t) \sqrt{|\nabla \varphi|^{2}+\beta(x, t)}+\frac{n}{2 t}+2 \sqrt{n} c(t) \text { for all } t \geq 0 .
\end{aligned}
$$

Proof. Choose a function $A_{R}(x, t)$ which is convex in $x$ direction with the following properties.

$$
\begin{aligned}
& A_{R}(x, t) \equiv 0 \text { for }|x| \leq R / 2 \\
& \left|\nabla V_{R}\right| \leq c(1+|x|),\left|\triangle V_{R}\right| \leq c \\
& \frac{\partial V_{R}}{\partial \nu}>\frac{R}{6}\left(1+\frac{\sqrt{n-1}}{R}\right)^{2}+\frac{\alpha(t)}{2} c_{1}(t) \text { on } \partial B_{R} \text { for } t>0
\end{aligned}
$$


where $V_{R}=V+A_{R}$. Let $f_{R}=f \cdot \sigma$ where $\sigma$ is a cut off function with the following properties

$$
\begin{aligned}
& \sigma(x)=1 \text { for }|x| \leq \frac{R}{2} \text { and } \sigma(x)=0 \text { for }|x| \geq R \\
& \left|\nabla f_{R}\right| \leq c,|\triangle f| \leq c
\end{aligned}
$$

Let $u_{R}$ be the positive solution of the equation

$$
\frac{\partial u_{R}}{\partial t}=\Delta u_{R}+\sum_{i=1}^{n}\left(f_{R}\right)_{i}\left(u_{R}\right)_{i}-V_{R} u_{R}
$$

defined on the closed ball $B_{R}=\left\{x \in \mathbb{R}^{n}: \theta(x)=|x|-R \leq 0\right\}$ with the Neumann condition $\frac{\partial u_{R}}{\partial \nu}=0$ on $\partial B_{R}$ where $\nu=\nabla \theta$. In view of Theorem 2.3, we know that (2.42) holds for $u_{R}$. Since $u$ is the limit of $u_{R}$ as $R$ goes to infinity, we conclude that (2.53) holds.

Remark : It is easy to choose $c, c_{1}, c_{2}$ and $\alpha$ such that (2.43)-(2.52) are satisfied. For instance, we can take $c_{1}=2 c^{2 / 3}, c_{2}=c^{4 / 3}$ and $\alpha(t)=\frac{1}{T-t}$ where $c$ and $T$ are positive constant and $T$ is sufficiently small.

3. Harnack Inequality. In this section, we shall use the gradient estimate obtained in Theorem 2.3 to deduce Harnack inequality.

Proposition 3.1. Let $u_{R}>0$ be a positive solution of the equation

$$
\frac{\partial u}{\partial t}=\Delta u+\sum_{i=1}^{n} f_{i} \frac{\partial u}{\partial x_{i}}-V u
$$

defined on the closed ball $B_{R}=\left\{x \in \mathbb{R}^{n}: \theta(x)=|x|-R \leq 0\right\}$ with the Neumann condition

$$
\frac{\partial u_{R}}{\partial \nu}=0 \quad \text { on } \quad \partial B_{R} \quad \text { where } \quad \nu=\nabla \theta
$$

Let $\varphi(x, t)=-\log u_{R}(x, t)$ and $\beta(x, t)=c_{2}(t)|x|^{2}+4 \alpha^{2}(t)+n /(2 t)$ with $c_{2}(t)>0$ and $\alpha(t)>0$. Suppose that

$$
\varphi_{t}+|\nabla \varphi|^{2}-\sum_{j=1}^{n} f_{j} \frac{\partial \varphi}{\partial x_{j}}-V \leq \alpha(t) \sqrt{|\nabla \varphi|^{2}+\beta(x, t)}+\frac{n}{2 t}+2 \sqrt{n} c(t)
$$

Let $t>t_{0}$ and $\mathcal{P}=\left\{\right.$ differentiable path $\sigma=\left(\sigma_{1}, \sigma_{2}\right):[0,1] \longrightarrow B_{R} \times \mathbb{R}$ such that $\sigma(0)$ $\left.=\left(\sigma_{1}(0), \sigma_{2}(0)\right)=\left(x_{0}, t_{0}\right), \sigma(1)=(x, t), \sigma_{2}^{\prime}(s)>0\right\}$. Define

$$
\begin{aligned}
d\left(\left(x_{0}, t_{0}\right),(x, t)\right):=\inf _{\sigma \in \mathcal{P}} & \left\{\frac { 1 } { 2 } \int _ { 0 } ^ { 1 } \left\langle\dot{\sigma_{1}}, f>d s+\frac{1}{4} \int_{0}^{1} \frac{d \sigma_{2}}{d s}\left[\frac{\left|\dot{\sigma}_{1}\right|}{\frac{d \sigma_{2}}{d s}}+\sqrt{2} \alpha\right]^{2} d s\right.\right. \\
& \left.+\int_{0}^{1} \frac{d \sigma_{2}}{d s}\left(\sqrt{2} \alpha \sqrt{\frac{|f|^{2}}{4}+\frac{\beta}{2}}+V+\frac{1}{4} \sum_{i=1}^{n} f_{i}^{2}\right) d s\right\}
\end{aligned}
$$

Then

$$
\frac{u_{R}(x, t)}{u_{R}\left(x_{0}, t_{0}\right)} \geq\left(\frac{t}{t_{0}}\right)^{-\frac{n}{2}} \exp \left(-\int_{t_{0}}^{t} 2 \sqrt{n} c(\tau) d \tau\right) \exp \left[-d\left(\left(x_{0}, t_{0}\right),(x, t)\right)\right]
$$


Proof. We shall rewrite the inequality (3.1) in the following form

$$
\begin{aligned}
& \varphi_{t}+\left|\nabla \varphi-\frac{f}{2}\right|^{2}-\frac{|f|^{2}}{4}-V=\varphi_{t}+|\nabla \varphi|^{2}-\sum_{i=1}^{n} f_{i} \varphi_{i}-V \\
& \leq \alpha \sqrt{|\nabla \varphi|^{2}+\beta}+\frac{n}{2 t}+2 \sqrt{n} c(t) \\
& \leq \alpha \sqrt{\left(\left|\nabla \varphi-\frac{f}{2}\right|+\left|\frac{f}{2}\right|\right)^{2}+\beta}+\frac{n}{2 t}+2 \sqrt{n} c(t) \\
& \leq \alpha \sqrt{2\left|\nabla \varphi-\frac{f}{2}\right|^{2}+\frac{|f|^{2}}{2}+\beta}+\frac{n}{2 t}+2 \sqrt{n} c(t) \\
& =\alpha \sqrt{2} \sqrt{\left|\nabla \varphi-\frac{f}{2}\right|^{2}+\frac{|f|^{2}}{4}+\frac{\beta}{2}}+\frac{n}{2 t}+2 \sqrt{n} c(t) \\
& \leq \alpha \sqrt{2}\left(\left|\nabla \varphi-\frac{f}{2}\right|+\sqrt{\frac{|f|^{2}}{4}+\frac{\beta}{2}}\right)+\frac{n}{2 t}+2 \sqrt{n} c(t)
\end{aligned}
$$

Let $\sigma=\left(\sigma_{1}, \sigma_{2}\right)$ be a path in $\mathcal{P}$. Then

$$
\begin{aligned}
& =-\int_{0}^{1}\left\langle\dot{\sigma_{1}}, \nabla \varphi\right\rangle-\int_{0}^{1} \frac{d \sigma_{2}}{d s} \frac{\partial \varphi}{\partial t} \\
& \geq-\int_{0}^{1}\left\langle\dot{\sigma_{1}}, \nabla \varphi-\frac{f}{2}\right\rangle-\frac{1}{2} \int_{0}^{1}\left\langle\dot{\sigma_{1}}, f\right\rangle+\int_{0}^{1} \frac{d \sigma_{2}}{d s}\left(\left|\nabla \varphi-\frac{f}{2}\right|^{2}\right. \\
& \left.-\alpha \sqrt{2}\left|\nabla \varphi-\frac{f}{2}\right|-\alpha \sqrt{2} \sqrt{\frac{|f|^{2}}{4}+\frac{\beta}{2}}-\frac{|f|^{2}}{4}-V-\frac{n}{2 \sigma_{2}(s)}-2 \sqrt{n} c\left(\sigma_{2}(s)\right)\right) \\
& \geq-\frac{1}{2} \int_{0}^{1}\left\langle\dot{\sigma_{1}}, f>-\int_{0}^{1} \frac{d \sigma_{2}}{d s}\left(\alpha \sqrt{2} \sqrt{\frac{|f|^{2}}{4}+\frac{\beta}{2}}+\frac{|f|^{2}}{4}+V+\frac{n}{2 \sigma_{2}(s)}+2 \sqrt{n} c\left(\sigma_{2}(s)\right)\right)\right. \\
& +\int_{0}^{1} \frac{d \sigma_{2}}{d s}\left[\left|\nabla \varphi-\frac{f}{2}\right|^{2}-\left(\alpha \sqrt{2}+\frac{\left|\dot{\sigma_{1}}\right|}{\frac{d \sigma_{2}}{d s}}\right)\left|\nabla \varphi-\frac{f}{2}\right|\right] \\
& =-\frac{1}{2} \int_{0}^{1}\left\langle\dot{\sigma_{1}}, f>-\int_{0}^{1} \frac{d \sigma_{2}}{d s}\left[\alpha \sqrt{2} \sqrt{\frac{|f|^{2}}{4}+\frac{\beta}{2}}+\frac{|f|^{2}}{4}+V+\frac{n}{2 \sigma_{2}(s)}+2 \sqrt{n} c\left(\sigma_{2}(s)\right)\right]\right. \\
& -\frac{1}{4} \int_{0}^{1} \frac{d \sigma_{2}}{d s}\left(\alpha \sqrt{2}+\frac{\left|\dot{\sigma}_{1}\right|}{\frac{d \sigma_{2}}{d s}}\right)^{2} \\
& +\int_{0}^{1} \frac{d \sigma_{2}}{d s}\left[\left|\nabla \varphi-\frac{f}{2}\right|^{2}-\frac{1}{2}\left(\alpha \sqrt{2}+\frac{\left|\dot{\sigma}_{1}\right|}{\frac{d \sigma_{2}}{d s}}\right)\right]^{2} \\
& \geq-\frac{1}{2} \int_{0}^{1}\left\langle\dot{\sigma_{1}}, f>-\int_{0}^{1} \frac{d \sigma_{2}}{d s}\left[\alpha \sqrt{2} \sqrt{\frac{|f|^{2}}{4}+\frac{\beta}{2}}+\frac{|f|^{2}}{4}+V+\frac{n}{2 \sigma_{2}(s)}+2 \sqrt{n} c\left(\sigma_{2}(s)\right)\right]\right. \\
& -\frac{1}{4} \int_{0}^{1} \frac{d \sigma_{2}}{d s}\left(\alpha \sqrt{2}+\frac{\left|\dot{\sigma}_{1}\right|}{\frac{d \sigma_{2}}{d s}}\right)^{2} \\
& =-\frac{1}{2} \int_{0}^{1}<\dot{\sigma_{1}}, f>-\int_{0}^{1} \frac{d \sigma_{2}}{d s}\left[\alpha \sqrt{2} \sqrt{\frac{|f|^{2}}{4}+\frac{\beta}{2}}+\frac{|f|^{2}}{4}+V\right]
\end{aligned}
$$




$$
\begin{aligned}
& -\frac{1}{4} \int_{0}^{1} \frac{d \sigma_{2}}{d s}\left(\alpha \sqrt{2}+\frac{\left|\dot{\sigma}_{1}\right|}{\frac{d \sigma_{2}}{d s}}\right)^{2}-\int_{0}^{1} \frac{n}{2 \sigma_{2}(s)} d \sigma_{2}(s)-\int_{0}^{1} 2 \sqrt{n} c\left(\sigma_{2}(s)\right) d \sigma_{2}(s) \\
= & -\frac{1}{2} \int_{0}^{1}<\dot{\sigma_{1}}, f>-\int_{0}^{1} \frac{d \sigma_{2}}{d s}\left[\alpha \sqrt{2} \sqrt{\frac{|f|^{2}}{4}+\frac{\beta}{4}}+\frac{|f|^{2}}{4}+V\right] \\
& -\frac{1}{4} \int_{0}^{1} \frac{d \sigma_{2}}{d s}\left(\alpha \sqrt{2}+\frac{\left|\dot{\sigma_{1}}\right|}{\frac{d \sigma_{2}}{d s}}\right)^{2}-\frac{n}{2} \log \left(\frac{t}{t_{0}}\right)-\int_{t_{0}}^{t} 2 \sqrt{n} c(\tau) d \tau
\end{aligned}
$$

Since (3.7) is true for all $\sigma \in \mathcal{P}$, we have

$$
\begin{aligned}
& \log u(x, t)-\log u\left(x_{0}, t_{0}\right) \\
\geq & \sup _{\sigma \in \mathcal{P}}\left\{-\frac{1}{2} \int_{0}^{1}<\dot{\sigma_{1}}, f>-\int_{0}^{1} \frac{d \sigma_{2}}{d s}\left[\alpha \sqrt{2} \sqrt{\frac{|f|^{2}}{4}+\frac{\beta}{2}}+\frac{|f|^{2}}{4}+V\right]\right. \\
& \left.-\frac{1}{4} \int_{0}^{1} \frac{d \sigma_{2}}{d s}\left(\alpha \sqrt{2}+\frac{\left|\sigma_{1}\right|}{\frac{d \sigma_{2}}{d s}}\right)^{2}\right\}-\frac{n}{2} \log \left(\frac{t}{t_{0}}\right)-\int_{t_{0}}^{t} 2 \sqrt{n} c(\tau) d \tau \\
= & -\inf _{\sigma \in \mathcal{P}}\left\{\frac{1}{2} \int_{0}^{1}<\dot{\sigma_{1}}, f>+\int_{0}^{1} \frac{d \sigma_{2}}{d s}\left[\alpha \sqrt{2} \sqrt{\frac{|f|^{2}}{4}+\frac{\beta}{4}}+\frac{|f|^{2}}{4}+V\right]\right. \\
& +\frac{1}{4} \int_{0}^{1} \frac{d \sigma_{2}}{d s}\left(\alpha \sqrt{2}+\frac{\left|\sigma_{1}\right|}{\frac{d \sigma_{2}}{d s}}\right\}^{2}-\frac{n}{2} \log \left(\frac{t}{t_{0}}\right)-\int_{t_{0}}^{t} 2 \sqrt{n} c(\tau) d \tau \\
= & -d\left(\left(x_{0}, t_{0}\right),(x, t)\right)-\frac{n}{2} \log \left(\frac{t}{t_{0}}\right)-\int_{t_{0}}^{t} 2 \sqrt{n} c(\tau) d \tau
\end{aligned}
$$

It follows that

$$
\frac{u(x, t)}{u\left(x_{0}, t_{0}\right)} \geq\left(\frac{t}{t_{0}}\right)^{-\frac{n}{2}} \exp \left(-\int_{t_{0}}^{t} 2 \sqrt{n} c(\tau) d \tau\right) \exp \left[-d\left(\left(x_{0}, t_{0}\right),(x, t)\right)\right]
$$

Proposition 3.2. Let $d\left(\left(x_{0}, t_{0}\right),(x, t)\right)$ be defined by (3.4) in Proposition 3.1. Then

$$
\begin{aligned}
& +\frac{t-t_{0}}{2} \int_{0}^{1} \alpha^{2}\left((1-s) t_{0}+s t\right) d s \\
& \left.+\frac{1}{2} \int_{0}^{1}<x-x_{0}, f(1-s) x_{0}+s x,(1-s) t_{0}+s t\right)>d s \\
& +\left(t-t_{0}\right) \int_{0}^{1}\left\{\sqrt{2} \alpha(1-s) t_{0}+s t\right)\left[\frac{1}{4} \sum_{i=1}^{n} f_{i}^{2}\left((1-s) x_{0}+s x,(1-s) t_{0}+s t\right)\right. \\
& \left.+\frac{1}{2} \beta\left((1-s) x_{0}+s x,(1-s) t_{0}+s t\right)\right]^{\frac{1}{2}} \\
& \left.+\frac{1}{4} \sum_{i=1}^{n} f_{i}^{2}\left((1-s) x_{0}+s x,(1-s) t_{0}+s t\right)+V\left((1-s) x_{0}+s x,(1-s) t_{0}+s t\right)\right\} d s
\end{aligned}
$$


Proof. Let $\left(\sigma_{1}, \sigma_{2}\right)=\left((1-s) x_{0}+s x,(1-s) t_{0}+s t\right)$. Then $\dot{\sigma}_{1}=x-x_{0}$ and $\frac{d \sigma_{2}}{d s}=$ $t-t_{0}$. By the definition of $d\left(\left(x_{0}, t_{0}\right),(x, t)\right)$, we have

$$
\begin{aligned}
& d\left(\left(x_{0}, t_{0}\right),(x, t)\right) \leq \frac{1}{2} \int_{0}^{1}<x-x_{0}, f\left((1-s) x_{0}+s x,(1-s) t_{0}+s t\right)>d s \\
& +\int_{0}^{1}\left(t-t_{0}\right)\left[\sqrt{2} \alpha\left((1-s) t_{0}+s t\right)\right. \\
& \sqrt{\frac{\left.\mid f(1-s) x_{0}+s x,(1-s) t_{0}+s t\right)\left.\right|^{2}}{4}+\frac{\beta\left((1-s) x_{0}+s x,(1-s) t_{0}+s t\right)}{2}} \\
& \left.+\frac{\left|f\left((1-s) x_{0}+s x,(1-s) t_{0}+s t\right)\right|^{2}}{4}+V\left((1-s) x_{0}+s x,(1-s) t_{0}+s t\right)\right] d s \\
& +\frac{1}{4} \int_{0}^{1}\left(t-t_{0}\right)\left(\left(\sqrt{2} \alpha(1-s) t_{0}+s t\right)+\frac{\left|x-x_{0}\right|}{t-t_{0}}\right)^{2} d s \\
& \leq \frac{\left|x-x_{0}\right|^{2}}{4\left(t-t_{0}\right)}+\frac{\sqrt{2}}{2}\left|x-x_{0}\right| \int_{0}^{1} \alpha\left((1-s) t_{0}+s t\right) d s+\frac{t-t_{0}}{2} \int_{0}^{1} \alpha^{2}\left((1-s) t_{0}+s t\right) d s \\
& \frac{1}{2} \int_{0}^{1}<x-x_{0}, f\left((1-s) x_{0}+s x,(1-s) t_{0}+s t\right)>d s \\
& +\left(t-t_{0}\right) \int_{0}^{1}\left\{\frac{1}{4} \sum_{i=1}^{n} f_{i}^{2}\left((1-s) x_{0}+s x,(1-s) t_{0}+s t\right)\right. \\
& +V\left((1-s) x_{0}+s x,(1-s) t_{0}+s t\right) \\
& +\sqrt{2} \alpha\left((1-s) t_{0}+s t\right)\left[\frac{1}{4} \sum_{i=1}^{n} f_{i}^{2}\left((1-s) x_{0}+s x,(1-s) t_{0}+s t\right)\right. \\
& \left.\left.+\frac{1}{2} \beta\left((1-s) x_{0}+s x,(1-s) t_{0}+s t\right)\right]^{\frac{1}{2}}\right] d s
\end{aligned}
$$

Similarly we can use Theorem 2.4 to deduce Harnack inequality on $\mathbb{R}^{n}$.

THEOREM 3.3. Let $u>0$ be a positive solution of the equation

$$
\frac{\partial u}{\partial t}=\Delta u+\sum_{i=1}^{n} f_{i} u_{i}-V u
$$

on $\mathbb{R}^{n}$. Let $\varphi(x, t)=-\log u(x, t)$ and

$$
\Psi(x, t)=\varphi_{t}+|\nabla \varphi|^{2}-\sum_{j=1}^{n} f_{j} \frac{\partial \varphi}{\partial x_{j}}-V-\alpha(t) \sqrt{|\nabla \varphi|^{2}+\beta(x, t)}-\frac{n}{2 t}-2 \sqrt{n} c(t)
$$

Let $\beta(x, t)=c_{2}(t)|x|^{2}+4 \alpha^{2}(t)+\frac{n}{2 t}$ with $c_{2}(t)>0$ and $\alpha(t)>0$. Suppose that there exists $c_{1}(t)$ such that

$$
\begin{aligned}
& |f| \leq c(1+|x|) \\
& |\nabla f|=\sqrt{\sum_{i=1}^{n}\left|\nabla f_{i}\right|^{2}} \leq c,|\nabla V| \leq c(1+|x|)
\end{aligned}
$$




$$
\begin{aligned}
& |\triangle V| \leq c,|\triangle f| \leq c \\
& 2 c^{2}(t) \leq c_{1}(t) c_{2}(t) \\
& 2 c^{2}(t) \leq c_{1}(t)\left(4 \alpha^{2}(t)+\frac{n}{2 t}\right) \\
& 4 c_{2}(t) \leq c_{1}^{2}(t) \\
& c_{2}^{\prime}(t)+\frac{3}{t} c_{2} \geq 0 \\
& 2\left(\alpha^{2}\right)^{\prime}+\frac{6}{t} \alpha^{2}+\frac{n}{4 t^{2}}>0 \\
& \alpha^{\prime}(t)+\alpha\left[\frac{3 c}{2 \sqrt{n}}-\lambda-\frac{1}{2}\left(\sqrt{c_{1}}+c_{1}+c_{1}^{3 / 2}\right)\right]-\sqrt{n} c \geq 0 \\
& c^{\prime}(t)+\frac{5}{48 \sqrt{n}} c^{2}+\left(\frac{3}{8 t}-\frac{1}{2 \sqrt{n}}\right) c-\frac{n}{2} c_{2}>0
\end{aligned}
$$

where $\lambda$ is the absolute value of the greatest eigenvalue of $\left(\frac{f_{i, j}+f_{j, i}}{2}\right)$.

Let $t>t_{0}$ and $\mathcal{P}=\left\{\right.$ differentiable path $\sigma=\left(\sigma_{1}, \sigma_{2}\right):[0,1] \rightarrow \mathbb{R}^{n} \times \mathbb{R}$ such that $\left.\sigma(0)=\left(\sigma_{1}(0), \sigma_{2}(0)\right)=\left(x_{0}, t_{0}\right), \sigma(1)=(x, t), \sigma_{2}^{\prime}(s)>0\right\}$. Define

$$
\begin{aligned}
d\left(\left(x_{0}, t_{0}\right),(x, t)\right): & =\inf _{\sigma \in \mathcal{P}}\left\{\frac{1}{2} \int_{0}^{1}<\dot{\sigma}_{1}, f>d s+\int_{0}^{1} \frac{d \sigma_{2}}{d s}\left(\sqrt{2} \alpha \sqrt{\frac{|f|^{2}}{4}+\frac{\beta}{2}}\right.\right. \\
& \left.\left.+V+\frac{1}{4} \sum_{i=1}^{n} f_{i}^{2}\right) d s+\frac{1}{4} \int_{0}^{1} \frac{d \sigma_{2}}{d s}\left[\frac{\left|\dot{\sigma}_{1}\right|}{\frac{d \sigma_{2}}{d s}}+\sqrt{2} \alpha\right]^{2} d s\right\}
\end{aligned}
$$

If $\Psi(x, 0)<0$, then

$$
\frac{u(x, t)}{u\left(x_{0}, t_{0}\right)} \geq\left(\frac{t}{t_{0}}\right)^{-\frac{n}{2}} \exp \left(-\int_{t_{0}}^{t} 2 \sqrt{n} c(\tau) d \tau\right) \exp \left[-d\left(\left(x_{0}, t_{0}\right),(x, t)\right)\right]
$$

Proof. The proof is same as the proof of Proposition 3.1.

4. $L^{1}$ and Pointwise Estimate. Let us first recall some general theory of parabolic equations for the sake of convenience to the readers.

Theorem 4.1. (c.f. P.43 of [Fr]) Consider the operator

$$
L u:=\sum_{i, j=1}^{n} a_{i j}(x, t) \frac{\partial^{2} u}{\partial x_{i} \partial x_{j}}+\sum_{i=1}^{n} b_{i}(x, t) \frac{\partial u}{\partial x_{i}}+c(x, t) u-\frac{\partial u}{\partial t}
$$

on $\mathbb{R}^{n} \times[0, T]$. Assume that $L$ is parabolic in $\mathbb{R}^{n} \times[0, T]$, i.e. for every $(x, t) \in$ $\mathbb{R}^{n} \times[0, T]$ and for every real vector $\zeta \neq 0, \sum a_{i j}(x, t) \zeta_{i} \zeta_{j}>0$. Assume also that the coefficients of $L$ are continous functions in $\mathbb{R}^{n} \times[0, T]$ with the following growth conditions

$$
\begin{aligned}
& \left|a_{i j}(x, t)\right| \leq M,\left|b_{i}(x, t)\right| \leq M(1+|x|), c(x, t) \leq M\left(1+|x|^{2}\right) \\
& \text { for } i, j,=1, \cdots, n,(x, t) \in \mathbb{R}^{n} \times(0, T], M=\text { positive constant } .
\end{aligned}
$$

Assume further that $L u \leq 0$ in $\mathbb{R}^{n} \times(0, T]$ and that

$$
u(x, t) \geq-B \exp \left[\beta|x|^{2}\right] \quad \text { in } \quad \mathbb{R}^{n} \times[0, T]
$$


for some positive constants $B, \beta$. If $u(x, 0) \geq 0$ in $\mathbb{R}^{n}$, then $u(x, t) \geq 0$ in $\mathbb{R}^{n} \times[0, T]$.

Theorem 4.2. (c.f. P. 44[Fr]) Consider the parabolic operator

$$
L u:=\sum_{i, j=1}^{n} a_{i j}(x, t) \frac{\partial^{2} u}{\partial x_{i} \partial x_{j}}+\sum_{i=1}^{n} b_{i}(x, t) \frac{\partial x}{\partial x_{i}}+c(x, t) u-\frac{\partial u}{\partial t}
$$

with continous coefficients in $\mathbb{R}^{n} \times(0, T]$. Let (4.1) be satisfied. then there exists at most one solution to the Cauchy problem

$$
\left\{\begin{array}{lll}
L u=f(x, t) & \text { in } & \mathbb{R}^{n} \times(0, T] \\
u(x, t)=\varphi(x) & \text { in } & \mathbb{R}^{n}
\end{array}\right.
$$

satisfying

$$
|u(x, t)| \leq B \exp \left[\beta|x|^{2}\right]
$$

for some positive constant $B, \beta$.

Let $u_{R}>0$ be a positive solution of the equation

$$
\frac{\partial u}{\partial t}=\Delta u+\sum_{i=1}^{n} f_{i} \frac{\partial u}{\partial x_{i}}-V u
$$

on the ball $B_{R}=\left\{x \in \mathbb{R}^{n}: \theta(x)=|x|-R \leq 0\right\}$ with the Neumann condition $\frac{\partial u_{R}}{\partial \nu}=$ 0 on $\partial B_{R}$ where $\nu=\nabla \theta$. Assume that (2.31)-(2.41) hold. Then for $\varphi=-\log u_{R}$, we have

$$
\varphi_{t}+|\nabla \varphi|^{2}-\sum_{j=1}^{n} f_{j} \frac{\partial \varphi}{\partial x_{j}}-V<\alpha \sqrt{|\nabla \varphi|+\beta}+\frac{n}{2 t}+2 \sqrt{n} c(t)
$$

on $B_{R}$ as long as it holds for $t=0$.

From now on, we shall study the behaviour of the solution of equation (4.5) in $\mathbb{R}^{n}$. If the initial data is nonnegative, then in view of Theorem 4.1 the solution is nonnegative. Hence we can assume that the solution of (4.5) is nonnegative.

THEOREM 4.3. Let $u$ be a nonnegative solution of the equatoin

$$
\frac{\partial u}{\partial t}=\Delta u+\sum_{i=1}^{n} f_{i} \frac{\partial u}{\partial x_{i}}-V u
$$

on $\mathbb{R}^{n}$. Suppose that $u(x, 0)$ has compact support ${ }^{*}$,

$$
|f(x, t)| \leq c(t)(1+|x|),|\nabla f(x, t)|=\sqrt{\sum_{i=1}^{n}\left|\nabla f_{i}\right|^{2}} \leq c(t)(1+|x|)
$$

and

$$
|V(x, t)| \leq c(t)\left(1+|x|^{2}\right)
$$

*It is sufficient for $u(x, 0)$ to decay like Gaussian. 
Let $a_{1}(t), a_{2}(t)$ and $a_{3}(t)$ satisfy the following ordinary differential equations

$$
\begin{aligned}
& \left(\log a_{1}\right)^{\prime}+2 n a_{2}+a_{3}^{2}+4 a_{2} c+c=0 \\
& a_{2}^{\prime}+4 a_{2}^{2}+4 a_{2} c+c=0 \\
& a_{3}^{\prime}+2 a_{3} c-\sqrt{2 n} c=0
\end{aligned}
$$

with initial conditions suitably chosen (e.g. $\left.a_{1}(0)>0, a_{2}(0)>\frac{1}{2} a_{3}(0)>0\right)$ so that $a_{1}(t)>0, a_{2}(t) \geq \frac{1}{2} a_{3}(t)>0$ for all $0 \leq t \leq T$ for some $T>0$. Then, for $0 \leq t \leq T$,

$$
\begin{aligned}
& a_{1}(t) \int_{\mathbb{R}^{n}} u(x, t) \exp \left(a_{2}(t)|x|^{2}-a_{3}(t) \sqrt{1+|x|^{2}}\right) \\
\leq & a_{1}(0) \int_{\mathbb{R}^{n}} u(x, 0) \exp \left(a_{2}(0)|x|^{2}-a_{3}(0) \sqrt{1+|x|^{2}}\right)
\end{aligned}
$$

Proof. Let $\rho$ be any smooth function and $B_{R}=\left\{x \in \mathbb{R}^{n}:|x| \leq R\right\}$. We have

$$
\begin{aligned}
& \frac{d}{d t} \int_{B_{R}} \rho u=\int_{B_{R}} \rho_{t} u+\int_{B_{R}} \rho u_{t} \\
= & \int_{B_{R}} \rho_{t} u+\int_{B_{R}} \rho \Delta u+\int_{B_{R}} \rho \sum_{i=1}^{n} f_{i} \frac{\partial u}{\partial x_{i}}-\int_{B_{R}} \rho V u \\
= & \int_{B_{R}} \rho_{t} u+\int_{B_{R}}(\Delta \rho) u-\int_{\partial B_{R}} u \frac{\partial \rho}{\partial \nu} d s+\int_{\partial B_{R}} \rho \frac{\partial u}{\partial \nu} d s \\
- & \int_{B_{R}}\left(\rho \sum_{i=1}^{n} f_{i, i}+\sum_{i=1}^{n} \rho_{i} f_{i}\right) u+\int_{\partial B_{R}} u \rho f \cdot \nu d s-\int_{B_{R}} \rho V u
\end{aligned}
$$

by Divergence Theorem and Green's first identity. Now we can use simple cut off argument to otbain the following

$$
\frac{d}{d t} \int_{\mathbb{R}^{n}} \rho u=\int_{\mathbb{R}^{n}}\left(\rho_{t}+\Delta \rho-\sum_{i=1}^{n} f_{i} \rho_{i}-\sum_{i=1}^{n} f_{i, i} \rho-V \rho\right) u
$$

Observe that

$$
\begin{aligned}
\left|\sum_{i=1}^{n} f_{i, i}+V\right| & \leq \sum_{i=1}^{n}\left|f_{i, i}\right|+|V| \\
& \leq \sqrt{n} \sqrt{\sum_{i=1}^{n}\left|f_{i, i}\right|^{2}}+c\left(1+|x|^{2}\right) \\
& \leq \sqrt{n} c(1+|x|)+c\left(1+|x|^{2}\right) \\
& \leq \sqrt{2 n} c\left(1+|x|^{2}\right)^{\frac{1}{2}}+c\left(1+|x|^{2}\right) \\
\left|\sum_{i=1}^{n} x_{i} f_{i}\right| & \leq|x||f| \leq c|x|(1+|x|) \\
& \leq c(1+|x|)^{2} \leq 2 c\left(1+|x|^{2}\right)
\end{aligned}
$$

Set

$$
\rho=a_{1}(t) \exp \left[a_{2}(t)|x|^{2}-a_{3}(t) \sqrt{1+|x|^{2}}\right]
$$


Then

$$
\begin{aligned}
\rho_{t} & =\left(a_{t}^{\prime}+a_{1}(t) a_{2}^{\prime}(t)|x|^{2}-a_{1}(t) a_{3}^{\prime}(t) \sqrt{1+|x|^{2}}\right) \exp \left[a_{2}(t)|x|^{2}-a_{3}(t) \sqrt{1+|x|^{2}}\right] \\
& =\left(\left(\log a_{1}\right)^{\prime}+a_{2}^{\prime}|x|^{2}-a_{3}^{\prime} \sqrt{1+|x|^{2}}\right) \rho \\
\rho_{i} & =\left(2 x_{i} a_{2}(t)-a_{3}(t) \frac{x_{i}}{\sqrt{1+|x|^{2}}}\right) \rho \\
\rho_{i i} & =\left(2 a_{2}(t)-\frac{a_{3}(t)}{\sqrt{1+|x|^{2}}}+\frac{a_{3}(t) x_{i}^{2}}{\left(1+|x|^{2}\right)^{3 / 2}}\right) \rho \\
& +\left(2 x_{i} a_{2}(t)-a_{3}(t) \frac{x_{i}}{\sqrt{1+|x|^{2}}}\right)^{2} \rho \\
& =\left[2 a_{2}(t)-\frac{a_{3}(t)}{\sqrt{1+|x|^{2}}}+\frac{a_{3}(t) x_{i}^{2}}{\left(1+|x|^{2}\right)^{3 / 2}}+\left(2 a_{2}(t)-\frac{a_{3}(t)}{\sqrt{1+|x|^{2}}}\right)^{2} x_{i}^{2}\right] \rho \\
\triangle \rho & =\left[2 n a_{2}(t)-\frac{n a_{3}(t)}{\sqrt{1+|x|^{2}}}+\frac{a_{3}(t)|x|^{2}}{\left(1+|x|^{2}\right)^{3 / 2}}+\left(2 a_{2}(t)-\frac{a_{3}(t)}{\sqrt{1+|x|^{2}}}\right)^{2}|x|^{2}\right] \rho
\end{aligned}
$$

It follows that

$$
\begin{aligned}
& \rho_{t}+\Delta \rho-\sum_{i=1}^{n} f_{i} \rho_{i}-\left(\sum_{i=1}^{n} f_{i, i}+V\right) \rho \\
= & {\left[\left(\log a_{1}\right)^{\prime}+a_{2}^{\prime}|x|^{2}-a_{3}^{\prime} \sqrt{1+|x|^{2}}+2 n a_{2}-\frac{\left((n-1)|x|^{2}+n\right) a_{3}}{\left(1+|x|^{2}\right)^{3 / 2}}\right.} \\
& \left.+\left(2 a_{2}-\frac{a_{3}}{\sqrt{1+|x|^{2}}}\right)^{2}|x|^{2}-\left(2 a_{2}-\frac{a_{3}}{\sqrt{1+|x|^{2}}}\right) \sum_{i=1}^{n} x_{i} f_{i}-\left(\sum_{i=1}^{n} f_{i, i}+V\right)\right] \rho
\end{aligned}
$$

Recall that

$$
2 a_{2}(t) \geq a_{3}(t) \geq \frac{a_{3}(t)}{\sqrt{1+|x|^{2}}} \quad 0 \leq t \leq T
$$

Therefore (4.11) has the following upper estimates

$$
\begin{aligned}
& \rho_{t}+\Delta \rho-\sum_{i=1}^{n} f_{i} \rho_{i}-\left(\sum_{i=1}^{n} f_{i, i}+V\right) \rho \\
\leq & {\left[\left(\log a_{1}\right)^{\prime}+a_{2}^{\prime}|x|^{2}-a_{3}^{\prime} \sqrt{1+|x|^{2}}+2 n a_{2}-\frac{\left((n-1)|x|^{2}+n\right) a_{3}}{\left(1+|x|^{2}\right)^{3 / 2}}\right.} \\
& +\left(4 a_{2}^{2}-\frac{4 a_{2} a_{3}}{\sqrt{1+|x|^{2}}}+\frac{a_{3}^{2}}{1+|x|^{2}}\right)|x|^{2}+\left(2 a_{2}-\frac{a_{3}}{\sqrt{1+|x|^{2}}}\right) 2 c\left(1+|x|^{2}\right) \\
& \left.+\sqrt{2 n} c\left(1+|x|^{2}\right)^{\frac{1}{2}}+c\left(1+|x|^{2}\right)\right] \rho \\
= & {\left[\left(\log a_{1}\right)^{\prime}+a_{2}^{\prime}|x|^{2}-a_{3}^{\prime} \sqrt{1+|x|^{2}}+2 n a_{2}-\frac{(n-1) a_{3}}{\left(1+|x|^{2}\right)^{1 / 2}}\right.} \\
& -\frac{a_{3}}{\left(1+|x|^{2}\right)^{3 / 2}}+4 a_{2}^{2}|x|^{2}-\frac{4 a_{2} a_{3}|x|^{2}}{\sqrt{1+|x|^{2}}}+a_{3}^{2}-\frac{a_{3}^{2}}{1+|x|^{2}} \\
& +4 a_{2} c+4 a_{2} c|x|^{2}-2 a_{3} c \sqrt{1+|x|^{2}}+\sqrt{2 n} c\left(1+|x|^{2}\right)^{\frac{1}{2}} \\
& \left.+c+c|x|^{2}\right] \rho
\end{aligned}
$$




$$
\begin{aligned}
= & {\left[\left(\log a_{1}\right)^{\prime}+2 n a_{2}+a_{3}^{2}+4 a_{2} c+c+\left(a_{2}^{\prime}+4 a_{2}^{2}+4 a_{2} c+c\right)|x|^{2}\right.} \\
& +\left(-a_{3}^{\prime}-2 a_{3} c+\sqrt{2 n} c\right)\left(1+|x|^{2}\right)^{\frac{1}{2}}-\frac{(n-1) a_{3}}{\left(1+|x|^{2}\right)^{\frac{1}{2}}} \\
& \left.-\frac{a_{3}}{\left(1+|x|^{2}\right)^{3 / 2}}-\frac{4 a_{2} a_{3}|x|^{2}}{\sqrt{1+|x|^{2}}}-\frac{a_{3}^{2}}{1+|x|^{2}}\right] \rho \\
\leq & {\left[\left(\log a_{1}\right)^{\prime}+2 n a_{2}+a_{3}^{2}+4 a_{2} c+c+\left(a_{2}^{\prime}+4 a_{2}^{2}+4 a_{2} c+c\right)|x|^{2}\right.} \\
& \left.+\left(-a_{3}^{\prime}-2 a_{3} c+\sqrt{2 n} c\right)\left(1+|x|^{2}\right)^{\frac{1}{2}}\right] \rho \\
\leq & 0
\end{aligned}
$$

because of (4.6), (4.7) and (4.8) and the assumption $a_{2}(t) \geq \frac{1}{2} a_{3}(t)>0$ for all $0 \leq t \leq T$ (4.10) and (4.12) imply

$$
\frac{d}{d t} \int_{\mathbb{R}^{n}} \rho u \leq 0
$$

(4.9) follows immediately from the above inequality.

Theorem 4.3 above gives $L^{1}$-estimate for $u$. Combining the Harnack inequality of $\S 3$, we shall have the following pointwise estimate of $u$.

THEOREM 4.4. Let $u$ be a nonnegative solution of the equation

$$
\frac{\partial u}{\partial t}=\Delta u+\sum_{i=1}^{n} f_{i} \frac{\partial u}{\partial x_{i}}-V u
$$

on $\mathbb{R}^{n}$. Suppose that $u(x, 0)$ has compact support and $^{\dagger}|f(x, t)| \leq c(t)(1+|x|)$, $|\nabla f(x, t)|=\sqrt{\sum_{i=1}^{n}\left|\nabla f_{i}\right|^{2}} \leq c(t)(1+|x|),|V(x, t)| \leq c(t)\left(1+|x|^{2}\right)$. Let $a_{1}(t), a_{2}(t)$ and $a_{3}(t)$ satisfy the ordinary differential equations (4.6), (4.7) and (4.8) with initial conditions suitably chosen (e.g. $\left.a_{1}(0)>0, a_{2}(0)>\frac{1}{2} a_{3}(0)>0\right)$ so that $a_{1}(t)>$ $0, a_{2}(t) \geq \frac{1}{2} a_{3}(t)>0$ for all $0 \leq t \leq 2 T$ for some $T>0$. Assume that the Harnack inequality holds

$$
\frac{u(x, t)}{u\left(x_{0}, t_{0}\right)} \geq\left(\frac{t}{t_{0}}\right)^{-\frac{n}{2}}\left[\exp \left(-\int_{t_{0}}^{t} 2 \sqrt{n} c(\tau) d \tau\right)\right] \exp \left[-d\left(\left(x_{0}, t_{0}\right),(x, t)\right)\right]
$$

where

$$
\begin{aligned}
d\left(\left(x_{0}, t_{0}\right),(x, t)\right):=\inf _{\sigma \in \mathcal{P}} & \left\{\frac{1}{2} \int_{0}^{1}<\dot{\sigma_{1}}, f>d s+\int_{0}^{1} \frac{d \sigma_{2}}{d s}\left(\sqrt{2} \alpha \sqrt{\frac{|f|^{2}}{4}+\frac{\beta}{2}}+V\right.\right. \\
& \left.\left.+\frac{1}{4} \sum_{i=1}^{n} f_{i}^{2}\right) d s+\frac{1}{4} \int_{0}^{1} \frac{d \sigma_{2}}{d s}\left[\frac{\left|\sigma_{1}\right|}{\frac{d \sigma_{2}}{d s}}+\sqrt{2} \alpha\right]^{2} d s\right\}
\end{aligned}
$$

\footnotetext{
†t is sufficient for $u(x, 0)$ to decay like Gaussian
} 
Here $\mathcal{P}=\left\{\right.$ differentiable path $\sigma=\left(\sigma_{1}, \sigma_{2}\right):[0,1] \rightarrow \mathbb{R}^{n} \times \mathbb{R}$ such that $\sigma(0)=$ $\left.\left(\sigma_{1}(0), \sigma_{2}(0)\right)=\left(x_{0}, t_{0}\right), \sigma(1)=(x, t), \sigma_{2}^{\prime}(s)>0\right\}$ and $\alpha, \beta$ are defined in Theorem 2.3. Then for $t \leq T$,

$$
\begin{aligned}
u(x, t) \leq & 2^{\frac{n}{2}} \frac{a_{1}(0)}{a_{1}(2 t)}\left(\exp \int_{t}^{2 t} 2 \sqrt{n} c(\tau) d \tau\right) \\
& {\left[\int_{x \in \mathbb{R}^{n}} u(x, 0) \exp \left(a_{2}(0)|x|^{2}-a_{3}(0) \sqrt{1+|x|^{2}}\right)\right] } \\
& \left\{\int_{y \in \mathbb{R}^{n}}[\exp (-d(x, t),(y, 2 t))] \exp \left[a_{2}(2 t)|y|^{2}-a_{3}(2 t) \sqrt{1+|y|^{2}}\right]\right\}^{-1}
\end{aligned}
$$

Proof. By the $L^{1}$-estimate for $u$ and Harnack inequality, we have

$$
\begin{aligned}
& a_{1}(0) \int_{x \in \mathbb{R}^{n}} u(x, 0) \exp \left[a_{2}(0)|x|^{2}-a_{3}(0) \sqrt{1+|x|^{2}}\right] \\
\geq & a_{1}(2 t) \int_{y \in \mathbb{R}^{n}} u(y, 2 t) \exp \left[a_{2}(2 t)|y|^{2}-a_{3}(2 t) \sqrt{1+|y|^{2}}\right] \\
\geq & a_{1}(2 t) \int_{y \in \mathbb{R}^{n}} u(x, t)\left(\frac{2 t}{t}\right)^{-\frac{n}{2}}\left[\exp \left(-\int_{t}^{2 t} 2 \sqrt{n} c(\tau) d \tau\right)\right] \exp [(-d((x, t),(y, 2 t))] \\
& \quad\left[\exp \left(a_{2}(2 t)|y|^{2}-a_{3}(2 t) \sqrt{1+|y|^{2}}\right)\right] \\
= & \left(\frac{1}{2}\right)^{\frac{n}{2}} a_{1}(2 t) u(x, t)\left[\exp \left(-\int_{t}^{2 t} 2 \sqrt{n} c(\tau) d \tau\right)\right] \int_{y \in \mathbb{R}^{n}}[\exp (-d(x, t),(y, 2 t))] \\
& \left.\quad \exp \left(a_{2}(2 t)|y|^{2}-a_{3}(2 t) \sqrt{1+|y|^{2}}\right)\right]
\end{aligned}
$$

The conclusion follows immediately.

Corollary 4.1. Under the assumptions of Theorem 2.4, Theorem 4.4, $u(x, t)$ decays like a Gaussian.

Proof. In view of Theorem 4.4, we need the following lower estimate of

$$
\begin{aligned}
& \int_{y \in \mathbb{R}^{n}}[\exp -d(((x, t),(y, 2 t)))]\left[\exp \left(a_{2}(2 t)|y|^{2}-a_{3}(2 t) \sqrt{1+|y|^{2}}\right)\right] \\
\geq & \int_{y \in \mathbb{R}^{n}}\left[\operatorname { e x p } \left\{-\frac{1}{2} \int_{0}^{1}<y-x, f((1-s) x+s y,(1-s) t+2 s t)>d s\right.\right. \\
& +t \int_{0}^{1}\{\sqrt{2} \alpha((1-s) t+2 s t) \\
& \left.+\frac{1}{4} \sum_{i=1}^{n} f_{i}^{2}((1-s) x+s y,(1-s) t+2 s t)+V((1-s) x+s y,(1-s) t+2 s t)\right\} d s \\
+ & \left.\frac{|y-x|^{2}}{4 t}+\frac{\sqrt{2}}{2}|y-x| \int_{0}^{2} \alpha((1-s) x+s y,(1-s) t+2 s t)+\frac{1}{2} \beta((1-s) x+s y,(1-s)+2 s t)\right]^{\frac{1}{2}} \\
& \exp \left(a_{2}(2 t)|y|^{2}-a_{3}(2 t) \sqrt{1+|y|^{2}}\right)
\end{aligned}
$$


Observe that in view of the Remark after Theorem 2.4, we have

$$
\begin{aligned}
& {\left[\frac{|f((1-s) x+s y,(1+s) t)|^{2}}{4}+\frac{\beta((1-s) x+s y,(1+s) t)}{2}\right]^{\frac{1}{2}}} \\
& \quad \leq \frac{|f((1-s) x+s y,(1-s) t)|}{2}+\frac{1}{\sqrt{2}} \sqrt{\beta((1-s) x+s y,(1+s) t)} \\
& \quad \leq \frac{c}{2}(1+(1-s)|x|+s|y|)+\frac{1}{\sqrt{2}} \sqrt{c^{4 / 3}|(1-s) x+s y|^{2}+\frac{4}{(T-t)^{2}}+\frac{n}{2 t}} \\
& \quad \leq \frac{c}{2}(1+(1-s)|x|+s|y|)+\frac{c^{2 / 3}}{\sqrt{2}}((1-s)|x|+s|y|)+\frac{1}{\sqrt{2}}\left(\frac{4}{(T-t)^{2}}+\frac{n}{2 t}\right)^{\frac{1}{2}} \\
& \quad \leq\left(\frac{c}{2}+\frac{c^{2 / 3}}{\sqrt{2}}\right)(1-s)|x|+\left(\frac{c}{2}+\frac{c^{2 / 3}}{\sqrt{2}}\right) s|y|+\frac{c}{2}+\frac{2}{T-t}+\frac{\sqrt{n}}{\sqrt{2 t}}
\end{aligned}
$$

Hence

$$
\begin{gathered}
\sqrt{2} \alpha((1-s) t+2 s t)\left[\frac{|f((1-s) x+s y,(1+s) t)|^{2}}{4}\right. \\
\left.+\frac{\beta((1-s) x+s y,(1-s) t+2 s t)}{2}\right]^{\frac{1}{2}} \\
\geq-\frac{\sqrt{2}}{T-(1+s) t}\left[\left(\frac{c}{2}+\frac{c^{2 / 3}}{\sqrt{2}}\right)(1-s)|x|+\left(\frac{c}{2}+\frac{c^{2 / 3}}{\sqrt{2}}\right) s|y|+\frac{c}{2}+\frac{2}{T-t}+\frac{\sqrt{n}}{\sqrt{2} t}\right]
\end{gathered}
$$

On the other hand, we also have the following estimates

$$
\begin{aligned}
V((1-s) x+s y,(1-s) t+2 s t) & \leq c\left(1+|(1-s) x+s y|^{2}\right) \\
& \leq c\left(1+2(1-s)^{2}|x|^{2}+2 s^{2}|y|^{2}\right) \\
\frac{1}{4}|f((1-s) x+s y,(1-s) t+2 s t)|^{2} & \leq \frac{1}{4} c^{2}(1+|(1-s) x+s y|)^{2} \\
& \leq \frac{c^{2}}{2}\left(1+|(1-s) x+s y|^{2}\right) \\
& \leq \frac{c^{2}}{2}\left(1+2(1-s)^{2}|x|^{2}+2 s^{2}|y|^{2}\right) \\
<y-x, f((1-s) x+s y & (1-s) t+2 s t)> \\
& \leq|y-x||f((1-s) x+s y,(1+s) t)| \\
& \leq(|x|+|y|) c(1+(1-s)|x|+s|y|) \\
& =c\left((1-s)|x|^{2}+|x||y|+|x|+s|y|^{2}+|y|\right)
\end{aligned}
$$

Therefore

$$
\begin{aligned}
\int_{y \in \mathbb{R}^{n}} & \{\exp [-d((x, t),(y, 2 t))]\} \exp \left(a_{2}(2 t)|y|^{2}-a_{3}(2 t) \sqrt{1+|y|^{2}}\right) \\
\geq \int_{y \in \mathbb{R}^{n}} & {\left[\operatorname { e x p } \left\{-\frac{c}{2} \int_{0}^{1}\left[(1-s)|x|^{2}+|x||y|+|x|+s|y|^{2}+|y|\right] d s\right.\right.} \\
-t & \int_{0}^{1}\left\{\frac{\sqrt{2}}{T-(1+s) t}\left[\frac{c}{2}+\frac{c^{2 / 3}}{\sqrt{2}}\right)((1-s)|x|+s|y|)+\frac{c}{2}+\frac{2}{T-t}+\frac{\sqrt{n}}{\sqrt{2} t}\right] \\
& \left.+\frac{c^{2}}{2}\left(1+2(1-s)^{2}|x|^{2}+2 s^{2}+|y|^{2}\right)+c\left(1+2(1-s)^{2}|x|^{2}+2 s^{2}|y|^{2}\right)\right\} d s
\end{aligned}
$$




$$
\begin{gathered}
\left.\left.+\frac{|x-y|^{2}}{4 t}+\frac{\sqrt{2}}{2}|x-y| \int_{0}^{1} \frac{d s}{T-(1+s) t}+\frac{t}{2} \int_{0}^{1} \frac{d s}{[T-(1+s) t]^{2}}\right\}\right] \\
\exp \left(a_{2}(2 t)|y|^{2}-a_{3}(2 t) \sqrt{1+|y|^{2}}\right) \\
=\int_{y \in \mathbb{R}^{n}} \exp \left\{\left[-\frac{c}{4}-\frac{t}{3}\left(c^{2}+2 c\right)+\frac{1}{4 t}\right]|x|^{2}+\text { lower order term in }|x|\right\} \\
\exp \left(a_{2}(2 t)|y|^{2}-a_{3}(2 t) \sqrt{1+|y|^{2}}\right)
\end{gathered}
$$

Observe that when $t$ is sufficiently small, $\frac{1}{4 t}-\frac{t}{2}\left(c^{2}+2 c\right)-\frac{c}{4}$ is very positive. Therefore $u(x, t)$ decays like a Gaussian.

5. The Duncan-Mortensen-Zakai equation. In the nonlinear filtering, we have the following signal observation model:

$$
\left\{\begin{array}{lll}
d x(t)=f(x(t)) d t+g(x(t)) d v(t) & , & x(0)=x_{0} \\
d y(t)=h(x(t)) d t+d w(t) & , & y(0)=0
\end{array}\right.
$$

in which $x, v, y$ and $w$ are vector valued processes and $v$ and $w$ have components which are independent, standard Brownian processes.

Let $\rho$ denote the conditional probability density of the state given the observation $\{y(t): 0 \leq s \leq t\}$. Then $\rho$ can be obtained by normalizing $\sigma$ which satisfies the Duncan-Mortensen-Zakai equation.

$$
d \sigma=L_{0}(\sigma) d t+\sum_{i=1}^{m} L_{i}(\sigma) d y_{i}, \sigma(0, x)=\sigma_{0}
$$

where $L_{0}=\frac{1}{2} \Delta-\sum_{i=1}^{n} f_{i} \frac{\partial}{\partial x_{i}}-\sum_{n=1}^{n} \frac{\partial f_{i}}{\partial x_{i}}-\frac{1}{2} \sum_{i=1}^{m} h_{i}^{2}$ and $L_{i}$ is the multiplication operator by $h_{i}$.

While (5.2) is a stochastic differential equation, Davis reduces it to a time varying partial differential equation by introducing a new unnormalized density $u=$ $\exp \left(\frac{1}{2} \sum_{i=1}^{m} h_{i}^{2}(x) y_{i}(t)\right) \sigma$, which satisfies the following equation

$$
\frac{\partial u}{\partial t}=L_{0} u+\sum_{i=1}^{m} y_{i}(t)\left[L_{0}, L_{i}\right] u+\frac{1}{2} \sum_{i, j=1}^{n} y_{i}(t) y_{j}(t)\left[\left[L_{0}, L_{i}\right], L_{j}\right] u
$$

We can rewrite this equation as

$$
\begin{aligned}
\frac{\partial u}{\partial t} & =\frac{1}{2} \Delta u+\sum_{i=1}^{n}\left(-f_{i}(x)+\sum_{j=1}^{m} y_{j} \frac{\partial h_{j}}{\partial x_{i}}\right) \frac{\partial u}{\partial x_{i}}-\left(\sum_{i=1}^{n} f_{i, i}+\frac{1}{2} \sum_{i=1}^{M} h_{i}^{2}\right. \\
& \left.-\frac{1}{2} \sum_{i=1}^{m} y_{i} \Delta h_{i}+\sum_{i=1}^{m} \sum_{j=1}^{n} y_{i} f_{j} \frac{\partial h_{i}}{\partial x_{j}}-\frac{1}{2} \sum_{i=1}^{m} \sum_{j=1}^{m} \sum_{k=1}^{n} y_{i} y_{j} \frac{\partial h_{i}}{\partial x_{k}} \frac{\partial h_{j}}{\partial x_{k}}\right) u
\end{aligned}
$$

By changing variables from $x_{i}$ to $\sqrt{2} x_{i}$ and by letting

$$
\bar{u}(x, t)=u\left(\frac{x}{\sqrt{2}}, t\right)
$$




$$
\begin{aligned}
& \bar{f}_{i}(x)=f_{i}\left(\frac{x}{\sqrt{2}}\right) \\
& \bar{h}_{i}(x)=h_{i}\left(\frac{x}{\sqrt{2}}\right)
\end{aligned}
$$

we obtain

$$
\begin{aligned}
\frac{\partial \bar{u}}{\partial t} & =\Delta \bar{u}+\sum_{i=1}^{n}\left(-\sqrt{2} \bar{f}_{i}+2 \sum_{j=1}^{m} y_{j} \frac{\partial \bar{h}_{j}}{\partial x_{i}}\right) \frac{\partial \bar{u}}{\partial x_{i}}-\left(\sqrt{2} \sum_{i=1}^{n} \bar{f}_{i, i}+\frac{1}{2} \sum_{i=1}^{m} \bar{h}_{i}^{2}\right. \\
& \left.-\sum_{i=1}^{m} y_{i} \Delta \bar{h}_{i}+\sqrt{2} \sum_{i=1}^{m} \sum_{j=1}^{n} y_{i} \bar{f}_{j} \frac{\partial \bar{h}_{i}}{\partial x_{j}}-\sum_{i=1}^{m} \sum_{j=1}^{m} \sum_{k=1}^{n} y_{i} y_{i} \frac{\partial \bar{h}_{i}}{\partial x_{k}} \frac{\partial \bar{h}_{j}}{\partial x_{k}}\right) \bar{u}
\end{aligned}
$$

Hence (5.4) can be rewritten as

$$
\bar{u}_{t}=\Delta \bar{u}+\sum_{i=1}^{n} \tilde{f}_{i} \bar{u}_{i}-\tilde{V} \bar{u}
$$

where

$$
\begin{gathered}
\tilde{f}_{i}=-\sqrt{2} \bar{f}_{i}+2 \sum_{j=1}^{m} y_{j} \frac{\partial \bar{h}_{j}}{\partial x_{i}} \\
\tilde{V}=\sqrt{2} \sum_{i=1}^{n} \bar{f}_{i, i}+\frac{1}{2} \sum_{i=1}^{m} \bar{h}_{i}^{2}-\sum_{i=1}^{m} y_{i} \triangle \bar{h}_{i}+\sqrt{2} \sum_{i=1}^{m} \sum_{j=1}^{n} y_{i} \bar{f}_{j} \frac{\partial \bar{h}_{i}}{\partial x_{j}} \\
-\sum_{i=1}^{m} \sum_{j=1}^{m} \sum_{k=1}^{n} y_{i} y_{j} \frac{\partial \bar{h}_{i}}{\partial x_{k}} \frac{\partial \bar{h}_{j}}{\partial x_{k}}
\end{gathered}
$$

THEOREM 5.1. Let $\bar{u}_{R}>0$ equation (5.5) on the closed ball $B_{R}=\left\{x \in \mathbb{R}^{n}\right.$ : $|x| \leq R\}$ with the Neumann condition $\frac{\partial \bar{u}_{R}}{\partial \nu}=0$ on $\partial B_{R}$. Let $\bar{\varphi}(x, t)=-\log \bar{u}_{R}(x, t)$ and

$$
\bar{\Psi}(x, t)=\bar{\varphi}_{t}+|\nabla \varphi|^{2}-\sum_{j=1}^{n} \tilde{f}_{j} \frac{\partial \bar{\varphi}}{\partial x_{j}}-\tilde{V}-\bar{\alpha}(t) \sqrt{|\nabla \varphi|^{2}+\bar{\beta}(x, t)}-\frac{n}{2 t}-2 \sqrt{n} \bar{c}(t)
$$

Let $\beta(x, t)=\bar{c}_{2}(t)|x|^{2}+4 \bar{\alpha}^{2}(t)+\frac{n}{2 t}$ with $\bar{c}_{2}(t)>0$ and $\bar{\alpha}(t)>0$. Suppose that there exists $\bar{c}_{1}(t)>0$ such that

$$
\begin{aligned}
& |\tilde{f}| \leq \bar{c}(t)(1+|x|) \\
& |\nabla \tilde{f}|=\sqrt{\sum_{i=1}^{n}\left|\nabla \tilde{f}_{i}\right|^{2}} \leq \bar{c}(t),|\nabla \tilde{V}| \leq \bar{c}(1+|x|) \\
& |\triangle \tilde{V}| \leq \bar{c},|\triangle \tilde{f}| \leq \bar{c} \\
& 2 \bar{c}^{2}(t) \leq \bar{c}_{1}(t) \bar{c}_{2}(t) \\
& 2 \bar{c}^{2}(t) \leq \bar{c}_{1}(t)\left(4 \bar{\alpha}^{2}(t)+\frac{n}{2 t}\right)
\end{aligned}
$$




$$
\begin{aligned}
& 4 \bar{c}_{2}(t) \leq \bar{c}_{1}^{2}(t) \\
& \bar{c}_{2}^{\prime}+\frac{3}{t} \bar{c}_{2} \geq 0 \\
& 2\left(\bar{\alpha}^{2}\right)^{\prime}+\frac{6}{t} \bar{\alpha}^{2}+\frac{n}{4 t^{2}}>0 \\
& \bar{\alpha}^{\prime}(t)+\bar{\alpha}\left[\frac{3 \bar{c}}{2 \sqrt{n}}-\bar{\lambda}-\frac{1}{2}\left(\sqrt{\bar{c}_{1}}+\bar{c}_{1}+\bar{c}_{1}^{3 / 2}\right)\right]-\sqrt{n} \bar{c} \geq 0 \\
& \bar{c}^{\prime}(t)+\frac{5}{48 \sqrt{n}} \bar{c}^{2}+\left(\frac{3}{8 t}-\frac{1}{2 \sqrt{n}}\right) \bar{c}-\frac{\sqrt{n}}{2} \bar{c}_{2}>0
\end{aligned}
$$

where $\bar{\lambda}$ is the absolute value of the greatest eigenvalue of $\left(\frac{\tilde{f}_{i, j}+\tilde{f}_{j, i}}{2}\right)$.

$$
\frac{\partial \tilde{V}}{\partial \nu}>\frac{R}{6}\left(1+\frac{\sqrt{n-1}}{R}\right)^{2}+\frac{\bar{\alpha}(t)}{2} \bar{c}_{1}(t) \text { on } \partial B_{R} \text { for } t>0 .
$$

If $\bar{\Psi}(x, 0)<0$, then $\bar{\Psi}(x, t)<0$ for all $t>0$, i.e.,

$$
\begin{gathered}
\bar{\varphi}_{t}+|\nabla \bar{\varphi}|^{2}-\sum_{j=1}^{n} \tilde{f}_{j} \frac{\partial \bar{\varphi}}{\partial x_{j}}-\tilde{V} \\
<\bar{\alpha}(t) \sqrt{|\nabla \bar{\varphi}|^{2}+\bar{\beta}(x, t)}+\frac{n}{2 t}+2 \sqrt{n} \bar{c}(t) \text { for all } t \geq 0
\end{gathered}
$$

Proposition 5.2. Assume

$$
\begin{aligned}
|f| & \leq c(1+|x|),|\nabla f|=\sqrt{\sum_{i=1}^{n}\left|\nabla f_{i}\right|^{2}} \leq c,|H(f)| \\
& =\sqrt{\sum_{j=1}^{n} \sum_{i=1}^{n} \sum_{k=1}^{n}\left(\frac{\partial^{2} f_{j}}{\partial x_{k} \partial x_{i}}\right)^{2}} \leq c \sqrt{\sum_{i=1}^{n}\left|\nabla \Delta f_{i}\right|^{2}} \leq c,|\nabla h| \\
& =\sqrt{\sum_{j=1}^{m}\left|\nabla h_{j}\right|^{2}} \leq c,|H(h)|=\sqrt{\sum_{j=1}^{m} \sum_{i=1}^{n} \sum_{k=1}^{n}\left(\frac{\partial^{2} h_{j}}{\partial x_{k} \partial x_{i}}\right)^{2}} \leq \frac{c}{1+|x|} \\
& \sqrt{\sum_{i=1}^{m}\left|\nabla \Delta h_{i}\right|^{2}} \leq \frac{c}{1+|x|} \text { and } \sqrt{\sum_{i=1}^{m}\left(\Delta \triangle h_{i}\right)^{2}} \leq c
\end{aligned}
$$

Let $\tilde{f}_{i}$ and $\tilde{V}$ be defined as in (5.6) and (5.7). then

(i) $|\tilde{f}| \leq \sqrt{2} c\left(1+\sqrt{\sum_{j=1}^{m} y_{j}^{2}}\right)(1+|x|)$

(ii) $|\nabla \tilde{f}| \leq \sqrt{2} c\left(1+\sqrt{\sum_{j=1}^{m} y_{j}^{2}}\right)$

(iii) $|\tilde{V}| \leq\left(\frac{\sqrt{2 n} c}{2}+c^{2}\right)\left(1+\sqrt{\sum_{j=1}^{m} y_{j}^{2}}\right)^{2}\left(1+|x|^{2}\right)$ 
(iv) $|\nabla \tilde{V}| \leq\left(\frac{\sqrt{n}}{2} c+\frac{n c^{2}}{2}\right)\left(1+\sqrt{\sum_{i=1}^{m} y_{i}^{2}}\right)^{2}(1+|x|)$

(v) $|\triangle \tilde{f}| \leq\left(1+\sqrt{\sum_{j=1}^{m} y_{j}^{2}}\right) c$

(vi) $|\triangle \tilde{V}| \leq\left(\frac{\sqrt{n}}{2} c+c^{2}\right)\left(1+\sqrt{\sum_{j=1}^{m} y_{j}^{2}}\right)^{2}$

\section{Proof.}

(i) $|\tilde{f}|=\left|\sqrt{2} \bar{f}+2 \nabla \sum_{j=1}^{n} y_{j} \bar{h}_{j}\right|$

$$
\begin{aligned}
& \leq \sqrt{2}\left|f\left(\frac{x}{\sqrt{2}}\right)\right|+\sqrt{2}\left|\sum_{j=1}^{n} y_{j}\left(\nabla h_{j}\right)\left(\frac{x}{\sqrt{2}}\right)\right| \\
& \leq \sqrt{2} c\left(1+\frac{|x|}{\sqrt{2}}\right)+\sqrt{2} \sqrt{\sum_{j=1}^{m} y_{j}^{2}} \sqrt{\sum_{j=1}^{n}\left|\nabla h_{j}\right|^{2}\left(\frac{x}{\sqrt{2}}\right)} \\
& \leq \sqrt{2} c(1+|x|)+\sqrt{2} c \sqrt{\sum_{j=1}^{m} y_{j}^{2}} \\
& \leq \sqrt{2} c\left(1+\sqrt{\sum_{j=1}^{m} y_{j}^{2}}\right)(1+|x|)
\end{aligned}
$$

(ii) $|\nabla \tilde{f}|=\sqrt{\sum_{i=1}^{n}\left|\nabla \tilde{f}_{i}\right|^{2}}=\sqrt{\sum_{i=1}^{n}\left|-\sqrt{2} \nabla \tilde{f}_{i}+2 \nabla \sum_{j=1}^{m} y_{j} \frac{\partial \bar{h}_{j}}{\partial x_{i}}\right|^{2}}$

$$
\begin{aligned}
& =\sqrt{\sum_{i=1}^{n}\left|-\left(\nabla f_{i}\right)\left(\frac{x}{\sqrt{2}}\right)+\left[\nabla\left(\sum_{j=1}^{m} y_{i} \frac{\partial h_{j}}{\partial x_{i}}\right)\right]\left(\frac{x}{\sqrt{2}}\right)\right|^{2}} \\
& \leq \sqrt{2} \sqrt{\sum_{i=1}^{n}\left|\left(\nabla f_{i}\right)\left(\frac{x}{\sqrt{2}}\right)\right|^{2}+\sum_{i=1}^{n}\left|\left[\nabla\left(\sum_{j=1}^{m} y_{i} \frac{\partial h_{j}}{\partial x_{i}}\right)\right]\left(\frac{x}{\sqrt{2}}\right)\right|^{2}} \\
& \leq \sqrt{2} \sqrt{\sum_{i=1}^{n}\left|\left(\nabla f_{i}\right)\left(\frac{x}{\sqrt{2}}\right)\right|^{2}}+\sqrt{2} \sqrt{\sum_{i=1}^{n}\left|\left[\nabla\left(\sum_{j=1}^{m} y_{j} \frac{\partial h_{j}}{\partial x_{i}}\right)\right]\left(\frac{x}{\sqrt{2}}\right)\right|^{2}} \\
& \leq \sqrt{2} c+\sqrt{2} \sqrt{\sum_{i=1}^{n} \sum_{k=1}^{n}\left(\sum_{j=1}^{m} y_{j} \frac{\partial^{2} h_{j}}{\partial x_{k} \partial x_{i}}\right)^{2}\left(\frac{x}{\sqrt{2}}\right)} \\
& \leq \sqrt{2} c+\sqrt{2} \sqrt{\sum_{i=1}^{n} \sum_{k=1}^{n}\left(\sum_{j=1}^{m} y_{j}^{2}\right)\left(\sum_{j=1}^{m}\left(\frac{\partial^{2} h_{j}}{\partial x_{k} \partial x_{i}}\right)^{2}\left(\frac{x}{\sqrt{2}}\right)\right)} \\
& \leq \sqrt{2} c+\sqrt{2} \sqrt{\sum_{j=1}^{m} y_{j}^{2}} \sqrt{\sum_{j=1}^{m} \sum_{i=1}^{n} \sum_{k=1}^{n}\left(\frac{\partial^{2} h_{j}}{\partial x_{k} \partial x_{i}}\right)^{2}\left(\frac{x}{\sqrt{2}}\right)}
\end{aligned}
$$




$$
\leq \sqrt{2} c+\sqrt{2} c \sqrt{\sum_{j=1}^{m} y_{j}^{2}}=\sqrt{2} c\left(1+\sqrt{\sum_{j=1}^{m} y_{j}^{2}}\right)
$$

(iii) $|\tilde{V}|=\left|\sqrt{2} \sum_{i=1}^{n} \bar{f}_{i, i}+\frac{1}{2} \sum_{i=1}^{m} \bar{h}_{i}^{2}-\sum_{i=1}^{m} y_{i} \Delta \bar{h}_{i}+\sqrt{2} \sum_{i=1}^{m} \sum_{j=1}^{n} y_{i} \bar{f}_{j} \frac{\partial \bar{h}_{i}}{\partial x_{j}}-\sum_{k=1}^{n}\left(\sum_{i=1}^{m} y_{i} \frac{\partial \bar{h}_{i}}{\partial x_{k}}\right)^{2}\right|$

$$
\begin{aligned}
& =\mid \frac{1}{\sqrt{2}} \sum_{i=1}^{n} f_{i, i}\left(\frac{x}{\sqrt{2}}\right)+\frac{1}{2} \sum_{i=1}^{m} h_{i}^{2}\left(\frac{x}{\sqrt{2}}\right)-\frac{1}{2} \sum_{i=1}^{m} y_{i}\left(\Delta h_{i}\right)\left(\frac{x}{\sqrt{2}}\right) \\
& \quad+\sum_{i=1}^{m} \sum_{j=1}^{n} y_{i} f_{j} \frac{\partial h_{i}}{\partial x_{j}}\left(\frac{x}{\sqrt{2}}\right)-\frac{1}{2} \sum_{k=1}^{n}\left(\sum_{i=1}^{m} y_{i} \frac{\partial h_{i}}{\partial x_{k}}\right)^{2}\left(\frac{x}{\sqrt{2}}\right) \mid \\
& \leq \frac{1}{\sqrt{2}} \sum_{i=1}^{n}\left|\frac{\partial f_{i}}{\partial x_{i}}\right|\left(\frac{x}{\sqrt{2}}\right)+\frac{1}{2} \sum_{i=1}^{m} h_{i}^{2}\left(\frac{x}{\sqrt{2}}\right)+\frac{1}{2} \sqrt{\sum_{i=1}^{m} y_{i}^{2}} \sqrt{\sum_{i=1}^{m}\left(\Delta h_{i}\right)^{2}\left(\frac{x}{\sqrt{2}}\right)}
\end{aligned}
$$$$
+\sum_{i=1}^{m}\left|y_{i}\right| \sqrt{\sum_{j=1}^{n}\left|f_{j}\right|^{2}\left(\frac{x}{\sqrt{2}}\right)} \sqrt{\sum_{j=1}^{n}\left(\frac{\partial h_{i}}{\partial x_{j}}\right)\left(\frac{x}{\sqrt{2}}\right)}
$$$$
+\frac{1}{2} \sum_{k=1}^{n}\left(\sum_{i=1}^{m} y_{i}^{2}\right)\left(\sum_{i=1}^{m}\left(\frac{\partial h_{i}}{\partial x_{k}}\right)^{2}\left(\frac{x}{\sqrt{2}}\right)\right)
$$$$
\leq \sqrt{\frac{n}{2}} \sqrt{\sum_{i=1}^{n}\left(\frac{\partial f_{i}}{\partial x_{i}}\right)^{2}\left(\frac{x}{\sqrt{2}}\right)}+\frac{1}{2} \sum_{i=1}^{m} h_{i}^{2}\left(\frac{x}{\sqrt{2}}\right)+\frac{1}{2} \sqrt{\sum_{i=1}^{m} y_{i}^{2}|\Delta h|\left(\frac{x}{\sqrt{2}}\right)}
$$$$
+\sqrt{\sum_{j=1}^{n} f_{j}^{2}\left(\frac{x}{\sqrt{2}}\right)} \sqrt{\sum_{i=1}^{m} y_{i}^{2}} \sqrt{\sum_{i=1}^{m} \sum_{j=1}^{n}\left(\frac{\partial h_{i}}{\partial x_{j}}\right)^{2}\left(\frac{x}{\sqrt{2}}\right)}+\frac{1}{2}\left(\sum_{i=1}^{m} y_{i}^{2}\right) \sum_{i=1}^{m}\left|\nabla h_{i}\right|^{2}\left(\frac{x}{\sqrt{2}}\right)
$$$$
\leq \sqrt{\frac{n}{2}} c+\frac{c^{2}}{2}(1+|x|)^{2}+\frac{c}{2} \sqrt{\sum_{i=1}^{m} y_{i}^{2}}+c^{2}\left(1+\frac{|x|}{\sqrt{2}}\right) \sqrt{\sum_{i=1}^{m} y_{i}^{2}}+\frac{c^{2}}{2}\left(\sum_{i=1}^{m} y_{i}^{2}\right)
$$$$
\leq \sqrt{\frac{n}{2}} c+\frac{c^{2}}{2}\left(1+|x|^{2}\right)+\frac{c}{2} \sqrt{\sum_{i=1}^{m} y_{i}^{2}}+c^{2}(1+|x|) \sqrt{\sum_{i=1}^{m} y_{i}^{2}}+\frac{c^{2}}{2}\left(\sum_{i=1}^{m} y_{i}^{2}\right)
$$$$
\leq\left[\frac{\sqrt{2 n}}{2} c+c^{2}+\left(\frac{c}{2}+c^{2}\right) \sqrt{\sum_{i=1}^{m} y_{i}^{2}}+\frac{c^{2}}{2} \sum_{i=1}^{m} y_{i}^{2}\right]\left(1+|x|^{2}\right)
$$$$
\leq\left(\frac{\sqrt{2 n} c}{2}+c^{2}\right)\left(1+\sqrt{\sum_{i=1}^{m} y_{i}^{2}}\right)^{2}\left(1+|x|^{2}\right)
$$

$(i v)|\nabla \tilde{V}|=\mid \sqrt{2} \sum_{i=1}^{m} \nabla \bar{f}_{i, i}+\frac{1}{2} \sum_{i=1}^{m} \nabla\left(\bar{h}_{i}^{2}\right)-\sum_{i=1}^{m} y_{i} \nabla\left(\triangle \bar{h}_{i}\right)+\sqrt{2} \sum_{i=1}^{m} \sum_{j=1}^{n} y_{i} \nabla\left(\bar{f}_{j} \frac{\partial \bar{h}_{i}}{\partial x_{j}}\right)$

$$
\begin{gathered}
\sqrt{2} \sum_{i=1}^{m} \nabla \bar{f}_{i, i}+\frac{1}{2} \sum_{i=1}^{m} \nabla\left(\bar{h}_{i}^{2}\right) \\
=\mid \frac{1}{\sqrt{2}} \sum_{i=1}^{n}\left(\nabla \frac{\partial f_{i}}{\partial x_{i}}\right)\left(\frac{x}{\sqrt{2}}\right)+\frac{1}{\sqrt{2}} \sum_{i=1}^{m} h_{i}\left(\frac{x}{\sqrt{2}}\right)\left(\nabla h_{i}\right)\left(\frac{x}{\sqrt{2}}\right)
\end{gathered}
$$




$$
\begin{aligned}
& -\frac{1}{2 \sqrt{2}} \sum_{i=1}^{m} y_{i}\left(\nabla \Delta h_{i}\right)\left(\frac{x}{\sqrt{2}}\right)+\frac{1}{\sqrt{2}} \sum_{i=1}^{m} \sum_{j=1}^{n}\left[\nabla\left(f_{j} \frac{\partial h_{i}}{\partial x_{j}}\right)\right]\left(\frac{x}{\sqrt{2}}\right) \\
& -\frac{1}{2 \sqrt{2}} \sum_{i=1}^{m}\left[\sum_{k=1}^{n} y_{i} \frac{\partial h_{i}}{\partial x_{k}}\left(\frac{x}{\sqrt{2}}\right)\right]\left[\sum_{k=1}^{n} y_{i}\left(\nabla \frac{\partial h_{i}}{\partial x_{k}}\right)\left(\frac{x}{\sqrt{2}}\right)\right] \\
& \leq \frac{1}{\sqrt{2}} \sum_{i=1}^{n}\left|\nabla \frac{\partial f_{i}}{\partial x_{i}}\right|\left(\frac{x}{\sqrt{2}}\right)+\frac{1}{\sqrt{2}} \sum_{i=1}^{m}\left|h_{i}\left(\frac{x}{\sqrt{2}}\right)\right|\left|\nabla h_{i}\right|\left(\frac{x}{\sqrt{2}}\right) \\
& +\frac{1}{2 \sqrt{2}} \sqrt{\sum_{i=1}^{m} y_{i}^{2}} \sqrt{\sum_{i=1}^{m}\left|\nabla \Delta h_{i}\right|^{2}\left(\frac{x}{\sqrt{2}}\right)} \\
& +\frac{1}{\sqrt{2}} \sqrt{\sum_{i=1}^{m} y_{i}^{2}} \sqrt{\sum_{i=1}^{m}\left\{\sum_{i=1}^{n}\left|\nabla h_{j}\right|^{2}\left(\frac{x}{\sqrt{2}}\right)\right\}^{2}} \\
& +\frac{1}{2 \sqrt{2}} \sum_{i=1}^{m}\left[\sqrt{n}\left|y_{i}\right| \sqrt{\sum_{k=1}^{n}\left(\frac{\partial h_{i}}{\partial x_{k}}\right)^{2}\left(\frac{x}{\sqrt{2}}\right)} \sqrt{n}\left|y_{i}\right| \sqrt{\sum_{k=1}^{n}\left|\nabla \frac{\partial h_{i}}{\partial x_{k}}\right|^{2}\left(\frac{x}{\sqrt{2}}\right)}\right. \\
& \leq \frac{\sqrt{n}}{2} \sqrt{\sum_{i=1}^{n}\left|\nabla \frac{\partial f_{i}}{\partial x_{i}}\right|^{2}\left(\frac{x}{\sqrt{2}}\right)}+\frac{1}{\sqrt{2}} \sqrt{\sum_{i=1}^{m} \mid h_{i}\left(\left.\frac{x}{\sqrt{2}}\right|^{2}\right.} \sqrt{\sum_{i=1}^{m}\left|\nabla h_{i}\right|^{2}\left(\frac{x}{\sqrt{2}}\right)} \\
& +\frac{1}{2 \sqrt{2}} \sqrt{\sum_{i=1}^{m} y_{i}^{2}} \sqrt{\sum_{i=1}^{m}\left|\nabla \Delta h_{i}\right|^{2}\left(\frac{x}{\sqrt{2}}\right)} \\
& +\frac{1}{\sqrt{2}} \sqrt{\sum_{i=1}^{m} y_{i}^{2}} \sqrt{\sum_{i=1}^{m}\left\{\sum_{j=1}^{n}\left|\left(\nabla f_{j}\right) \frac{\partial h_{i}}{\partial x_{j}}+f_{j} \nabla \frac{\partial h_{i}}{\partial x_{j}}\right|\left(\frac{x}{\sqrt{2}}\right)\right\}^{2}} \\
& +\frac{n}{2 \sqrt{2}} \sqrt{\sum_{i=1}^{m} y_{i}^{2} \sum_{i=1}^{m} \sum_{k=1}^{n}\left(\frac{\partial h_{i}}{\partial x_{k}}\right)^{2}\left(\frac{x}{\sqrt{2}}\right)} \sqrt{\sum_{i=1}^{m} y_{i}^{2} \sum_{i=1}^{m} \sum_{k=1}^{n}\left|\nabla \frac{\partial h_{i}}{\partial x_{k}}\right|^{2}\left(\frac{x}{\sqrt{2}}\right)} \\
& \leq \frac{\sqrt{n}}{2} \sqrt{\sum_{i=1}^{n} \sum_{k=1}^{n}\left(\frac{\partial^{2} f_{i}}{\partial x_{k} \partial x_{i}}\right)^{2}\left(\frac{x}{\sqrt{2}}\right)}+\frac{1}{\sqrt{2}}\left|h\left(\frac{x}{\sqrt{2}}\right)\right||\nabla h|\left(\frac{x}{\sqrt{2}}\right) \\
& +\frac{1}{2 \sqrt{2}} \sqrt{\sum_{i=1}^{m} y_{i}^{2}} \sqrt{\sum_{i=1}^{m}\left|\nabla\left(\Delta h_{i}\right)\right|^{2}\left(\frac{x}{\sqrt{2}}\right)} \\
& +\frac{1}{\sqrt{2}} \sqrt{\sum_{i=1}^{m} y_{i}^{2}} \sqrt{\sum_{i=1}^{m} n \sum_{j=1}^{n}\left[\left|\nabla f_{j}\right|\left|\frac{\partial h_{i}}{\partial x_{j}}\right|+\left|f_{j}\right|\left|\nabla \frac{\partial h_{i}}{\partial x_{j}}\right|\right]^{2}}\left(\frac{x}{\sqrt{2}}\right) \\
& +\frac{n}{2 \sqrt{2}}\left(\sum_{i=1}^{m} y_{i}^{2}\right) \sqrt{\sum_{i=1}^{m}\left|\nabla h_{i}\right|^{2}\left(\frac{x}{\sqrt{2}}\right)} \sqrt{\sum_{i=1}^{m} \sum_{j=1}^{n} \sum_{k=1}^{n}\left(\frac{\partial^{2} h_{i}}{\partial x_{j} \partial x_{k}}\right)^{2}\left(\frac{x}{\sqrt{2}}\right)} \\
& \leq \frac{\sqrt{n}}{2} c+\frac{1}{\sqrt{2}} c\left(1+\frac{|x|}{\sqrt{2}}\right) c+\frac{1}{2 \sqrt{2}} \sqrt{\sum_{i=1}^{m} y_{i}^{2}} c
\end{aligned}
$$




$$
\begin{aligned}
& +\frac{1}{\sqrt{2}} \sqrt{\sum_{i=1}^{m} y_{i}^{2}} \sqrt{\sum_{i=1}^{m} 2 n \sum_{j=1}^{n}\left[\left|\nabla f_{j}\right|^{2}\left|\frac{\partial h_{i}}{\partial x_{j}}\right|^{2}+\left|f_{j}\right|^{2}\left|\nabla \frac{\partial h_{i}}{\partial x_{j}}\right|^{2}\right]\left(\frac{x}{\sqrt{2}}\right)} \\
& +\frac{n}{2 \sqrt{2}}\left(\sum_{i=1}^{m} y_{i}^{2}\right) c^{2} \\
& \leq \frac{\sqrt{n}}{2} c+\frac{c^{2}}{2}(1+|x|)+\frac{c}{2 \sqrt{2}} \sqrt{\sum_{i=1}^{m} y_{i}^{2}} \\
& +\frac{1}{\sqrt{2}} \sqrt{\sum_{i=1}^{m} y_{i}^{2}} \sqrt{\sum_{i=1}^{m} 2 n \sum_{j=1}^{n}\left|\nabla f_{j}\right|^{2}\left|\frac{\partial h_{i}}{\partial x_{j}}\right|^{2}\left(\frac{x}{\sqrt{2}}\right)} \\
& +\frac{1}{\sqrt{2}} \sqrt{\sum_{i=1}^{m} y_{i}^{2}} \sqrt{\sum_{i=1}^{m} 2 n \sum_{j=1}^{n}\left|f_{j}\right|^{2}\left|\nabla \frac{\partial h_{i}}{\partial x_{j}}\right|^{2}\left(\frac{x}{\sqrt{2}}\right)}+\frac{n}{2 \sqrt{2}}\left(\sum_{i=1}^{m} y_{i}^{2}\right) c^{2} \\
& =\frac{\sqrt{n}}{2} c+\frac{c^{2}}{2}(1+|x|)+\frac{c}{2 \sqrt{2}} \sqrt{\sum_{i=1}^{m} y_{i}^{2}}+\frac{1}{\sqrt{2}} \sqrt{\sum_{i=1}^{m} y_{i}^{2}} \sqrt{2 n c^{2} \sum_{i=1}^{m}\left|\nabla h_{i}\right|^{2}\left(\frac{x}{\sqrt{2}}\right)} \\
& +\frac{1}{\sqrt{2}} \sqrt{\sum_{i=1}^{m} y_{i}^{2}} \sqrt{2 n c^{2} \sum_{i=1}^{m} \sum_{j=1}^{n} \sum_{k=1}^{n}\left(\frac{\partial^{2} h_{i}}{\partial x_{k} \partial x_{j}}\right)^{2}\left(\frac{x}{\sqrt{2}}\right)}+\frac{n}{2 \sqrt{2}}\left(\sum_{i=1}^{m} y_{i}^{2}\right) c^{2} \\
& \leq \frac{\sqrt{n}}{2} c+\frac{c^{2}}{2}(1+|x|)+\frac{c}{2 \sqrt{2}} \sqrt{\sum_{i=1}^{m} y_{i}^{2}}+\frac{1}{\sqrt{2}} \sqrt{\sum_{i=1}^{m} y_{i}^{2} \sqrt{2 n} c^{2}}
\end{aligned}
$$

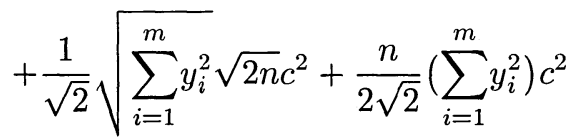

$$
\begin{aligned}
& \leq\left[\frac{\sqrt{n}}{2} c+\frac{c^{2}}{2}+\left(\frac{c}{2 \sqrt{2}}+2 \sqrt{n} c^{2}\right) \sqrt{\sum_{i=1}^{m} y_{i}^{2}}+\frac{n c^{2}}{2 \sqrt{2}}\left(\sum_{i=1}^{m} y_{i}^{2}\right)\right](1+|x|) \\
& \leq\left(\frac{\sqrt{n}}{2} c+\frac{n c^{2}}{2}\right)\left(1+\sqrt{\sum_{i=1}^{m} y_{i}^{2}}\right)^{2}(1+|x|)
\end{aligned}
$$

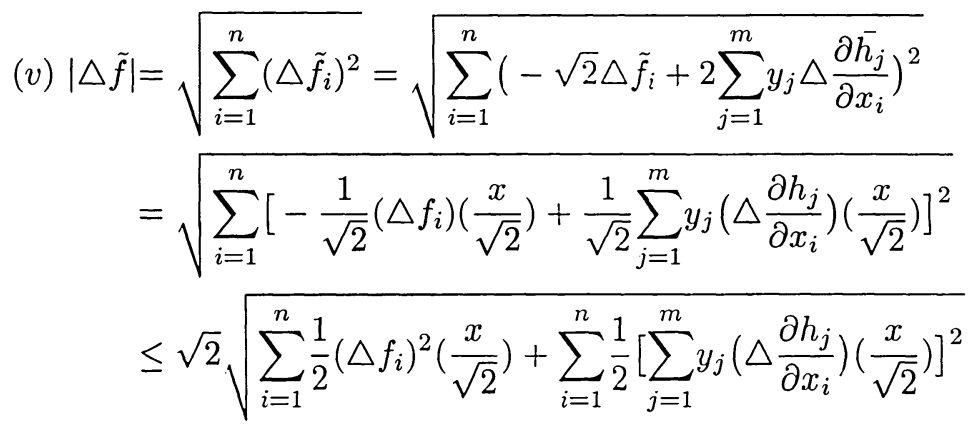




$$
\begin{aligned}
& \leq \sqrt{\sum_{i=1}^{n}\left(\Delta f_{i}\right)^{2}\left(\frac{x}{\sqrt{2}}\right)}+\sqrt{\sum_{i=1}^{n}\left[\sum_{j=1}^{m} y_{j}\left(\Delta \frac{\partial h_{j}}{\partial x_{i}}\right)\left(\frac{x}{\sqrt{2}}\right)\right]^{2}} \\
& \leq c+\sqrt{\sum_{i=1}^{n}\left(\sum_{j=1}^{m} y_{j}^{2}\right)\left(\sum_{j=1}^{m}\left(\Delta \frac{\partial h_{j}}{\partial x_{i}}\right)^{2}\left(\frac{x}{\sqrt{2}}\right)\right)} \\
& =c+\sqrt{\sum_{j=1}^{m} y_{j}^{2}} \sqrt{\sum_{i=1}^{n} \sum_{j=1}^{m}\left(\frac{\partial \Delta h_{j}}{\partial x_{i}}\right)^{2}\left(\frac{x}{\sqrt{2}}\right)} \\
& =c+\sqrt{\sum_{j=1}^{m} y_{j}^{2}} \sqrt{\sum_{j=1}^{m} \mid \nabla\left(\Delta h_{j}\right)\left(\frac{x}{\sqrt{2}}\right)} \\
& \leq\left(1+\sqrt{\left.\sum_{j=1}^{m} y_{j}^{2}\right) c}\right.
\end{aligned}
$$

(vi) $|\Delta \tilde{V}|=\mid \sqrt{2} \sum_{i=1}^{n} \triangle \bar{f}_{i, i}+\frac{1}{2} \sum_{i=1}^{m} \triangle \bar{h}_{i}^{2}-\sum_{i=1}^{m} y_{i} \triangle \triangle \bar{h}_{i}+\sqrt{2} \sum_{i=1}^{m} \sum_{j=1}^{n} y_{i} \Delta\left(\bar{f}_{j} \frac{\partial \bar{h}_{i}}{\partial x_{j}}\right)$

$$
\begin{aligned}
&-\sum_{k=1}^{n} \Delta\left[\left(\sum_{i=1}^{m} y_{i} \frac{\partial \bar{h}_{i}}{\partial x_{k}}\right)^{2}\right] \mid \\
&=\left.\left|\sqrt{2} \sum_{i=1}^{n} \Delta \bar{f}_{i, i}+\sum_{i=1}^{m} \bar{h}_{i} \Delta \bar{h}_{i}+\sum_{i=1}^{m}\right| \nabla \bar{h}_{i}\right|^{2}-\sum_{i=1}^{m} y_{i} \Delta \Delta \bar{h}_{i}+\sqrt{2} \sum_{i=1}^{m} \sum_{j=1}^{n} y_{i} \bar{f}_{j} \Delta \frac{\partial \bar{h}_{i}}{\partial x_{j}}
\end{aligned}
$$$$
+\sqrt{2} \sum_{i=1}^{m} \sum_{j=1}^{n} y_{i}\left(\triangle \bar{f}_{j}\right) \frac{\partial \bar{h}_{i}}{\partial x_{j}}+2 \sqrt{2} \sum_{i=1}^{m} \sum_{j=1}^{n} y_{i} \nabla \bar{f}_{j} \cdot \nabla \frac{\partial \bar{h}_{i}}{\partial x_{j}}
$$$$
-2 \sum_{k=1}^{n}\left(\sum_{i=1}^{m} y_{i} \frac{\partial \bar{h}_{i}}{\partial x_{k}}\right) \triangle\left(\sum_{i=1}^{m} y_{i} \frac{\partial \bar{h}_{i}}{\partial x_{k}}\right)-2 \sum_{k=1}^{n}\left|\sum_{i=1}^{m} y_{i} \nabla\left(\frac{\partial \bar{h}_{i}}{\partial x_{k}}\right)\right|^{2} \mid
$$$$
=\left.\left|\frac{1}{2} \sum_{i=1}^{n}\left(\Delta f_{i, i}\right)\left(\frac{x}{\sqrt{2}}\right)+\frac{1}{2}\left(h_{i} \Delta h_{i}\right)\left(\frac{x}{\sqrt{2}}\right)+\frac{1}{2} \sum_{i=1}^{m}\right| \nabla h_{i}\right|^{2}\left(\frac{x}{\sqrt{2}}\right)
$$$$
-\frac{1}{4} \sum_{i=1}^{m} y_{i} \triangle \triangle h_{i}+\frac{1}{2} \sum_{i=1}^{m} \sum_{j=1}^{n} y_{i}\left(f_{j} \triangle \frac{\partial h_{i}}{\partial x_{j}}\right)\left(\frac{x}{\sqrt{2}}\right)
$$$$
+\sum_{i=1}^{m} \sum_{j=1}^{n} y_{i}\left(\nabla f_{j} \cdot \nabla \frac{\partial h_{i}}{\partial x_{j}}\right)\left(\frac{x}{\sqrt{2}}\right)+\frac{1}{2} \sum_{i=1}^{m} \sum_{j=1}^{n} y_{i}\left(\triangle f_{j}\right) \frac{\partial h_{i}}{\partial x_{j}}\left(\frac{x}{\sqrt{2}}\right)
$$

$$
\begin{aligned}
& -\frac{1}{2} \sum_{k=1}^{n}\left(\sum_{i=1}^{m} y_{i} \frac{\partial h_{i}}{\partial x_{k}}\right)\left(\sum_{i=1}^{m} y_{i} \Delta \frac{\partial h_{i}}{\partial x_{k}}\right)\left(\frac{x}{\sqrt{2}}\right)-\frac{1}{2} \sum_{k=1}^{n}\left|\sum_{i=1}^{m} y_{i} \nabla\left(\frac{\partial h_{i}}{\partial x_{k}}\right)\right|^{2}\left(\frac{x}{\sqrt{2}}\right) \mid \\
\leq & \frac{1}{2} \sum_{i=1}^{n}\left|\frac{\partial \triangle f_{i}}{\partial x_{i}}\right|\left(\frac{x}{\sqrt{2}}\right)+\frac{1}{2} \sqrt{\sum_{i=1}^{m} h_{i}^{2}\left(\frac{x}{\sqrt{2}}\right)} \sqrt{\sum_{i=1}^{m}\left(\Delta h_{i}\right)^{2}\left(\frac{x}{\sqrt{2}}\right)+\frac{1}{2} \sum_{i=1}^{m}\left|\nabla h_{i}\right|^{2}\left(\frac{x}{\sqrt{2}}\right)} \\
& +\frac{1}{4} \sqrt{\sum_{i=1}^{m} y_{i}^{2}} \sqrt{\sum_{i=1}^{m}\left(\Delta \Delta h_{i}\right)^{2}\left(\frac{x}{\sqrt{2}}\right)}+\frac{1}{2} \sum_{i=1}^{m}\left|y_{i}\right| \sqrt{\sum_{j=1}^{n} f_{j}^{2}\left(\frac{x}{2}\right)} \sqrt{\sum_{j=1}^{n}\left[\Delta \frac{\partial h_{i}}{\partial x_{j}}\right]^{2}\left(\frac{x}{\sqrt{2}}\right)}
\end{aligned}
$$




$$
\begin{aligned}
& +\sum_{i=1}^{m}\left|y_{i}\right| \sqrt{\sum_{j=1}^{n}\left|\nabla f_{j}\right|^{2}\left(\frac{x}{\sqrt{2}}\right)} \sqrt{\sum_{j=1}^{n}\left|\nabla \frac{\partial h_{i}}{\partial x_{j}}\right|^{2}\left(\frac{x}{\sqrt{2}}\right)} \\
& +\frac{1}{2} \sum_{i=1}^{m}\left|y_{i}\right| \sqrt{\sum_{j=1}^{n}\left(\triangle f_{j}\right)^{2}\left(\frac{x}{\sqrt{2}}\right)} \sqrt{\sum_{j=1}^{n}\left(\frac{\partial h_{i}}{\partial x_{j}}\right)^{2}\left(\frac{x}{\sqrt{2}}\right)} \\
& +\frac{1}{2} \sum_{k=1}^{n} \sqrt{\sum_{i=1}^{m} y_{i}^{2}} \sqrt{\sum_{i=1}^{m}\left(\frac{\partial h_{i}}{\partial x_{k}}\right)^{2}\left(\frac{x}{\sqrt{2}}\right)} \sqrt{\sum_{i=1}^{m} y_{i}^{2}} \sqrt{\sum_{i=1}^{m}\left(\triangle \frac{\partial h_{i}}{\partial x_{k}}\right)^{2}\left(\frac{x}{\sqrt{2}}\right)} \\
& +\frac{1}{2} \sum_{k=1}^{n}\left(\sum_{i=1}^{m} y_{i}^{2}\right)\left(\sum_{i=1}^{m}\left|\nabla \frac{\partial h_{i}}{\partial x_{k}}\right|^{2}\left(\frac{x}{\sqrt{2}}\right)\right. \\
& \leq \frac{\sqrt{n}}{2} \sqrt{\sum_{i=1}^{n}\left(\frac{\partial \triangle f_{i}}{\partial x_{i}}\right)^{2}}+\frac{1}{2} c\left(1+\frac{|x|}{2}\right) \frac{c}{1+\frac{|x|}{2}}+\frac{c^{2}}{2} \\
& +\frac{1}{4} \sqrt{\sum_{i=1}^{m} y_{i}^{2} c}+\frac{1}{2} c\left(1+\frac{|x|}{2}\right) \sqrt{\sum_{i=1}^{m} y_{i}^{2}} \sqrt{\sum_{i=1}^{m} \sum_{j=1}^{n}\left(\frac{\partial}{\partial x_{j}} \Delta h_{i}\right)^{2}\left(\frac{x}{\sqrt{2}}\right)} \\
& +c \sqrt{\sum_{i=1}^{m} y_{i}^{2}} \sqrt{\sum_{i=1}^{m} \sum_{j=1}^{n}\left|\nabla \frac{\partial h_{i}}{\partial x_{j}}\right|^{2}\left(\frac{x}{\sqrt{2}}\right)}+\frac{c}{2} \sqrt{\sum_{i=1}^{m} y_{i}^{2}} \sqrt{\sum_{i=1}^{m} \sum_{j=1}^{n}\left(\frac{\partial h_{i}}{\partial x_{j}}\right)^{2}\left(\frac{x}{\sqrt{2}}\right)} \\
& +\frac{1}{2}\left(\sum_{i=1}^{m} y_{i}^{2}\right) \sqrt{\sum_{k=1}^{n} \sum_{i=1}^{m}\left(\frac{\partial h_{i}}{\partial x_{k}}\right)^{2}\left(\frac{x}{\sqrt{2}}\right)} \sqrt{\sum_{k=1}^{n} \sum_{i=1}^{m}\left(\triangle \frac{\partial h_{i}}{\partial x_{k}}\right)^{2}\left(\frac{x}{\sqrt{2}}\right)} \\
& +\frac{1}{2} \sum_{i=1}^{m} y_{i}^{2} \sum_{k=1}^{n} \sum_{i=1}^{m}\left|\nabla \frac{\partial h_{i}}{\partial x_{k}}\right|^{2}\left(\frac{x}{\sqrt{2}}\right) \\
& \leq \frac{\sqrt{n}}{2} \sqrt{\sum_{i=1}^{n}\left|\nabla \Delta f_{i}\right|^{2}}+\frac{c^{2}}{2}+\frac{c^{2}}{2}+\frac{c}{4} \sqrt{\sum_{i=1}^{m} y_{i}^{2}} \\
& +\frac{c}{2}\left(1+\frac{|x|}{2}\right) \sqrt{\sum_{i=1}^{m} y_{i}^{2}} \sqrt{\sum_{i=1}^{m}\left|\nabla \Delta h_{i}\right|^{2}\left(\frac{x}{\sqrt{2}}\right)} \\
& +c \sqrt{\sum_{i=1}^{m} y_{i}^{2}} \sqrt{\sum_{i=1}^{m} \sum_{j=1}^{n} \sum_{k=1}^{n}\left(\frac{\partial^{2} h_{i}}{\partial x_{j} \partial x_{k}}\right)^{2}\left(\frac{x}{\sqrt{2}}\right)} \\
& +\frac{c^{2}}{2} \sqrt{\sum_{i=1}^{m} y_{i}^{2}}+\frac{1}{2}\left(\sum_{i=1}^{m} y_{i}^{2}\right) \sqrt{\sum_{i=1}^{m}\left|\nabla h_{i}\right|^{2}\left(\frac{x}{\sqrt{2}}\right)} \sqrt{\sum_{i=1}^{m}\left|\nabla \Delta h_{i}\right|^{2}\left(\frac{x}{\sqrt{2}}\right)} \\
& +\frac{1}{2} \sum_{i=1}^{m} y_{i}^{2} \sum_{k=1}^{n} \sum_{i=1}^{m} \sum_{j=1}^{n}\left(\frac{\partial^{2} h_{i}}{\partial x_{j} \partial x_{k}}\right)^{2}\left(\frac{x}{\sqrt{2}}\right) \\
& \leq \frac{\sqrt{n}}{2} c+\frac{c^{2}}{2}+\frac{c^{2}}{2}+\frac{c}{4} \sqrt{\sum_{i=1}^{m} y_{i}^{2}}+\frac{c^{2}}{2} \sqrt{\sum_{i=1}^{m} y_{i}^{2}}+c^{2} \sqrt{\sum_{i=1}^{m} y_{i}^{2}}
\end{aligned}
$$




$$
\begin{aligned}
& +\frac{c^{2}}{2} \sqrt{\sum_{i=1}^{m} y_{i}^{2}}+\frac{c^{2}}{2}\left(\sum_{i=1}^{m} y_{i}^{2}\right)+\frac{c^{2}}{2} \sum_{i=1}^{m} y_{i}^{2} \\
= & \frac{\sqrt{n}}{2} c+c^{2}+\frac{c}{4} \sqrt{\sum_{i=1}^{m} y_{i}^{2}}+2 c^{2} \sqrt{\sum_{i=1}^{m} y_{i}^{2}}+c^{2}\left(\sum_{i=1}^{m} y_{i}^{2}\right) \\
= & \left(\frac{\sqrt{n}}{2}+c\right) c+\left(\frac{c}{4}+2 c^{2}\right) \sqrt{\sum_{i=1}^{m} y_{i}^{2}}+c^{2}\left(\sum_{i=1}^{m} y_{i}^{2}\right) \\
\leq & \left(\frac{\sqrt{n}}{2} c+c^{2}\right)\left(1+\sqrt{\left.\sum_{i=1}^{m} y_{i}^{2}\right)^{2}}\right.
\end{aligned}
$$

Remark 5.1 Under the assumptions of Proposition 5.2, we can take

$$
\bar{c}(t)=\left[\frac{\sqrt{n}}{2} c+\frac{(n+1) c^{2}}{2}\right]\left(1+\sqrt{\sum_{i=1}^{m} y_{i}^{2}}\right)^{2}
$$

so that (5.8), (5.9), (5.10) are satisfied. $\square$

In view of Proposition 3.1, we have the following Harnack inequality.

THEOREM 5.3. Let $\bar{u}_{R}>0$ be a positive solution of the equation (5.5) on the closed ball $B_{R}=\left\{x \in \mathbb{R}^{n}:|x| \leq R\right\}$ with the Neumann condition $\frac{\partial \bar{u}_{R}}{\partial \nu}=0$ on $\partial B_{R}$. Let $\bar{\varphi}(x, t)=-\log \bar{u}_{R}(x, t)$ and $\bar{\beta}(x, t)=\bar{c}_{2}(t)|x|^{2}+4 \bar{\alpha}^{2}(t)+\frac{n}{2 t}$ with $\bar{c}_{2}(t)>0$ and $\bar{\alpha}(t)>0$. Suppose that (5.8)-(5.18) hold so that

$$
\bar{\varphi}_{t}+|\nabla \bar{\varphi}|^{2}-\sum_{j=1}^{n} \tilde{f}_{j} \frac{\partial \bar{\varphi}}{\partial x_{j}}-\tilde{V}<\bar{\alpha}(t) \sqrt{|\nabla \bar{\varphi}|^{2}+\bar{\beta}(x, t)}+\frac{n}{2 t}+2 \sqrt{n} \bar{c}(t)
$$

for all $t>0$. Let $t>t_{0}$ and $\mathcal{P}=\left\{\right.$ differentiable path $\sigma=,\left(\sigma_{1}, \sigma_{2}\right):[0,1] \rightarrow$ $B_{R} \times \mathbb{R}$ such that $\left.\sigma(0)=\left(\sigma_{1}(0), \sigma_{2}(0)\right)=\left(x_{0}, t_{0}\right), \sigma(1)=(x, t), \sigma_{2}^{\prime}(s)>0\right\}$. Define

$$
\begin{aligned}
d\left(\left(x_{0}, t_{0}\right),(x, t)\right) & =\inf _{\sigma \in \mathcal{P}}\left\{\frac{1}{2} \int_{0}^{1}<\dot{\sigma}_{1}, \tilde{f}>d s+\int_{0}^{1} \frac{d \sigma_{2}}{d s}\left(\sqrt{2} \bar{\alpha} \sqrt{\frac{|\tilde{f}|^{2}}{4}+\frac{\bar{\beta}}{2}}\right.\right. \\
& \left.\left.+\tilde{V}+\frac{1}{4} \sum_{i=1}^{n} \tilde{f}_{i}^{2}\right) d s+\frac{1}{4} \int_{0}^{1} \frac{d \sigma_{2}}{d s}\left[\frac{\left|\dot{\sigma}_{1}\right|}{\frac{d \sigma_{2}}{d s}}+\sqrt{2} \bar{\alpha}\right]^{2} d s\right\}
\end{aligned}
$$

Then

$(5.21) \bar{u}_{R}(x, t) \geq \bar{u}_{R}\left(x_{0}, t_{0}\right)\left(\frac{t}{t_{0}}\right)^{-\frac{n}{2}} \exp \left(-\int_{t_{0}}^{t} 2 \sqrt{n} \bar{c}(\tau) d \tau\right) \exp \left[-d\left(\left(x_{0}, t_{0}\right),(x, t)\right)\right]$

Similarly Theorem 3.3 gives us the following Harnack inequality. 
THEOREM 5.4. Let $\bar{u}>0$ be a positive solution of the equation

$$
\bar{u}_{t}=\Delta \bar{u}+\sum_{i=1}^{n} \tilde{f}_{i} \bar{u}_{i}-\tilde{V} \bar{u}
$$

on $\mathbb{R}^{n}$. Let $\bar{\varphi}(x, t)=-\log \bar{u}(x, t)$ and $\bar{\beta}(x, t)=\bar{c}_{2}(t)|x|^{2}+4 \bar{\alpha}^{2}(t)+\frac{n}{2 t}$ with $\bar{c}_{2}(t)>$ 0 and $\bar{\alpha}(t)>0$. Suppose that

$$
\begin{aligned}
& |\tilde{f}| \leq \bar{c}(t)(1+|x|) \\
& |\nabla \tilde{f}|=\sqrt{\sum_{i=1}^{n}\left|\nabla \tilde{f}_{i}\right|^{2}} \leq \bar{c}(t),|\nabla \tilde{V}| \leq \bar{c}(1+|x|) \\
& |\triangle \tilde{V}| \leq \bar{c},|\triangle \tilde{f}| \leq \bar{c} \\
& 2 \bar{c}^{2}(t) \leq \bar{c}_{1}(t) \bar{c}_{2}(t) \\
& 2 \bar{c}^{2}(t) \leq \bar{c}_{1}(t)\left(4 \bar{\alpha}^{2}(t)+\frac{n}{2 t}\right) \\
& 4 \bar{c}_{2}(t) \leq \bar{c}_{1}^{2}(t) \\
& \bar{c}_{2}^{\prime}+\frac{3}{t} \bar{c}_{2} \geq 0 \\
& 2\left(\bar{\alpha}^{2}\right)^{\prime}+\frac{6}{t} \bar{\alpha}^{2}+\frac{n}{4 t^{2}}>0 \\
& \bar{\alpha}^{\prime}(t)+\bar{\alpha}\left[\frac{3 \bar{c}}{2 \sqrt{n}}-\bar{\lambda}-\frac{1}{2}\left(\sqrt{\bar{c}_{1}}+\bar{c}_{1}+\bar{c}_{1}^{3 / 2}\right)\right]-\sqrt{n} \bar{c} \geq 0 \\
& \bar{c}^{\prime}(t)+\frac{5}{48 \sqrt{n}} \bar{c}^{+}\left(\frac{3}{8 t}-\frac{1}{2 \sqrt{n}}\right) \bar{c}-\frac{\sqrt{n}}{2} \bar{c}_{2}>0
\end{aligned}
$$

where $\bar{\lambda}$ is the absolute value of the greatest eigenvalue of $\left(\frac{\tilde{f}_{i . j}+\tilde{f}_{j, i}}{2}\right)$. Let

$$
\begin{aligned}
\bar{\Psi}(x, t) & =\bar{\varphi}_{t}+|\nabla \varphi|^{2}-\sum_{j=1}^{n} \tilde{f}_{j} \frac{\partial \bar{\varphi}}{\partial x_{j}}-\tilde{V}-\bar{\alpha}(t) \sqrt{|\nabla \varphi|^{2}+\bar{\beta}(x, t)}-\frac{n}{2 t}-2 \sqrt{n} \bar{c}(t) \\
& =\triangle \varphi-\bar{\alpha}(t) \sqrt{|\nabla \varphi|^{2}+\bar{\beta}(x, t)}-\frac{n}{2 t}-2 \sqrt{n} \bar{c}(t)
\end{aligned}
$$

Let $t>t_{0}$ and $\mathcal{P}=\left\{\right.$ diferentiable path $\sigma=\left(\sigma_{1}, \sigma_{2}\right):[0,1] \rightarrow \mathbb{R}^{n} \times \mathbb{R}$ such that $\sigma(0)$ $\left.=\left(\sigma_{1}(0), \sigma_{2}(0)\right)=\left(x_{0}, t_{0}\right), \sigma(1)=(x, t), \sigma_{2}^{\prime}(s)>0\right\}$. Define

$$
\begin{aligned}
& d\left(\left(x_{0}, t_{0}\right),(x, t)\right)=\inf _{\sigma \in \mathcal{P}}\{ \frac{1}{2} \int_{0}^{1}<\dot{\sigma}_{1}, \tilde{f}>d s+\int_{0}^{1} \frac{d \sigma_{2}}{d s}\left(\sqrt{2} \bar{\alpha} \sqrt{\frac{|\tilde{f}|^{2}}{4}+\frac{\bar{\beta}}{2}}\right. \\
&\left.\left.+\tilde{V}+\frac{1}{4} \sum_{i=1}^{n} \tilde{f}_{i}^{2}\right) d s+\frac{1}{4} \int_{0}^{1} \frac{d \sigma_{2}}{d s}\left[\frac{\left|\dot{\sigma}_{1}\right|}{\frac{d \sigma_{2}}{d s}}+\sqrt{2} \bar{\alpha}\right]^{2} d s\right\}
\end{aligned}
$$

If $\Psi(x, 0)<0$, then

(5.33) $\bar{u}(x, t) \geq \bar{u}\left(x_{0}, t_{0}\right)\left(\frac{t}{t_{0}}\right)^{-\frac{n}{2}} \exp \left(-\int_{t_{0}}^{t} 2 \sqrt{n} \bar{c}(\tau) d \tau\right) \exp \left[-d\left(\left(x_{0}, t_{0}\right),(x, t)\right)\right]$ 
It is a very interesting question to find path $\sigma$ that maximize the right hand side of (5.33). It is likely that such a path carries most information of how probability density propagates in time.

In order to apply (5.33) for proving decay of the solution $\bar{u}$, we need some integral estimate of $\bar{u}$.

THEOREM 5.5. Let $\bar{u}$ be a nonnegatie solution of the equation

$$
\frac{\partial \bar{u}}{\partial t}=\Delta \bar{u}+\sum_{i=1}^{n} \tilde{f}_{i} \frac{\partial \bar{u}}{\partial x_{i}}-\tilde{V} \bar{u}
$$

on $\mathbb{R}^{n}$, where $\tilde{f}$ and $\tilde{V}$ are given by (5.6) and (5.7). Suppose that

$$
\begin{aligned}
& |f| \leq c(t)(1+|x|),|\nabla f|=\sqrt{\sum_{i=1}^{n}\left|\nabla f_{i}\right|^{2}} \leq c(t)(1+|x|) \\
& |h| \leq c(t)\left(1+|x|^{2}\right),|\nabla h|=\sqrt{\sum_{i=1}^{m}\left|\nabla h_{i}\right|^{2}} \leq c(t)
\end{aligned}
$$

Let $\varepsilon_{2}$ and $T$ be small enough so that for $0 \leq t \leq T$,

$$
\begin{aligned}
\frac{|x||\bar{f}| \sqrt{2}}{4\left(t+\varepsilon_{2}\right)} & \leq \frac{3 n}{4\left(t+\varepsilon_{2}\right)}+\frac{|x|^{2}}{64\left(t+\varepsilon_{2}\right)^{2}}-\frac{3}{2}\left(\sum_{j=1}^{m}\left|\nabla \bar{h}_{j}\right|^{2}\right)^{2} \\
& -\frac{1}{2} \sum_{j=1}^{m}\left(\triangle \bar{h}_{j}\right)^{2}+\frac{1}{2} \sum_{i=1}^{m} \bar{h}_{i}^{2}-\frac{\sqrt{2}}{2} \sum_{i=1}^{m}\left(\sum_{j=1}^{n} \bar{f}_{j} \frac{\partial \bar{h}_{i}}{\partial x_{j}}\right)
\end{aligned}
$$

Then

$$
\begin{aligned}
\int_{\mathbb{R}^{n}} \rho(x, t) \bar{u}(x, t) \leq & \left\{\exp \left[\frac{3}{2} \int_{0}^{T}\left(\sum_{j=1}^{m} y_{j}^{2}\right)^{2}+\frac{1+\sqrt{2}}{2} \int_{0}^{T} \sum_{j=0}^{m} y_{j}^{2}\right]\right\} \\
& \int_{\mathbb{R}^{n}} \rho(x, 0) \bar{u}(x, 0)
\end{aligned}
$$

where $\rho(x, t)=\left(t+\varepsilon_{2}\right)^{-n} \exp \left(\frac{|x|^{2}}{8\left(t+\varepsilon_{2}\right)}\right)$

Proof. Let $\rho$ be any smooth function on $\mathbb{R}^{n}$. We have

$$
\begin{aligned}
& \frac{d}{d t} \int_{B_{R}} \rho \bar{u}=\int_{B_{R}} \rho_{t} \bar{u}+\int_{B_{R}} \rho \bar{u}_{t} \\
= & \int_{B_{R}} \rho_{t} \bar{u}+\int_{B_{R}} \rho \triangle \bar{u}+\int_{B_{R}} \rho \sum_{i=1}^{n} \tilde{f}_{i} \frac{\partial \bar{u}}{\partial x_{i}}-\int_{B_{R}} \rho \tilde{V} \bar{u} \\
= & \int_{B_{R}} \rho_{t} \bar{u}+\int_{B_{R}}(\Delta \rho) \bar{u}-\int_{\partial B_{R}} \bar{u} \frac{\partial \rho}{\partial \nu} d s+\int_{\partial B_{R}} \rho \frac{\partial \bar{u}}{\partial \nu} d s \\
& -\int_{B_{R}}\left(\rho \sum_{i=1}^{n} \tilde{f}_{i, i,}+\sum_{i=1}^{n} \rho_{i} \tilde{f}_{i}\right) \bar{u}+\int_{\partial B_{R}} \bar{u} \rho \tilde{f} \cdot \nu d s-\int_{B_{R}} \rho \tilde{V} \bar{u}
\end{aligned}
$$


by Divergence Theorem and Green's first identity. Now we can use simple cut off argument to obtain the following

$$
\begin{aligned}
\frac{d}{d t} \int_{\mathbb{R}^{n}} \rho \bar{u}= & \int_{\mathbb{R}^{n}}\left(\rho_{t}+\Delta \rho-\sum_{i=1}^{n} \tilde{f}_{i} \rho_{i}-\sum_{i=1}^{n} \tilde{f}_{i, i} \rho-\tilde{V} \rho\right) \bar{u} \\
\rho= & \left(t+\varepsilon_{2}\right)^{-n} \exp \left[\frac{|x|^{2}}{8\left(t+\varepsilon_{2}\right)}\right] \\
\rho_{i}= & \frac{1}{4\left(t+\varepsilon_{2}\right)}\left(t+\varepsilon_{2}\right)^{-n} x_{i} \exp \left[\frac{|x|^{2}}{8\left(t+\varepsilon_{2}\right)}\right]=\frac{x_{i} \rho}{4\left(t+\varepsilon_{2}\right)} \\
\rho_{i i}= & \frac{\rho}{4\left(t+\varepsilon_{2}\right)}+\frac{x_{i}^{2} \rho}{16\left(t+\varepsilon_{2}\right)^{2}} \\
\triangle \rho= & {\left[\frac{n}{4\left(t+\varepsilon_{2}\right)}+\frac{|x|^{2}}{16\left(t+\varepsilon_{2}\right)^{2}}\right] \rho } \\
\rho_{t}+\triangle \rho= & -n\left(t+\varepsilon_{2}\right)^{-n-1} \exp \left[\frac{|x|^{2}}{8\left(t+\varepsilon_{2}\right)}\right]-\frac{|x|^{2}}{8\left(t+\varepsilon_{2}\right)}\left(t+\varepsilon_{2}\right)^{-n} \exp \left[\frac{|x|^{2}}{8\left(t+\varepsilon_{2}\right)}\right] \\
& +\left[\frac{n}{4\left(t+\varepsilon_{2}\right)}+\frac{|x|^{2}}{16\left(t+\varepsilon_{2}\right)^{2}}\right] \rho \\
= & {\left[\frac{-n}{t+\varepsilon_{2}}-\frac{|x|^{2}}{8\left(t+\varepsilon_{2}\right)^{2}}+\frac{n}{4\left(t+\varepsilon_{2}\right)}+\frac{|x|^{2}}{16\left(t+\varepsilon_{2}\right)^{2}}\right] \rho } \\
= & -\left[\frac{3 n}{4\left(t+\varepsilon_{2}\right)}+\frac{|x|^{2}}{16\left(t+\varepsilon_{2}\right)^{2}}\right] \rho
\end{aligned}
$$

Observe that

$$
\begin{aligned}
-\sum_{i=1}^{n} \tilde{f}_{i} \rho_{i} & \leq\left|\sum_{i=1}^{n} \tilde{f}_{i} \rho_{i}\right| \\
& =\left|\sum_{i=1}^{n} \sqrt{2} \bar{f}_{i} x_{i}-2 \sum_{i=1}^{n} \sum_{j=1}^{m} x_{i} y_{i} \frac{\partial \bar{h}_{j}}{\partial x_{i}}\right| \frac{\rho}{4\left(t+\varepsilon_{2}\right)} \\
& \leq \frac{\sqrt{2} \rho}{4\left(t+\varepsilon_{2}\right)}|x||\bar{f}|+\frac{\rho}{2\left(t+\varepsilon_{2}\right)}\left[\frac{|x|^{2}}{16\left(t+\varepsilon_{2}\right)}+4\left(t+\varepsilon_{2}\right) \sum_{i=1}^{n}\left(\sum_{j=1}^{m} y_{j} \frac{\partial \bar{h}_{j}}{\partial x_{i}}\right)^{2}\right] \\
& =\left[\frac{|x||\bar{f}| \sqrt{2}}{4\left(t+\varepsilon_{2}\right)}+\frac{|x|^{2}}{32\left(t+\varepsilon_{2}\right)^{2}}+2 \sum_{i=1}^{n}\left(\sum_{j=1}^{m} y_{j} \frac{\partial \bar{h}_{j}}{\partial x_{i}}\right)^{2}\right] \rho \\
-\left(\sum_{i=1}^{n} \tilde{f}_{i, i}+\tilde{V}\right) \rho=-\left(\sum_{i=1}^{n} \tilde{f}_{i, i}+\tilde{V}^{2} \rho\right. & -\left[\sum_{i=1}^{n}\left(-\sqrt{2} \bar{f}_{i}+2 \sum_{j=1}^{m} y_{j} \frac{\partial \bar{h}_{j}}{\partial x_{i}}\right)_{i}+\sqrt{2} \sum_{i=1}^{n} \bar{f}_{i, i}+\frac{1}{2} \sum_{i=1}^{m} \bar{h}_{i}^{2}\right. \\
& \quad-\sum_{i=1}^{m} y_{i} \triangle \bar{h}_{i}+\sqrt{2} \sum_{i=1}^{m} \sum_{j=1}^{n} y_{i} \bar{f}_{j} \frac{\partial \bar{h}_{i}}{\partial x_{j}}
\end{aligned}
$$




$$
\begin{aligned}
& \left.-\sum_{i=1}^{m} \sum_{j=1}^{m} \sum_{k=1}^{n} y_{i} y_{j} \frac{\partial \bar{h}_{i}}{\partial x_{k}} \frac{\partial \bar{h}_{j}}{\partial x_{k}}\right] \rho \\
= & -\left[-\sqrt{2} \sum_{i=1}^{n} \bar{f}_{i, i}+2 \sum_{j=1}^{m} y_{j} \Delta \bar{h}_{j}+\sqrt{2} \sum_{i=1}^{n} \bar{f}_{i, i}+\frac{1}{2} \sum_{i=1}^{m} \bar{h}_{i}^{2}\right. \\
- & \left.\sum_{i=1}^{m} y_{i} \triangle \bar{h}_{i}+\sqrt{2} \sum_{i=1}^{m} \sum_{j=1}^{n} y_{i} \bar{f}_{j} \frac{\partial \bar{h}_{i}}{\partial x_{j}}-\sum_{i=1}^{m} \sum_{j=1}^{m} \sum_{k=1}^{n} y_{i} y_{j} \frac{\partial \bar{h}_{i}}{\partial x_{k}} \frac{\partial \bar{h}_{j}}{\partial x_{k}}\right] \rho \\
=- & {\left[2 \sum_{j=1}^{m} y_{j} \triangle \bar{h}_{j}+\frac{1}{2} \sum_{i=1}^{m} \bar{h}_{i}^{2}+\sqrt{2} \sum_{i=1}^{m} \sum_{j=1}^{n} y_{i} \bar{f}_{j} \frac{\partial \bar{h}_{i}}{\partial x_{j}}\right.} \\
& \left.-\sum_{i=1}^{m} \sum_{j=1}^{m} \sum_{k=1}^{n} y_{i} y_{j} \frac{\partial \bar{h}_{i}}{\partial x_{k}} \frac{\partial \bar{h}_{j}}{\partial x_{k}}\right] \rho
\end{aligned}
$$

Hence

$$
\begin{aligned}
\frac{d}{d t} \int_{\mathbb{R}^{n}} \rho \bar{u} & \leq \int_{\mathbb{R}^{n}} \rho\left[\frac{-3 n}{4\left(t+\varepsilon_{2}\right)}-\frac{|x|^{2}}{16\left(t+\varepsilon_{2}\right)^{2}}+\frac{|x||\bar{f}| \sqrt{2}}{4\left(t+\varepsilon_{2}\right)}\right. \\
& +\frac{|x|^{2}}{32\left(t+\varepsilon_{2}\right)^{2}}+2 \sum_{i=1}^{n}\left(\sum_{j=1}^{m} y_{j} \frac{\partial \bar{h}_{j}}{\partial x_{i}}\right)^{2}-\sum_{j=1}^{m} y_{j} \Delta \bar{h}_{j}-\frac{1}{2} \sum_{i=1}^{m} \bar{h}_{i}^{2} \\
& \left.-\sqrt{2} \sum_{i=1}^{m} \sum_{j=1}^{n} y_{i} \bar{f}_{j} \frac{\partial \bar{h}_{i}}{\partial x_{j}}+\sum_{i=1}^{m} \sum_{j=1}^{m} \sum_{k=1}^{n} y_{i} y_{j} \frac{\partial \bar{h}_{i}}{\partial x_{k}} \frac{\partial \bar{h}_{j}}{\partial x_{k}}\right] \bar{u} \\
& =\int_{\mathbb{R}^{n}} \rho\left[\frac{-3 n}{4\left(t+\varepsilon_{2}\right)}-\frac{|x|^{2}}{32\left(t+\varepsilon_{2}\right)^{2}}+\frac{|x||\bar{f}| \sqrt{2}}{4\left(t+\varepsilon_{2}\right)}\right. \\
& +3 \sum_{i=1}^{n}\left(\sum_{j=1}^{m} y_{j} \frac{\partial \bar{h}_{j}}{\partial x_{i}}\right)^{2}-\sum_{j=1}^{m} y_{j} \Delta \bar{h}_{j}-\frac{1}{2} \sum_{i=1}^{m} \bar{h}_{i}^{2} \\
& \left.-\sqrt{2} \sum_{i=1}^{m} \sum_{j=1}^{n} y_{i} \bar{f}_{j} \frac{\partial \bar{h}_{i}}{\partial x_{j}}\right] \bar{u}
\end{aligned}
$$

By Schwartz inequality, we have

$$
\begin{aligned}
\sum_{i=1}^{n}\left(\sum_{j=1}^{m} y_{j} \frac{\partial \bar{h}_{j}}{\partial x_{i}}\right)^{2} & \leq \sum_{i=1}^{n}\left(\sum_{j=1}^{m} y_{j}^{2}\right)\left(\sum_{j=1}^{m} \frac{\partial \bar{h}_{j}}{\partial x_{i}}\right)^{2}=\left(\sum_{j=1}^{m} y_{j}^{2}\right)\left(\sum_{j=1}^{m}\left|\nabla \bar{h}_{j}\right|^{2}\right) \\
& \leq \frac{1}{2}\left(\sum_{j=1}^{m} y_{j}^{2}\right)^{2}+\frac{1}{2}\left(\sum_{j=1}^{m}\left|\nabla \bar{h}_{j}\right|^{2}\right)^{2} \\
\sum_{j=1}^{m} y_{j} \triangle \bar{h}_{j} & \leq\left|\sum_{j=1}^{m} y_{j} \triangle \bar{h}_{j}\right| \leq \frac{1}{2} \sum_{j=1}^{m} y_{j}^{2}+\frac{1}{2} \sum_{j=1}^{m}\left(\triangle \bar{h}_{j}\right)^{2} \\
-\sqrt{2} \sum_{i=1}^{m} \sum_{j=1}^{n} y_{i} \bar{f}_{j} \frac{\partial \bar{h}_{i}}{\partial x_{j}} & \leq \sqrt{2}\left|\sum_{i=1}^{m} \sum_{j=1}^{n}\left(\bar{f}_{j} \frac{\partial \bar{h}_{i}}{\partial x_{j}}\right) y_{i}\right| \\
& \leq \frac{\sqrt{2}}{2} \sum_{i=1}^{m}\left(\sum_{j=1}^{n} \bar{f}_{j} \frac{\partial \bar{h}_{i}}{\partial x_{j}}\right)^{2}+\frac{\sqrt{2}}{2} \sum_{i=1}^{m} y_{i}^{2}
\end{aligned}
$$


Hence

$$
\begin{aligned}
\frac{d}{d t} \int_{\mathbb{R}^{n}} \rho \bar{u} & \leq \int_{\mathbb{R}^{n}} \rho\left[\frac{-3 n}{4\left(t+\varepsilon_{2}\right)}-\frac{|x|^{2}}{32\left(t+\varepsilon_{2}\right)^{2}}+\frac{\sqrt{2}|x||\bar{f}|}{4\left(t+\varepsilon_{2}\right)}\right. \\
& +\frac{3}{2}\left(\sum_{j=1}^{m} y_{j}^{2}\right)^{2}+\frac{3}{2}\left(\sum_{j=1}^{m}\left|\nabla \bar{h}_{j}\right|^{2}\right)^{2}+\frac{1}{2} \sum_{j=1}^{m} y_{j}^{2}+\frac{1}{2} \sum_{j=1}^{m}\left(\triangle \bar{h}_{j}\right)^{2} \\
& \left.-\frac{1}{2} \sum_{i=1}^{m} \bar{h}_{i}^{2}+\frac{\sqrt{2}}{2} \sum_{i=1}^{m}\left(\sum_{j=1}^{m} \bar{f}_{j} \frac{\partial \bar{h}_{i}}{\partial x_{j}}\right)^{2}+\frac{\sqrt{2}}{2} \sum_{i=1}^{m} y_{i}^{2}\right] \bar{u} \\
& =\int_{\mathbb{R}^{n}} \rho\left[\frac{-3 n}{4(t+\varepsilon)}-\frac{|x|^{2}}{32\left(t+\varepsilon_{2}\right)^{2}}+\frac{\sqrt{2}|x||f|}{4\left(t+\varepsilon_{2}\right)}+\frac{3}{2}\left(\sum_{i=1}^{m} y_{j}^{2}\right)^{2}\right. \\
+ & \frac{3}{2}\left(\sum_{j=1}^{m}\left|\nabla \bar{h}_{j}\right|^{2}\right)^{2}+\frac{1+\sqrt{2}}{2} \sum_{j=1}^{m} y_{j}^{2}+\frac{1}{2} \sum_{j=1}^{m}\left(\triangle \bar{h}_{j}\right)^{2}-\frac{1}{2} \sum_{i=1}^{m} \bar{h}_{i}^{2} \\
+ & \left.\frac{\sqrt{2}}{2} \sum_{i=1}^{m}\left(\sum_{j=1}^{n} \bar{f}_{j} \frac{\partial \bar{h}_{i}}{\partial x_{j}}\right)^{2}\right] \bar{u}
\end{aligned}
$$

Choose $\varepsilon_{2}$ and $T$ small enough so that for $0 \leq t \leq T$, we have

$$
\begin{aligned}
\frac{|x||f| \sqrt{2}}{4\left(t+\varepsilon_{2}\right)} & \leq \frac{3 n}{4\left(t+\varepsilon_{2}\right)}+\frac{|x|^{2}}{64\left(t+\varepsilon_{2}\right)^{2}}-\frac{3}{2}\left(\sum_{j=1}^{m}\left|\nabla \bar{h}_{j}\right|^{2}\right)^{2}-\frac{1}{2} \sum_{j=1}^{m}\left(\triangle \bar{h}_{j}\right)^{2} \\
& +\frac{1}{2} \sum_{i=1}^{m} \bar{h}_{i}^{2}-\frac{\sqrt{2}}{2} \sum_{i=1}^{m}\left(\sum_{j=1}^{n} \bar{f}_{j} \frac{\partial \bar{h}_{i}}{\partial x_{j}}\right)^{2}
\end{aligned}
$$

Then we have

$$
\frac{d}{d t} \int_{\mathbb{R}^{n}} \rho \bar{u} \leq\left[\frac{3}{2}\left(\sum_{j=1}^{m} y_{j}^{2}\right)^{2}+\frac{1+\sqrt{2}}{2} \sum_{j=1}^{m} y_{j}^{2}\right] \int_{\mathbb{R}^{n}} \rho \bar{u}
$$

This implies

$$
\int_{\mathbb{R}^{n} \times\{t\}} \rho \bar{u} \leq\left\{\exp \left[\frac{3}{2} \int_{0}^{T}\left(\sum_{j=1}^{m} y_{j}^{2}\right)^{2}+\frac{1+\sqrt{2}}{2} \int_{0}^{T} \sum_{j=1}^{m} y_{j}^{2}\right]\right\} \int_{\mathbb{R}^{n} \times\{0\}} \rho \bar{u}
$$

口

Now we are ready to do the pointwise estimate.

TheOREM 5.6. Let $\bar{u}$ be a nonnegative solution of the equation

$$
\frac{\partial \bar{u}}{\partial t}=\Delta \bar{u}+\sum_{i=1}^{n} \tilde{f}_{i} \frac{\partial \bar{u}}{\partial x_{i}}-\tilde{V} \bar{u}
$$

Suppose that the assumptions of Proposition 5.2 hold. Suppose further that (5.22)(5.31) hold. Let $\varepsilon_{2}$ and $2 T$ be chosen small enough so that for $0 \leq t \leq 2 T$, (5.35) holds. Then for $x \in B_{R / 2}$. 


$$
\begin{aligned}
\bar{u}(x, t) \leq & \frac{1}{\omega_{n}}\left[\frac{R}{2\left(2 t+\varepsilon_{2}\right)}\right]^{-n}\left[\exp \left(\frac{-\left(\frac{R}{2}-|x|\right)^{2}}{8\left(2 t+\varepsilon_{2}\right)}\right)\right] \\
& (2)^{\frac{n}{2}}\left[\exp \int_{t}^{2 t} 2 \sqrt{n} \bar{c}(s) d s\right] \max _{|z-x| \leq R / 2} \exp [d((x, t),(z, 2 t))] \\
& \left\{\exp \left[\frac{3}{2} \int_{0}^{2 t}\left(\sum_{j=1}^{m} y_{j}^{2}\right)^{2}+\frac{1+\sqrt{2}}{2} \int_{0}^{2 t} \sum_{j=1}^{m} y_{j}^{2}\right]\right\} \int_{\mathbb{R}^{n}} \rho(x, 0) \bar{u}(x, 0)
\end{aligned}
$$

where $\rho(x, t)=\left(t+\varepsilon_{2}\right)^{-n} \exp \left(\frac{|x|^{2}}{8\left(t+\varepsilon_{2}\right)}\right)$ and $\omega_{n}=$ volume of unit ball.

Proof. For $x \in B_{R / 2}$ and $z \in B_{R}$. Theorem 5.4 implies

$$
\bar{u}(z, 2 t) \geq \bar{u}(x, t)\left(\frac{t}{2 t}\right)^{n / 2}\left[\exp \left(-\int_{t}^{2 t} 2 \sqrt{n} \bar{c}(s) d s\right)\right] \exp [-d((x, t),(z, 2 t))]
$$

which is equivalent to

$$
\bar{u}(x, t) \leq \bar{u}(z, 2 t) 2^{n / 2}\left[\exp \int_{t}^{2 t} 2 n \bar{c}(s) d s\right] \exp [d((x, t),(z, 2 t))]
$$

Multiplying both sides of (5.38) by $\rho(z, 2 t)$ and integrating over a closed ball with center $x$ and radius $R / 2$, we get

$$
\begin{aligned}
& \bar{u}(x, t) \int_{|z-x| \leq R / 2} \rho(z, 2 t) d z \leq 2^{n / 2}\left[\exp \int_{t}^{2 t} 2 \sqrt{n} \bar{c}(s) d s\right] \\
& \int_{|z-x| \leq R / 2} \bar{u}(z, 2 t) \rho(z, 2 t) \exp [d((x, t),(z, 2 t))] d z \\
& \leq 2^{n / 2}\left[\exp \int_{t}^{2 t} 2 \sqrt{n} \bar{c}(s) d s\right] \max _{|z-x| \leq R / 2} d((x, t),(z, 2 t)) \int_{\mathbb{R}^{n} \times\{2 t\}} \bar{u} \rho \\
& \leq 2^{n / 2}\left[\exp \int_{t}^{2 t} 2 \sqrt{n} \bar{c}(s) d s\right] \max _{|z-x| \leq R / 2} d((x, t),(z, 2 t)) \\
&\left\{\exp \left[\frac{3}{2} \int_{t}^{2 t}\left(\sum_{j=1}^{m} y_{j}^{2}\right)^{2}+\frac{1+\sqrt{2}}{2} \int_{0}^{2 t} \sum_{j=1}^{m} y_{j}^{2}\right]\right\} \int_{\mathbb{R}^{n}} \rho(x, 0) \bar{u}(x, 0)
\end{aligned}
$$

by Theorem 5.5 . Let $z=x+\frac{R}{2} z_{1}$. Then

$$
\begin{aligned}
\int_{|z-x| \leq R / 2} \rho(z, 2 t) d z & =\int_{|z-x| \leq R / 2} \frac{1}{\left(2 t+\varepsilon_{2}\right)^{n}} \exp \left[\frac{|z|^{2}}{8\left(2 t+\varepsilon_{2}\right)}\right] d z \\
& =\int_{\left|z_{1}\right| \leq 1} \frac{\left(\frac{R}{2}\right)^{n}}{\left(2 t+\varepsilon_{2}\right)^{n}} \exp \left[\frac{|x|+\left.\frac{R}{2} z_{1}\right|^{2}}{8\left(2 t+\varepsilon_{2}\right)}\right] d z_{1} \\
& \geq \omega_{n}\left[\frac{R}{2\left(2 t+\varepsilon_{2}\right)}\right]^{n} \exp \left[\frac{\left(|x|-\frac{R}{2}\right)^{2}}{8\left(2 t+\varepsilon_{2}\right)}\right]
\end{aligned}
$$

Combining (5.39) and (5.40), we get 


$$
\begin{aligned}
& \bar{u}(x, t) \omega_{n}\left[\frac{R}{2\left(2 t+\varepsilon_{2}\right)}\right]^{n} \exp \left(\frac{\left(|x|-\frac{R}{2}\right)^{2}}{8\left(2 t+\varepsilon_{2}\right)}\right) \\
\leq & \bar{u}(x, t) \int_{|z-x| \leq R / 2} \rho(z, 2 t) d z \\
\leq & 2^{\frac{n}{2}}\left[\exp \int_{t}^{2 t} 2 \sqrt{n} \bar{c}(s) d s\right]\left\{\max _{|z-x| \leq R / 2} \exp [d((x, t),(z, 2 t))]\right\} \\
& \quad\left\{\exp \left[\frac{3}{2} \int_{0}^{2 t}\left(\sum_{j=1}^{m} y_{j}^{2}\right)^{2}+\frac{1+\sqrt{2}}{2} \int_{0}^{2 t} \sum_{j=1}^{m} y_{j}^{2}\right]\right\} \int_{\mathbb{R}^{n}} \rho(x, 0) \vec{u}(x, 0)
\end{aligned}
$$

(5.37) follows immediately from (5.41).

\section{REFERENCES}

[1] J. BARAs, G. L. Blankenship, AND W. Hopkins, Existence, uniqueness, and asymptotic behavior of solutions to a class of Zakai equations with unbounded coefficients, IEEE Transactions on Automatic Control, AC-28:2 (1983), pp. 203-214.

[2] P. BESALA, Fundamental solution and Cauchy problem for a parabolic system with unbounded coefficients, J. Diff. Eq., 33 (1979), pp. 26-38.

[3] P. BESALA, On the existence of a fundamental solution for a parabolic differential equation with unbounded coefficients, Annals Polonici Mathematici, XXIX (1975), pp. 403-409

[4] W. Fleming AND S. MitTer, Optimal control and nonlinear filtering for nondegenerate diffusion processes, Stochestics, 8 (1982), pp. 63-77.

[5] A. Friedman, Partial Differential Equations of Parabolic Type, Prentice-Hall Inc., Englewood Cliffs, N.J., 1964.

[6] Z. LIANG, S. S. T. YAU, AND S. T. YAU, Finite dimensional filter with nonlinear drift $V$ : Solution to Kolmogorov equation arising from linear filtering with non-Gaussian initial condition, IEEE Transactions on Aerospace and Electronic Systems, 33:4 (1997), pp. 12951308.

[7] H. Sussmann, Rigorous results on the cubic sensor problem, stochastic system: The mathematics of filtering and identification and applications, Nato Advanced Study Institute Series, Reidal, 1981, pp. 637-648.

[8] S. T. YAU, On the Harnack inequalities of partial differential equations, Communications in Analysis and Geometry, 2:3 (1994), pp. 431-450.

[9] S. T. YAU, Harnack inequality for non-self-adjoint evolution equations, Math. Research Letter, 2 (1995), pp. 387-399.

[10] S. S. T. YAU AND S. T. YAU, Explicit formal solution to generalized Kolmogorov equation, Eleventh Army Conference on Applied Mathematics and Computing, ARO Rpt 94-1, 1994, pp. 373-386.

[11] S. T. YAU AND S. S. T. YAU, Explicit solution of a Kolmogorov equation, Applied Mathematics and Optimization, An International Journal, 34 (1996), pp. 231-266.

[12] S. T. YAU AND S. S. T. YAU, Finite dimensional filters with nonlinear drift, XI: Explicit solution of the generalized Kolmogorov equation in Brockett-Mitter Program, Advances in Mathematics, 140 (1998), pp. 156-189. 
